\title{
Ionically-Coupled Non-Linear Variable Resistors for Neuromorphic Applications
}

\author{
by \\ Aaron Michael Lee, B.Eng. \\ A thesis submitted to the Faculty of Graduate and Postdoctoral Affairs \\ in partial fulfillment of the requirements for the degree of \\ Master of Applied Science \\ in \\ Electrical and Computer Engineering \\ Ottawa-Carleton Institute for Electrical and Computer Engineering \\ Department of Electronics \\ Carleton University \\ Ottawa, Ontario \\ June, 2016
}

Copyright (C) 2016

Aaron Michael Lee 


\section{Abstract}

A means of simulating and fabricating memristor-like non-linear resistors for use in large arrays to form cellular neural networks is developed and investigated. The device is based on the action of ion intercalation of lithium ions into PEDOT:PSS active layers causing resistance to increase, similar to a memristor. A simple model is developed and implemented in SPICE to predict device behaviour, and a fabrication technique to rapidly create such a device by hand is detailed. Device testing confirmed the non-self-crossing hysteresis behaviour at a frequency of $10 \mathrm{~Hz}$ given a sinusoidal input. These results are compared to the aforementioned simulations as well as previous measurements performed in the group on similar devices, and are shown to be reasonably accurate. 


\section{Acknowledgments}

I would like to thank my supervisor, Steve McGarry, for his help finding next steps when I got stuck and for being patient when progress was slow. I would like to also thank the people in graduate office and the DOE administrative staff (Blazenka, Anna, and Sylvie) for giving me the chance to see this work through to completion.

I also want to thank my family for always supporting my efforts and endlessly encouraging me, and my friends at school for letting me think out loud at them and for constantly helping me to figure things out. In particular, I would like to thank Tom Pepler for always being willing to help me understand something I was struggling with, and Stephen Toth for his help in encouraging me and proof-reading this thesis.

I would also like to thank Prof. N. Garry Tarr, Rob Vandusen, Nagui Mikhail, and Sean Follows for their help finding and using equipment in the labs.

Finally I would like to thank anyone else who helped me, either directly or indirectly, by letting me discuss things with them. 


\section{Table of Contents}

$\begin{array}{lll}\text { Abstract } & \text { ii }\end{array}$

Acknowledgments

Table of Contents $\quad$ iv

List of Tables $\quad$ vii

List of Figures $\quad$ viii

Abbreviations and Symbols $\quad$ xiv

1 Introduction 1

1.1 Objectives . . . . . . . . . . . . . . . . . . 1

1.2 Outline. . . . . . . . . . . . . . . . . . . 1

2 Background 3

2.1 Cellular Neural Networks . . . . . . . . . . . . . . . . . . . . . . 3

2.1.1 Applications of Cellular Neural Networks . . . . . . . . . . 5

2.2 Non-Linear Resistive Elements . . . . . . . . . . . . . . . . . . 5

2.2 .1 Memristors . . . . . . . . . . . . . . 6

2.2 .2 The First Memristors . . . . . . . . . . . . . . . 8

2.2 .3 Memristor-like Devices . . . . . . . . . . . . . . . . . . 9 9 
2.3 Conductive Polymers . . . . . . . . . . . . . . . . . . . . . . 10

$2.3 .1 \quad$ PEDOT:PSS . . . . . . . . . . . . . . . . 10

2.3.2 Charge Transport in PEDOT:PSS . . . . . . . . . . . . . 12

2.3 .3 Doping PEDOT:PSS . . . . . . . . . . . . . . . . . . 13

3 Modelling \& Simulation $\quad 16$

3.1 Device Structure . . . . . . . . . . . . . . . . . . . . . . 16

3.2 Model Development . . . . . . . . . . . . . . . . . . . . . . . 17

3.3 Simulation . . . . . . . . . . . . . . . . . . . . . . . . . 19

3.4 Simulation Results . . . . . . . . . . . . . . . . . . 22

4 Fabrication 32

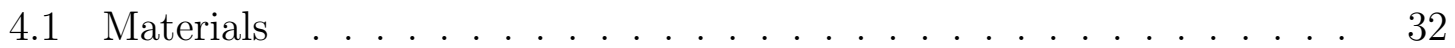

$4.2 \quad$ Fabrication Steps $\ldots \ldots \ldots \ldots \ldots$

4.3 Process Improvements . . . . . . . . . . . . . . . . . . . . . 37

5 Testing \& Results $\quad 38$

5.1 Testing Setup . . . . . . . . . . . . . . . . . . . . 38

5.2 Results . . . . . . . . . . . . . . . . . . . . . . . . . . 40

$5.2 .1 \quad$ Physical Properties . . . . . . . . . . . . . . . . 40

5.2 .2 Device Functionality . . . . . . . . . . . . . . . . . . . 43

5.3 Simulation and Measurement Comparison $\ldots \ldots \ldots \ldots 67$

6 Conclusion $\quad 74$

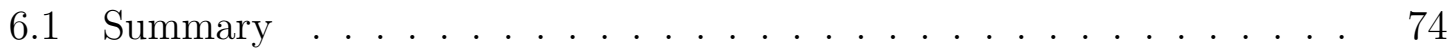

6.2 Future Work . . . . . . . . . . . . . . . . . . . 75

$\begin{array}{ll}\text { List of References } & 76\end{array}$ 
Appendix A SPICE Netlists for Simulations 


\section{List of Tables}

3.1 Variable values used in non-linear resistor simulations. . . . . . . . . 24

5.1 Summary of equipment used in testing. . . . . . . . . . . . . . . . . . 40

5.2 Physical device properties for Rail 1. . . . . . . . . . . . . . 41

5.3 Physical device properties for Rail 2. . . . . . . . . . . . . . . 42 


\section{List of Figures}

2.1 Connectivity in a rectangular CNN (modified from [2]). . . . . . . . . 4

2.2 Visualization of the four fundamental circuit elements and their relationships $[9] \ldots \ldots \ldots \ldots \ldots$

2.3 An example of a self-crossing hysteresis loop (modified from [11]). . . 8

2.4 Simplified device structure of HP memristor (from [12]). . . . . . . . 9

2.5 Ionic bonding structure of PEDOT chain (bottom) and PSS backbone (modified from [17]). . . . . . . . . . . . . . . . . . 11

2.6 Templated PEDOT:PSS and its formation into a film. (a) Chemical structure of ionically bonded PEDOT:PSS. (b) Segments of PEDOT interspersed along the PSS backbone. (c) Water saturated, entangled PSS chains forming larger, amorphous particles. $(d)$ Larger particles of tangled PEDOT:PSS chains creating a consistent film. (with per-

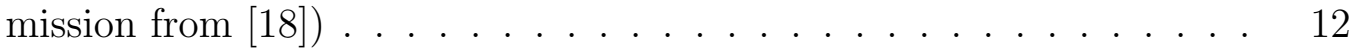

3.1 General structure and lumped elements model of non-linear resistor. . 16

3.2 Lumped elements model of dual-line transistor device (with permission from $[18]) \ldots \ldots \ldots \ldots \ldots$

3.3 Measured conductance of dual-line device compared to modelled curve (with permission from $[18]$ ). . . . . . . . . . . . . . . . . . . 19 
3.4 Voltage-controlled current source with custom gain expression (with permission from $[18]) \ldots \ldots \ldots \ldots \ldots$

3.5 One-dimensional conducting polymer cell with two dimensional ionic connections (with permission from $[18]$ ) . . . . . . . . . . 21

3.6 Two-dimensional conducting polymer cell with two dimensional ionic connections (with permission from $[18]$ ) . . . . . . . . . . 21

3.7 Several common configurations for ionic resistance blocks (with permission and modified from $[18]) \ldots \ldots \ldots \ldots$

3.8 Complete circuit schematic to simulate a strip of conductive polymer surrounded by electrolyte. . . . . . . . . . . . . . 2 23

3.9 Frequency sweep results for the one-line non-linear resistor model. . . 25

3.10 Simulation results of non-linear resistor device given a $10 \mathrm{~Hz}$ sinusoidal input of amplitude $5 \mathrm{~V} \ldots \ldots \ldots \ldots \ldots \ldots$

3.11 I-V curves measured across a non-linear resistor device at a frequency of $100 \mathrm{mHz}$ with varying amplitude $[25] . \ldots \ldots \ldots 27$

3.12 Simulation results for varying amplitudes of a sinusoidal input of frequency $100 \mathrm{mHz} . \ldots \ldots \ldots \ldots$

3.13 I-V curves measured across a non-linear resistor device at a frequency of $1 \mathrm{~Hz}$ with varying amplitude $[25] \ldots \ldots \ldots \ldots$

3.14 Simulation results for varying amplitudes of a sinusoidal input of frequency $1 \mathrm{~Hz} . \ldots \ldots \ldots \ldots \ldots \ldots \ldots \ldots$

3.15 I-V curves measured across a non-linear resistor device with varying frequency and a $3 \mathrm{~V}$ amplitude $[26] \ldots \ldots \ldots \ldots$

3.16 Simulation results for varying frequencies of a sinusoidal input of amplitude 5 V. . . . . . . . . . . . . . . . . . . . . 31 
4.1 Cross-section of PEDOT:PSS on PET on a glass substrate with header pins as contacts...................... 34

4.2 Cross-section of device in fabrication with silver ink applied to form reliable contact from pin to polymer. . . . . . . . . . . . . . .

4.3 Cross-section of device in fabrication where cured UV epoxy has been applied, insulating the contacts and forming the electrolyte well. . . .

4.4 Cross-section of device in fabrication where electrolyte has been deposited into the epoxy well. . . . . . . . . . . . . . . . . . . 36

4.5 Final cross-section of device showing all applied materials including epoxy seal on top of electrolyte well. . . . . . . . . . . . . . . . . 36

4.6 Photo of a mostly complete device. The blue strip in the middle is the PEDOT:PSS channel; the header pins and cured silver ink are visible at either end of the PEDOT:PSS strip. The epoxy that forms the device well can also be seen surrounding the device. No electrolyte has been deposited yet. . . . . . . . . . . . . . . . . . . . . 37

5.1 Test circuit configuration. . . . . . . . . . . . . . . . . 39

5.2 Side by side of device R1D1 with positive voltage applied to the left electrode with respect to the right electrode (top) and with no applied

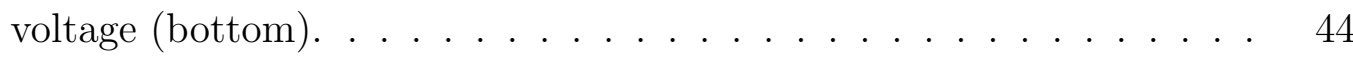

5.3 Side by side of device R1D1 with positive voltage applied to the right electrode with respect to the left electrode (top) and with no applied voltage (bottom). . . . . . . . . . . . . . .

5.4 Voltage over time for device R1D1 at $10 \mathrm{~Hz}$ and $5 \mathrm{~V}$ amplitude. . . . 46

5.5 I-V curve for device R1D1 at $10 \mathrm{~Hz}$ and $5 \mathrm{~V}$ amplitude. Arrows indicate signal change with respect to time. . . . . . . . . . . . . . . 46 
5.6 Device R1D2 with positive voltage applied to the left electrode with respect to the right electrode (top) and without (bottom). . . . . . . 48

5.7 Device R1D2 with positive voltage applied to the right electrode with respect to the left electrode (top) and without (bottom). . . . . . . . 49

5.8 Voltage over time for testing of device R1D2 at $10 \mathrm{~Hz}, 3 \mathrm{~V}$ amplitude. $\quad 50$

5.9 I-V curve for R1D2 at $10 \mathrm{~Hz}$. Arrows indicate signal change over time. 50

5.10 Device R1D4 with positive voltage applied to the left electrode with respect to the right electrode (top) and without (bottom). . . . . . 51

5.11 Device R1D4 with positive voltage applied to the right electrode with respect to the left electrode (top) and without (bottom). . . . . . . 52

5.12 Voltage over time for testing of device R1D4 at $10 \mathrm{~Hz}, 3 \mathrm{~V}$ amplitude. 53

$5.13 \mathrm{I}-\mathrm{V}$ curve for device R1D4 at $10 \mathrm{~Hz}$. . . . . . . . . . . . . . . . 53

5.14 Device R1D5 with positive voltage applied to the left electrode with respect to the right electrode (top) and without (bottom). . . . . . . 55

5.15 Device R1D5 with positive voltage applied to the right electrode with respect to the left electrode (top) and without (bottom). . . . . . . 55

5.16 Voltage over time for testing of device R1D5 at $10 \mathrm{~Hz}, 3 \mathrm{~V}$ amplitude. 56

$5.17 \mathrm{I}-\mathrm{V}$ curve for device R1D5 at $10 \mathrm{~Hz}$. . . . . . . . . . . . . 56

5.18 Device R2D2 with positive voltage applied to the left electrode with respect to the right electrode (top) and without (bottom). . . . . . . 57

5.19 Device R2D2 with positive voltage applied to the right electrode with respect to the left electrode (top) and without (bottom). . . . . . . 58

5.20 Voltage over time for testing of device R2D2 at $10 \mathrm{~Hz}, 3 \mathrm{~V}$ amplitude. 59

$5.21 \mathrm{I}-\mathrm{V}$ curve for device R2D2 at $10 \mathrm{~Hz} \ldots \ldots \ldots$. . . . . . . . . . . 59

$5.22 \mathrm{I}-\mathrm{V}$ curve for device R2D2 at $20 \mathrm{~Hz} \ldots \ldots \ldots$. . . . . . . . . 60 
5.23 Device R2D3 with positive voltage applied to the left electrode with respect to the right electrode(top) and without (bottom). . . . . . . 61

5.24 Device R2D3 with positive voltage applied to the right electrode with respect to the left electrode (top) and without (bottom). In the top half of the photo, a needle was used to distort the top of the electrolyte and reduce the shine so that the ion front could be seen. . . . . . . . 62

5.25 Voltage over time for testing of device R2D3 at $10 \mathrm{~Hz}, 2.5 \mathrm{~V}$ amplitude. 63

$5.26 \mathrm{I}-\mathrm{V}$ curve for device R2D3 at $10 \mathrm{~Hz}$. . . . . . . . . . . . . . 63

5.27 Device R2D4 with positive voltage applied to the left electrode with respect to the right electrode (top) and without (bottom). . . . . . . 64

5.28 Device R2D4 with positive voltage applied to the right electrode with respect to the left electrode (top) and without (bottom). . . . . . . 65

5.29 Voltage over time for testing of device R2D4 at $10 \mathrm{~Hz}, 2.5 \mathrm{~V}$ amplitude. 66

$5.30 \mathrm{I}-\mathrm{V}$ curve for device R2D4 at $10 \mathrm{~Hz} \ldots \ldots . . \ldots 66$

5.31 Simulation circuit schematic used for comparison to real device tests to account for sensing resistor. . . . . . . . . . . . . . . 68

$5.32 \mathrm{I}-\mathrm{V}$ characteristic curves for simulated devices with varying frequency inputs. ......................... 70

$5.33 \mathrm{I}-\mathrm{V}$ characteristic curves measured on device R2D2 with varying frequency inputs. . . . . . . . . . . . . . . . . . . 70

$5.34 \mathrm{I}-\mathrm{V}$ characteristic curves for simulated devices with varying voltage amplitude inputs to compare with R1D5. . . . . . . . . . . . . . . 72

$5.35 \mathrm{I}-\mathrm{V}$ characteristic curves measured on device R1D5 with varying voltage amplitude inputs. . . . . . . . . . . . . . . . . 72

5.36 I-V characteristic curves for simulated devices with varying voltage amplitude inputs to compare with R2D3. . . . . . . . . . . . 73 
5.37 I-V characteristic curves measured on device R2D3 with varying voltage amplitude inputs. . . . . . . . . . . . . . . . 73 


\section{Abbreviations and Symbols}

$I \quad$ Electric Current $[\mathrm{A}]$

$\varphi \quad$ Magnetic Flux Linkage [Wb, V-s]

$q \quad$ Electric Charge $[\mathrm{C}]$

$V \quad$ Electric Potential [V]

CNN Cellular Neural Network

DUT Device Under Test

nn-H Nearest-Neighbour Hopping

PC Propylene Carbonate

PEDOT Poly(3,4-ethylenedioxythiophene)

PEDOT:PSS Poly(3,4-ethylenedioxythiophene):Poly(styrenesulfonate)

PET Polyethylene Terephthalate

VCCS Voltage-Controlled Current Source

VRH Variable Range Hopping 


\section{Chapter 1}

\section{Introduction}

\section{$1.1 \quad$ Objectives}

The primary objective of the work in this thesis is to create a simple and efficient method to simulate ionically-coupled, non-linear devices with reasonable accuracy that could be scaled up for use in modelling larger, more complex neuropmorphic circuits, as well as to verify the accuracy of these simulations via the fabrication and testing of these devices.

\subsection{Outline}

A brief background relating to cellular neural networks, the discovery and creation of the memristor are discussed in Chapter 2. In addition, a look into memristor-like devices is presented, followed by a background of some of the more significant materials used in this thesis. Chapter 3 investigates the device structure of memristor-like devices and how it can be developed into a practical mathematical model which can implemented in a reasonably accurate, if approximated, manner. A method for fabricating these devices in presented in Chapter 4. The results of these simulated 
models are discussed in Chapter 5 and their usefulness determined through comparison to results from previous work within the research group. Next, a practical method for fabricating these devices is presented using simple methods to create large memristor-like devices. Finally, the structure and operation of several promising devices is evaluated, and the behaviour of these devices is compared to the previously developed simulation for validation. 


\section{Chapter 2}

\section{Background}

In the following sections, an introduction to cellular neural networks is presented, followed by a discussion of their applications. A particular emphasis is placed on their role as neuromorphic networks, as the devices investigated for this thesis are ultimately intended to function as elements in such a network. A discussion of nonlinear elements follows as a means to demonstrate practical examples of useful CNN cells. Subsequently, conductive polymers and their usefulness in creating non-linear elements is also presented.

\subsection{Cellular Neural Networks}

A cellular neural network, or CNN, is a multidimensional array of mainly identical dynamical systems called "cells". Each cell is restricted to interactions only within its own local space within a finite radius, typically referred to as "nearest neighbour" interactions. Additionally, all state variables in the CNN are continuous signals [1]. Cells can only communicate with distant cells in an indirect manner by propagating their input through their nearest neighbours and allowing each cell to process the data independently, based on how the CNN is programmed. Figure 2.1(a) shows the 
general form of a nearest-neighbour CNN. In Figure 2.1(b), a symbolic notation for a computing cell is presented where the state of the cell, $X_{i j}$, is influenced by $I_{i j}$ which is a summation via Kirchoff's Current Law of the nearest-neighbour contributions. Stationary and time-varying input signals are denoted by $U_{i j}$ and $u_{i j}(t)$ respectively, and $y_{i j}$ represents the output of the cell at position $(i, j)$.

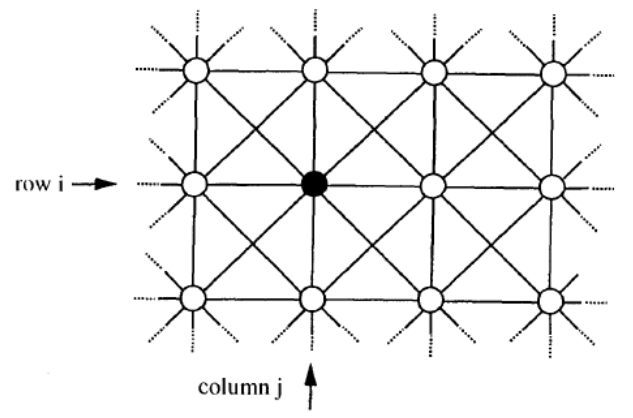

(a)

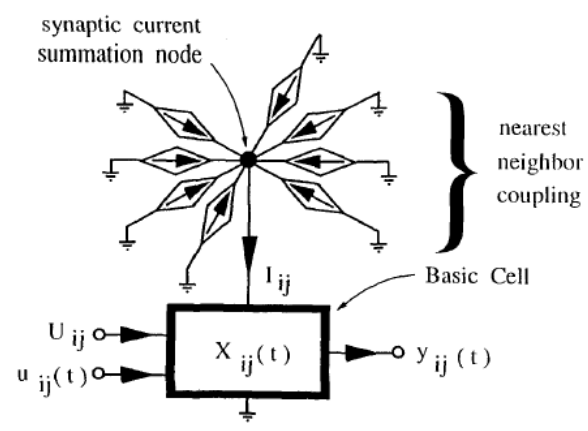

(b)

Figure 2.1: Connectivity in a rectangular CNN (modified from [2]).

The set of parameters, arranged in matrices, that define a CNN of any size are shown in (2.1), where $\dot{\mathbf{x}}$ is array for the time derivative of $\mathbf{x}$.

$$
\dot{\mathrm{x}}=-\mathrm{x}+\mathrm{Ay}+\mathrm{Bu}+\mathrm{U}
$$

A template is what is used to program the interactions between a cell and its adjacent cells and is known as the "cloning template" [2]. For example, the cloning template that would describe a basic cell in a simple grid arrangement with connections to its eight nearest neighbour cells is given in (2.2), where matrices $\mathbf{A}$ and $\mathbf{B}$ describe the feedback and feedforward templates, respectively, for any one cell in the network. 


$$
\mathbf{A}=\left[\begin{array}{ccc}
a_{i-1, j-1} & a_{i, j-1} & a_{i+1, j-1} \\
a_{i-1, j} & a_{i, j} & a_{i+1, j} \\
a_{i-1, j-1} & a_{i, j-1} & a_{i+1, j+1}
\end{array}\right], \mathbf{B}=\left[\begin{array}{ccc}
b_{i-1, j-1} & b_{i, j-1} & b_{i+1, j-1} \\
b_{i-1, j} & b_{i, j} & b_{i+1, j} \\
b_{i-1, j-1} & b_{i, j-1} & b_{i+1, j+1}
\end{array}\right]
$$

Upon receiving an external input, each cell will process the stimuli it detects as well as the output of its nearest neighbours, and will propagate its resultant signal to each of its own nearest neighbours, which will themselves repeat the process until the edge of the network is reached. In this way, a CNN will always settle to a specific steady state after receiving an input. A global effect is reached through several iterations of the local filter for a cell, through what is known as the "propagation property" [3].

\subsubsection{Applications of Cellular Neural Networks}

Due to their parallel structure, CNNs can process certain types of data very quickly. This makes them particularly competent at several specific applications. One of the most evident, and commonly explored, is image processing [4]. Another common application of the cellular neural network is a class of electronic network referred to as a neuromorphic network. A neuromorphic network is designed to emulate the architecture and operation of biological systems [5]. These systems include such human equivalences as the retina of the eye [6] or the neurons in the brain [7].

\subsection{Non-Linear Resistive Elements}

One type of device that is particularly well-suited for use as a cell unit in a CNN is the memristor, since it fulfils the necessary requirements of CNN elements, such as being continuous, dynamic devices that can be arranged into a multidimensional 
array and only require connections to their nearest neighbour cells. Non-linearity is also a necessary feature for CNN cells that memristors inherently possess.

\subsubsection{Memristors}

A memristor is a passive, two-terminal circuit element. It was first posited by Dr. Leon Chua in an article in 1971 [8]. The basis of his argument for the existence of the memristor (so named as a portmanteau of "memory" and "resistor") was that there were six mathematical relations relating pairs of the four fundamental electronic circuit variables: voltage $v$, current $i$, charge $q$, and magnetic flux linkage $\varphi$. Two of these pairs are time dependent, one defining the concept of flux and the other the concept of induction. Of the four remaining relations, three of them define the three

common passive devices (resistor, capacitor, and inductor), leaving one relationship without a corresponding device. These relationships are summarized in a visual form in Figure 2.2.

The mathematical relations not yet assigned to a passive device are what define the memristor, summarized in (2.3).

$$
\mathrm{d} \varphi=M \mathrm{~d} q
$$

Equation (2.3) can also be used to relate the voltage and current through a "charge-controlled" memristor, as shown in (2.4). A similar relation is valid for a "flux-controlled memristor".

$$
v(t)=M(q(t)) i(t), M(q)=\frac{\mathrm{d} \varphi(q)}{\mathrm{d} q}
$$

$M$ is a function of charge and represents the "memristance" of the device. From 


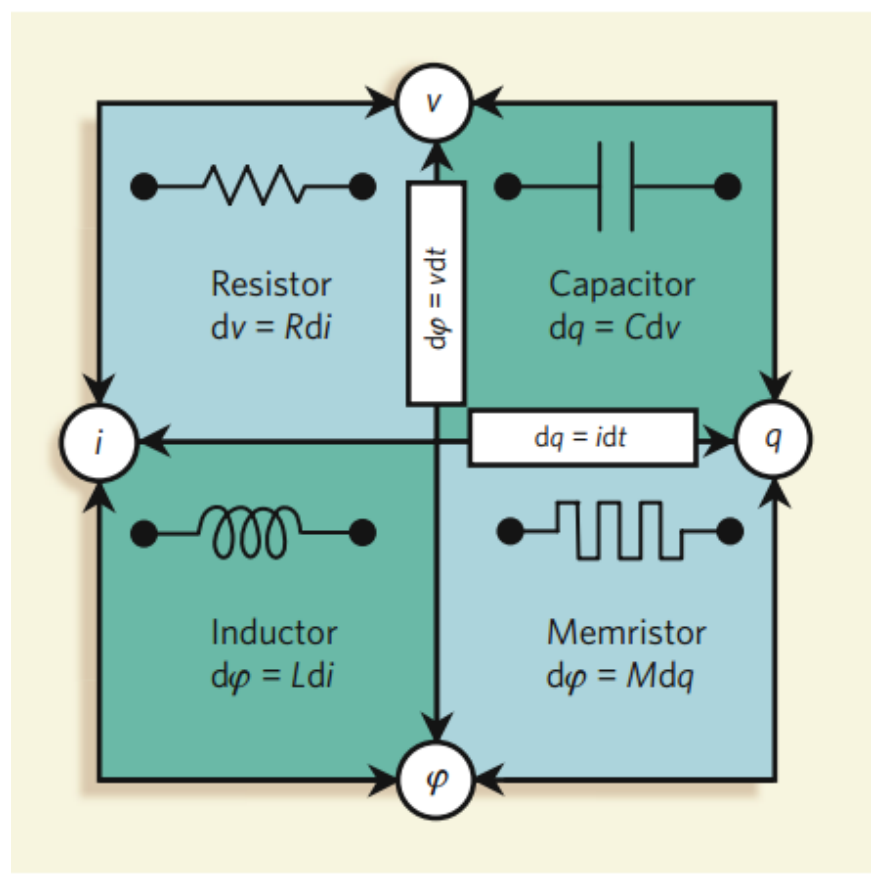

Figure 2.2: Visualization of the four fundamental circuit elements and their relationships [9].

this, it can be seen that a memristor with constant current will simply be a resistor. The $i-v$ characteristic for a sinusoidal input to a typical memristor generally takes the form of a frequency dependent Lissajous figure [10]. This shape is often described as a "pinched hysteresis" curve. There are two types of hysteresis loops, referred to as "self-crossing" and "not self-crossing" [11]. An example of the self-crossing pinched hysteresis curve characteristic of a memristor is shown in Figure 2.3. Along with the concept of the memristor, Chua also proposed several practical circuit realizations of the memristor using the existing passive elements combined with active components.

In 1976, Chua published a follow-up paper which expanded the definition of memristors to include what he named "memrsitive systems" [10]. This definition included any device or system whose characteristics were similar to that of the memristor, though they cannot be accurately modelled strictly with classic memristor theory. 


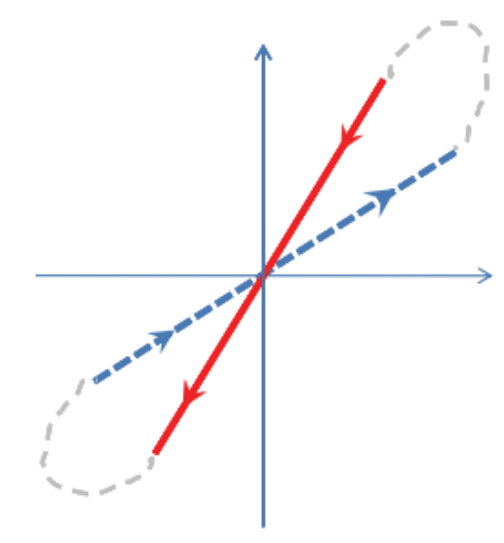

Figure 2.3: An example of a self-crossing hysteresis loop (modified from [11]).

These systems were defined by state equations, shown in (2.5), where $u$ and $y$ represent the input and output of the system, and $x$ represents the state of the system.

$$
\begin{gathered}
\dot{x}=f(x, u, t) \\
y=g(x, u, t) u
\end{gathered}
$$

These state equations can be used to describe far more systems than those represented in the original article.

\subsubsection{The First Memristors}

The first discrete, passive, 2-terminal devices that were claimed to be memristors were developed by HP Labs in 2008. The device is based on dopant movement in a $5 \mathrm{~nm}$ titanium dioxide active layer sandwiched between two metal contacts [12]. The device is a semiconductor of thickness $D$ between two metal contacts, in which there are two variable resistors in series. One resistor is the area of the film that has a high concentration of dopants, and is labelled $R_{O N}$. The other region, having low dopant concentration, is labelled $R_{O F F}$. This structure is shown in Figure 2.4, where the 
doped region is $R_{O N}$ and the undoped region is $R_{O F F}$.

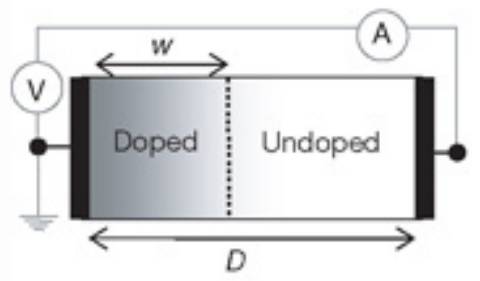

Figure 2.4: Simplified device structure of HP memristor (from [12]).

Applying a voltage across the device moves the boundary between the two regions by causing the dopants to drift through the material. Using standard ion drift equations and device dimension and fitting them into the memristive system state equations, an expression for the memristance of this structure is obtained, as shown in $(2.6)$.

$$
M(q)=R_{O F F}\left(1-\frac{\mu_{V} R_{O N}}{D^{2}} q(t)\right)
$$

where $\mu_{V}$ is the average ion mobility. The state variable for the equation represented by the dopant distribution term is, naturally, bounded between 0 and the device thickness, $D$. This restricts the device operation and defines the "memristor regime", outside of which the device cannot be considered a memristor. As long as the device is operated within these bounds, a symmetrical, double loop hysteresis $i-v$ curve is seen as the result of a sinusoidal input. As it should, the hysteresis loop becomes narrower as frequency is increased, ultimately reaching a linear relationship.

\subsubsection{Memristor-like Devices}

There has been some level of controversy regarding HP's 2008 announcement of their discovery of the memristor. Primarily, the arguments centre on the fact that HP's 
memristive devices do not interact in any meaningful way with magnetism, and that Chua's original paper implied that this magnetic interaction was significant as part of the device's operation [13]. Some claim that these resistive memory elements are simply a broader category of device that the memristor would then fit somewhere within. These arguments have been refuted by Chua, who claims that all two-terminal resistance switching memory devices are memristors [14]. It is generally accepted, however, that the devices discussed in 2008 at least fall within the more general classification of memristive systems.

\subsection{Conductive Polymers}

This section discusses some of the materials used in the construction of the devices created for this thesis. It will focus on the conductive polymer PEDOT:PSS and discuss the mechanism of conduction therein, before discussing dynamic doping of the material.

\subsubsection{PEDOT:PSS}

Bayer AG research laboratories developed a water-soluble polyelectrolyte called Poly(3,4-ethylenedioxythiophene):Poly(styrenesulfonic acid) (abbreviated PEDOT:PSS) in the 1980s. It has been found to have a wide array of applications, from antistatic layers for photographic films, to electroluminescent displays, to organic transistors [15]. Part of the reason why PEDOT:PSS has seen so many applications is that it can be incorporated into a design process in a wide variety of ways including: screen-printing, ink jet printing, spin coating, and vacuum deposition [16].

The PEDOT chain can lose an electron from the EDOT monomer to take on a positive charge, becoming what is known as a polycation. The PSS chain forms 
a polyanion by losing a hydrogen atom from its $\mathrm{SO}_{3} \mathrm{H}$ group. The PSS polyanion can oxidize an EDOT monomer, creating an ionic bond between them and thereby p-doping the PEDOT chain. This bonding structure is shown in Figure 2.5.

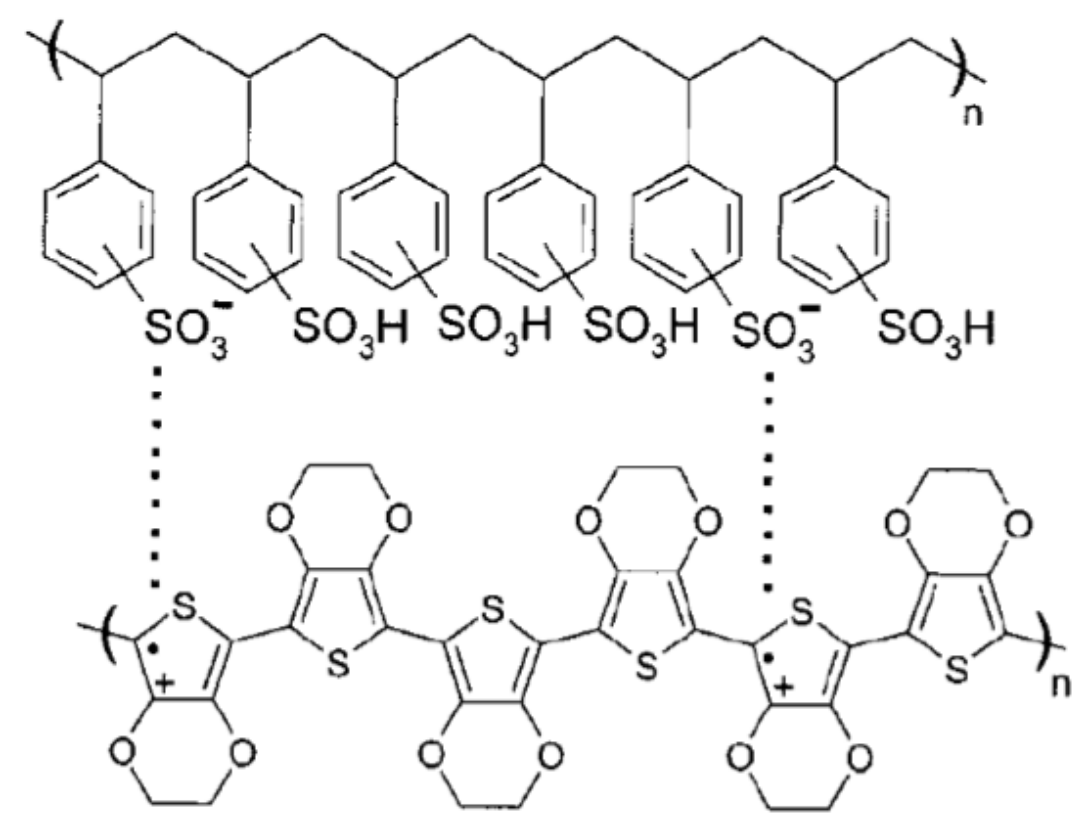

Figure 2.5: Ionic bonding structure of PEDOT chain (bottom) and PSS backbone (modified from [17]).

In addition, it is possible to use a PSS backbone as a template to grow PEDOT on, as EDOT monomers oxidize and bond onto it, attaching the two polymers and resulting in a p-doped, conductive final product. The PEDOT does not form a continuous polymer bond along the entire length of the PSS backbone, however. Instead it forms segmented chains of PEDOT intermittently attached along the length of the PSS backbone, as can be seen in Figure 2.6(b) [15]. Since these sections of PEDOT:PSS are doped, they form conductive sections capable of accepting electrons from other conductive sections. Lengths of PSS with sporadically bonded PEDOT can then be immersed in water, allowing them to exist in a liquid film, suitable for various deposition techniques, as seen in Figure 2.6(d). 


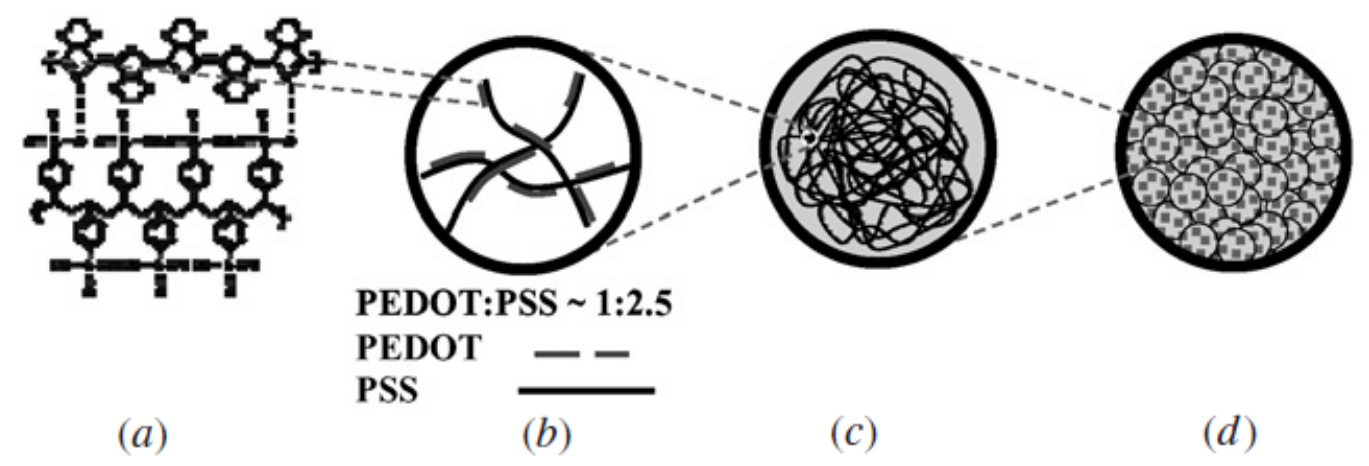

Figure 2.6: Templated PEDOT:PSS and its formation into a film.

(a) Chemical structure of ionically bonded PEDOT:PSS.

(b) Segments of PEDOT interspersed along the PSS backbone.

(c) Water saturated, entangled PSS chains forming larger, amorphous particles.

(d) Larger particles of tangled PEDOT:PSS chains creating a consistent film. (with permission from [18])

\subsubsection{Charge Transport in PEDOT:PSS}

A PEDOT:PSS film can be visualized as an irregular composition of conductive and less conductive regions. The conductive regions are where there is a large concentration of PEDOT-rich particles, whereas the non-conductive regions are primarily composed of only the PSS backbone material [19]. Charge transport in this material is heavily dependent on the transport of charge within and between these regions. The conductivity, then, is strongly based on the ratio of PEDOT to PSS (i.e. the density of PEDOT-rich areas) [20]. In a similar manner, the conductivity of the film can also be reduced by adding other non-conductive material to the film [21]. The conductivity can also be affected by other factors, such as a different backbone polymer, the level of doping added to the film, as well as the type of ion used to dope the film.

The morphology of conductive polymers makes it difficult to accurately model exactly how charge travels through the material. The charge transport models of 
traditional inorganic crystalline semiconductors or metals do not apply to the amorphous and random nature of conjugated polymers [21]. Electrons in those materials do not undergo a significant amount of scattering as they travel through the material, and as a result the electrons in those materials travel in a delocalised (i.e. free to move throughout the material) manner. This disorderly nature of conductive polymers means that electron scattering becomes a significant factor when considering the nature of electron movement through the material. Generally, electrons in a conductive polymer are no longer delocalised and become bound within a certain region of the material.

The result is that the conductivity model of conductive polymers is dominated by charge hopping effects [21]. This effect is known as Variable Range Hopping (VRH). It has been shown that the primary charge transport mechanisms in spin-coated PEDOT:PSS is three dimensional VRH and nearest-neighbour hopping (nn-H), depending on whether the charges are travelling within PEDOT-rich regions or between them [22].

\subsubsection{Doping PEDOT:PSS}

One trait which conductive polymers share with traditional semiconductors is the ability to be either p-doped (by losing an electron) or n-doped (by gaining an electron), greatly affecting their overall conductivity [21]. In PEDOT:PSS, the presence of dopants adding or removing electrons from the ionic bond between the PSS backbone and PEDOT sections results in the creation of a quasi-particle known as a bipolaron [23]. Bipolarons are the result of a pair of polarons, themselves a pair of solitons, which are created when an electron is removed from a polymer chain and a hybrid bond is created to accommodate this change, manifesting physically as a "kink" in the polymer chain. These quasi-particles are mobile along the polymer chains as well 
as between chains, so inter-chain conductivity remains the primary means of charge movement in a doped polymer.

Dopants can add or remove this charge via reduction or oxidation reactions, respectively, not by the addition of an impurity as in the case of crystalline semiconductors. These types of reactions are referred to as "redox" reactions.

A conductive polymer can be doped through a variety of means, including chemically, electrochemically, through photoinducing, and charge injection. The doping method used for this thesis was electrochemical doping. Electrochemical doping, in its simplest form, can be implemented in a very similar manner to the process of electro-plating. Two electrodes are placed into a solution of electrolyte, forming a circuit through the electrolyte. A potential is then applied across the electrodes and the cations and anions from the electrolyte separate and begin moving towards the electrodes. The electrode with the higher voltage, the cathode, attracts the anions whereas the lower potential electrode, the anode, draws the cations towards it. Depending on the type of doping desired, the polymer is placed on the corresponding electrode to have the appropriate type of ions drawn into it and cause redox reactions, doping the material. This process of reversibly inserting ions into a solid without affecting the structure is known as "intercalation" [24].

In the case of PEDOT:PSS, the PSS monomers drop their hydrogen atom, becoming $\mathrm{PSS}^{-}$, and oxidizing the PEDOT chain by forcing it to lose an electron and become p-doped, as seen in (2.7).The doped PEDOT ${ }^{+}$and $\mathrm{PSS}^{-}$counter ion then ionically bond to form stable PEDOT:PSS.

$$
\mathrm{PEDOT}^{0}+\mathrm{PSS}^{-} \rightarrow\left(\mathrm{PEDOT}^{+}: \mathrm{PSS}^{-}\right)^{0}+\mathrm{e}^{-}
$$

This doping of $\mathrm{PEDOT}^{+}$can be reversed by introduction of a cation such as $\mathrm{Li}^{+}$. 
The cation breaks the ionic bond between $\mathrm{PEDOT}^{+}$and $\mathrm{PSS}^{-}$and the $\mathrm{PEDOT}^{+}$ gains an electron and becomes dedoped, while the $\mathrm{Li}^{+}$cation neutralizes the $\mathrm{PSS}^{-}$ polyanion. Thus it can be said that the $\mathrm{LI}^{+}$cation reduces the $\mathrm{PEDOT}^{+}$, as is shown in $(2.8)[23]$.

$$
\left(\mathrm{PEDOT}^{+}: \mathrm{PSS}^{-}\right)^{0}+\mathrm{Li}^{+}+\mathrm{e}^{-} \rightarrow \mathrm{PEDOT}^{0}+\left(\mathrm{Li}^{+}: \mathrm{PSS}^{-}\right)^{0}
$$

These redox reactions can be applied dynamically to a section of conductive polymer by applying a potential across a section of PEDOT:PSS that is adjacent to mobile ions, intercalating $\mathrm{Li}^{+}$cations into the PEDOT:PSS and dedoping part of it. As the potential changes, so does the doping level, thus introducing a non-linear history aspect to what is otherwise a simple strip of conductive material. This principle forms the basis for the devices used in this thesis. 


\section{Chapter 3}

\section{Modelling \& Simulation}

In this chapter, a brief summary of the methods being implemented for modelling non-linear resistive devices is provided. An overview of other simulation work in this research group follows before a discussion of the methods implemented for this thesis.

\subsection{Device Structure}

The intended structure of the memristor-like device is very simple and straightforward. This structure is demonstrated in Figure 3.1; a single strip of conductive polymer (grey) is connected lengthways between two electrodes (black).

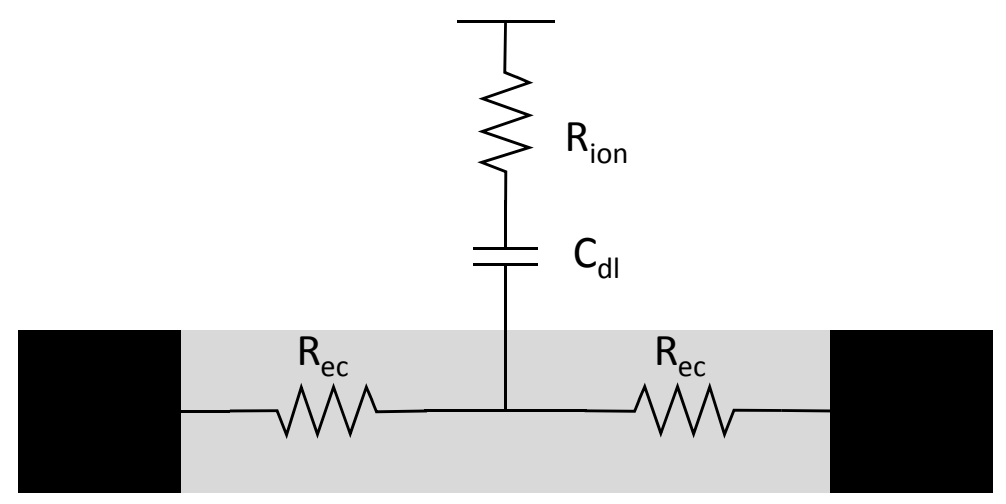

Figure 3.1: General structure and lumped elements model of non-linear resistor. 
The conductive polymer is immersed in ion-rich electrolyte and a path to intercalate ions is available. The electrodes are isolated from the electrolyte such that the only path from electrode to electrolyte is through the conductive polymer.

\subsection{Model Development}

The model and simulation method designed to simulate the devices in this thesis were based on the model and simulation method developed for a PEDOT:PSS-based dual-line transistor device, which used the same materials and electrochemical doping technique that was previously studied within the research group [18]. This transistor device works as an effective basis for a model of the non-linear resistors in this thesis because the geometries are highly similar. The development of a model for the dual-line device inevitably develops a model for a single line of conductive polymer surrounded by electrolyte.

In order to get an accurate model for the device, a lumped components equivalent circuit was developed, shown in Figure 3.2. It can be seen that, since the non-linear resistor device is simply a single strip of conductive polymer surrounded by electrolyte, the lumped element model for the dual-line device can easily be applied to the single line. This single line lumped element model is shown in Figure 3.1.

In this model, $R_{e c}$ is the distributed resistance of the conductive polymer element, $R_{i o n}$ represents the resistance associated with the movement of ions in the electrolyte, and $C_{d l}$ is the distributed "double layer" capacitance that is the result of the interaction of the conductive polymer and electrolyte boundary, particularly the tendency for ions of opposing charge (with respect to the electrode) to be near the electrode due to the applied voltage.

The next step in developing the model was based on curve-fitting and parameter 


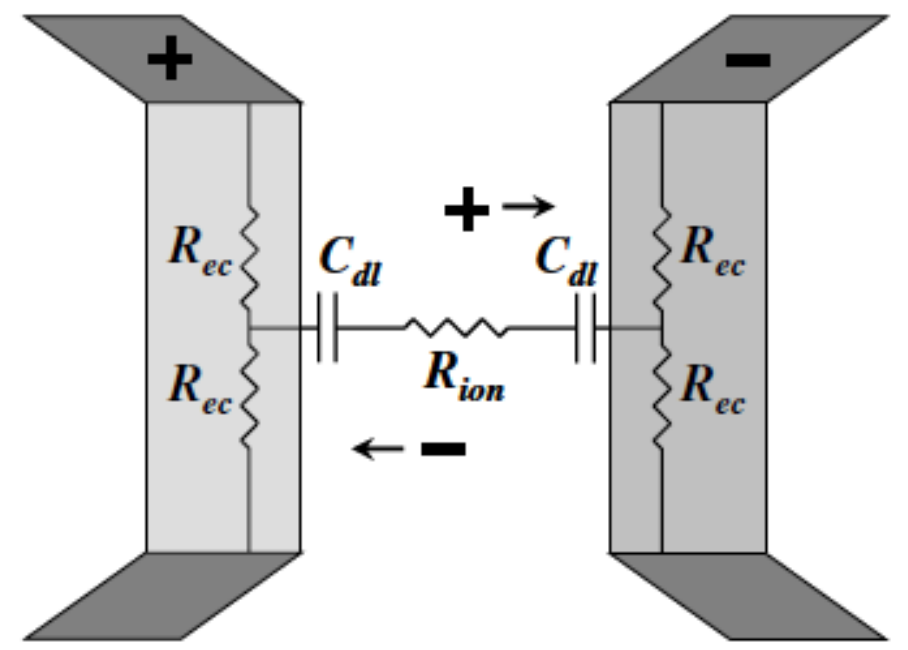

Figure 3.2: Lumped elements model of dual-line transistor device (with permission from [18]).

extraction of the dual-line transistor's conductivity response, which can be seen in Figure 3.3. The linear portion of the transfer characteristic can be given by (3.1).

A sigmoid curve was chosen to emulate the measured data, as shown in (3.2), where $G$ is the conductance of the material segment, $G_{0}$ is the maximum conductance, $G_{\min }$ is the minimum conductance, $C_{d l}$ is the double layer capacitance of the material segment, $Q_{d l}$ is the charge intercalation parameter, $V_{d l}$ is the voltage across the double layer capacitance (i.e. the potential difference between the conductive polymer and the electrolyte solution), and $V_{\text {off }}$ is the offset voltage at $\frac{G_{0}}{2}$.

$$
\begin{gathered}
Q_{a c c}=V_{a p p} \times C_{d l} \\
G=\frac{G_{0}}{1+\mathrm{e}^{\frac{C_{d l}\left(V_{d l}-V_{o f f}\right)}{Q_{d l}}}+G_{m i n}}
\end{gathered}
$$

Generally, $G_{\min }$ is small compared to $G_{0}$, and thus can usually be ignored. Neglecting $G_{m i n},(3.3)$ is the result. This equation characterizes the variable conductivity 


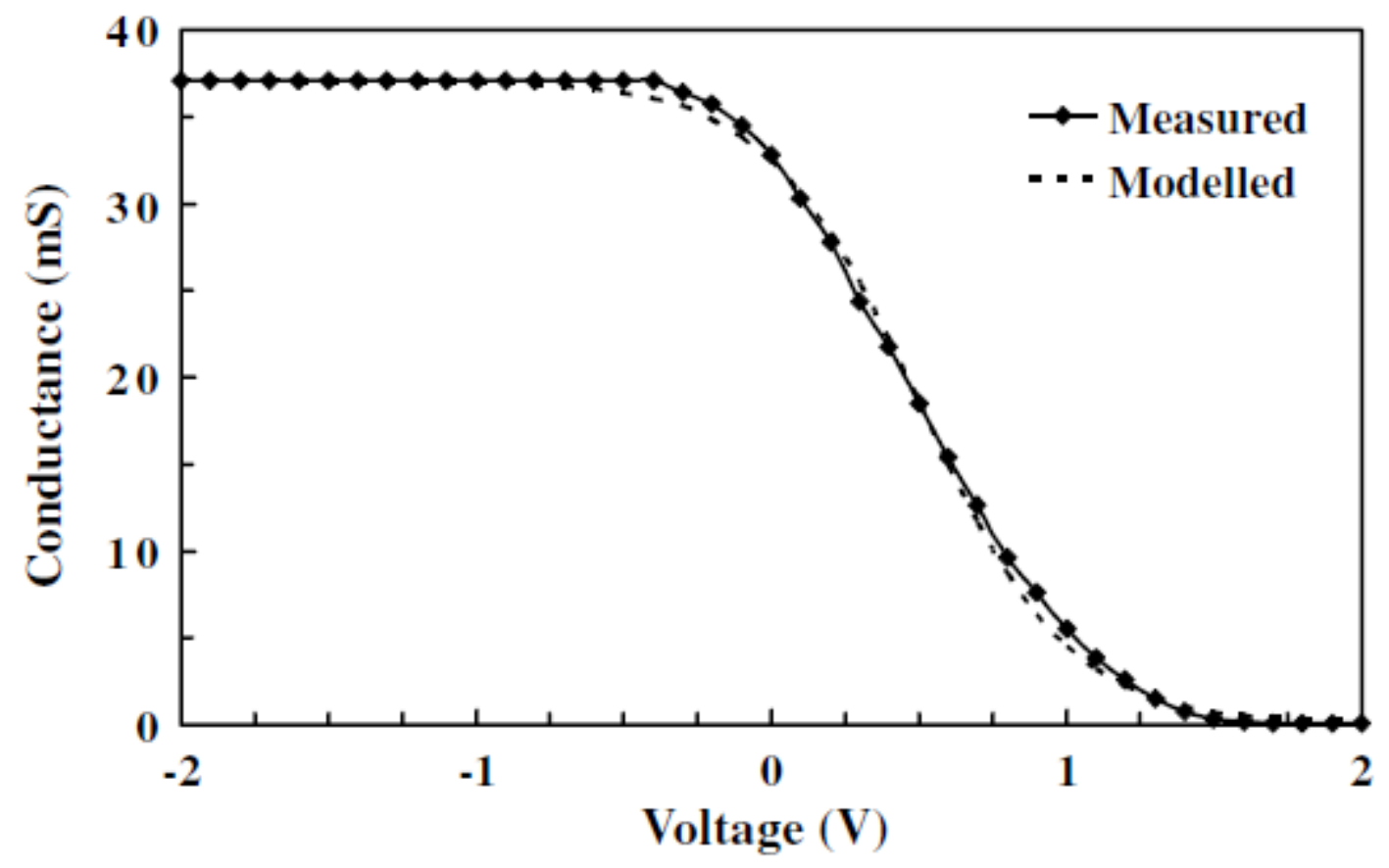

Figure 3.3: Measured conductance of dual-line device compared to modelled curve (with permission from [18]).

(or inversely, resistance) of a square of material and forms the basic building block used to simulate the non-linear resistors developed in this thesis.

$$
G=\frac{G_{0}}{1+\mathrm{e}^{\frac{C_{d l}}{Q_{d l}}\left(V_{d l}-V_{o f f}\right)}}
$$

\subsection{Simulation}

In order to generalize the geometry this model can be applied to, it is useful to separate the model into an electronic layer, which is made up of any active or passive electronic components, and an ionic layer which is made up of the electrolyte and any other ionic components. These layers interact with each other via the double layer capacitance at the boundary of the polymer and the electrolyte. In addition, potentially any geometry can be simulated by using the basic electronic layer model 
as a square, and connecting several squares to each other and to ionic layers.

The key component used to simulate the electronic layer blocks is a voltagecontrolled current source (VCCS) with a custom gain or transconductance expression, as shown in Figure 3.4. This element can be used to convert the voltage across the double layer capacitance into the conductivity for a short section of conductive polymer material. Combining this VCCS element with the lumped-element model previously established, a complete model for simulation is formed. This simulation cell for a one-dimensional conductive polymer element is shown in figure 3.5.

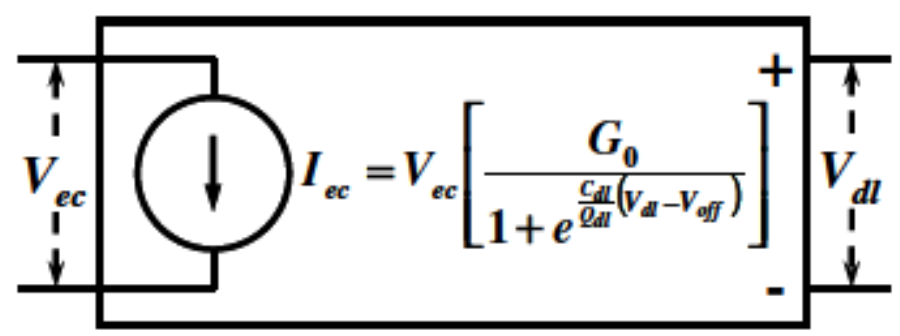

Figure 3.4: Voltage-controlled current source with custom gain expression (with permission from [18])

This cell can be used to represent a short section of conductive polymer that is surrounded by electrolyte solution. By connecting several 1D elements in series, a long strip of conductive polymer surrounded by electrolyte can be simulated. This cell can be expanded to a two-dimensional version that can be used to emulate virtually any geometry that can be constructed out of these standardized squares of material, and is shown in Figure 3.6.

The only remaining aspect of the device to simulate is the electrolyte surrounding the device. Given that the interaction between the device and the electrolyte is already defined by the VCCSs, all that needs to be done is to account for the resistance due to ion mobility. Thus, the electrolyte can be incorporated into the circuit as simple 


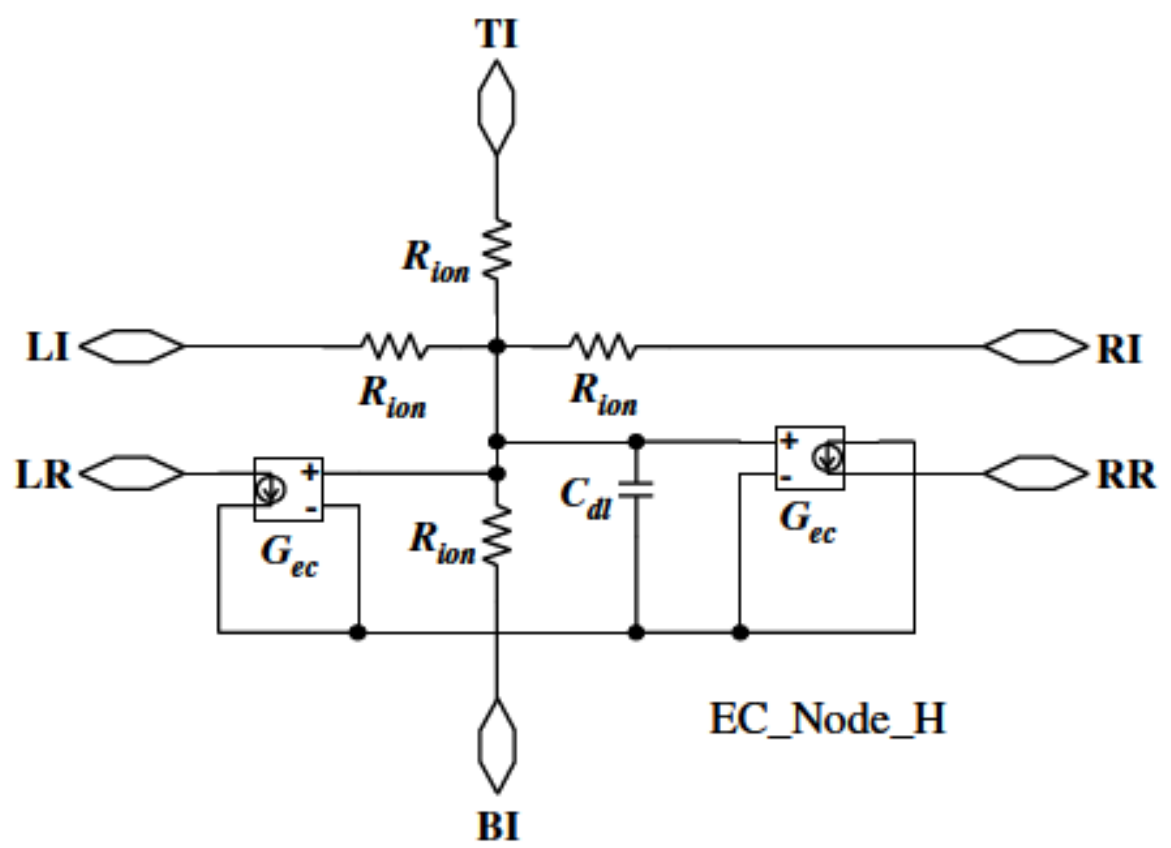

Figure 3.5: One-dimensional conducting polymer cell with two dimensional ionic connections (with permission from [18])

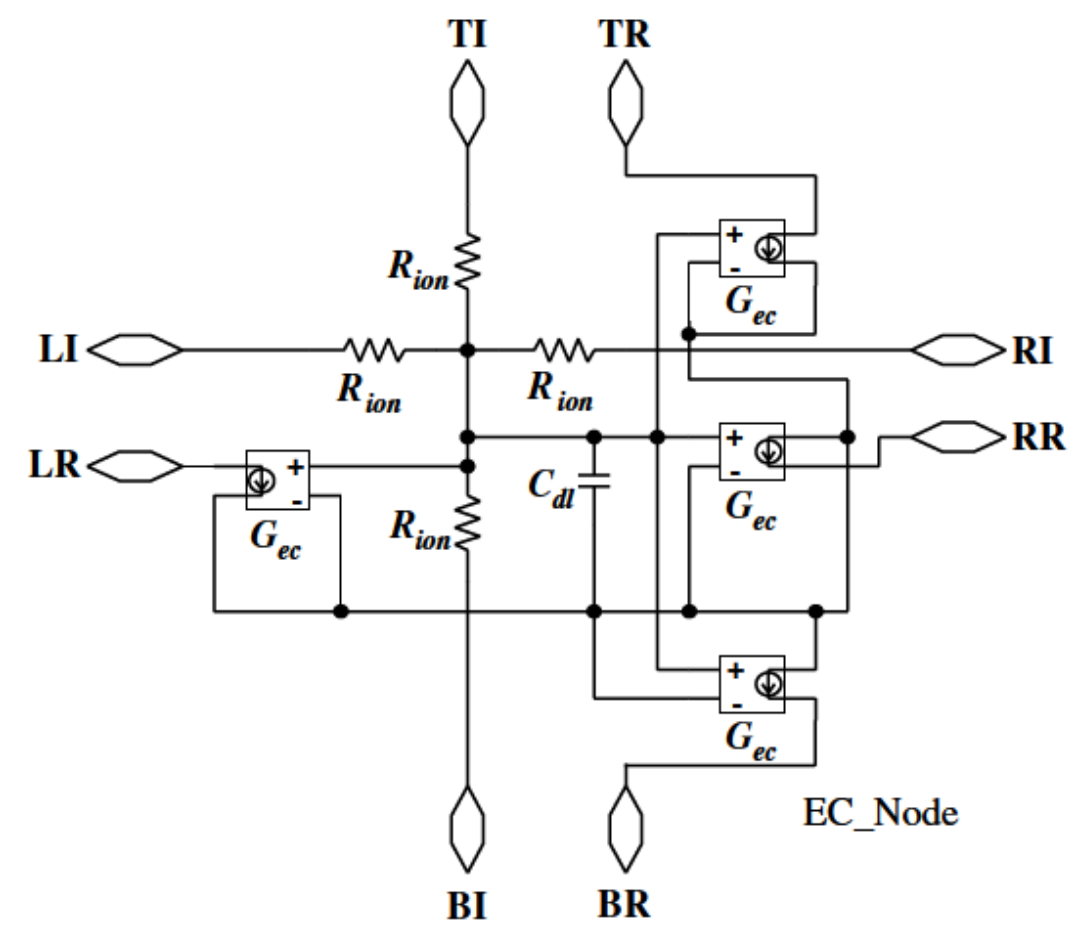

Figure 3.6: Two-dimensional conducting polymer cell with two dimensional ionic connections (with permission from [18]) 
resistors that connect to the ionic layer connections of the previously defined cells. To simplify simulation, several standard bocks representing common $R_{\text {ion }}$ configurations are added, with examples of what is contained in these blocks shown in Figure 3.7.

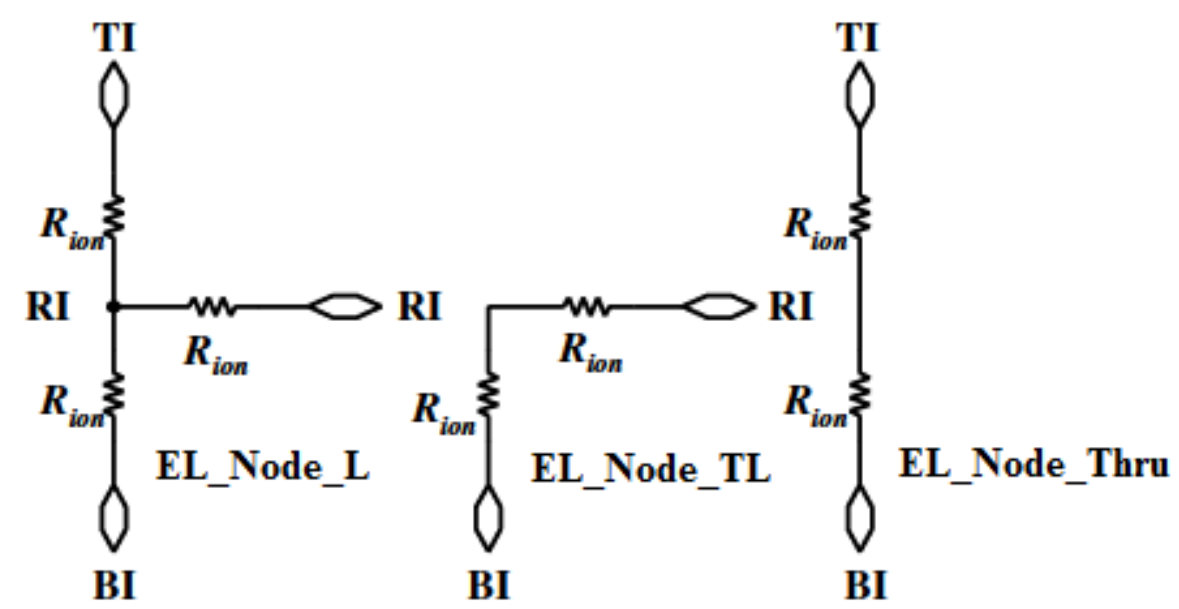

Figure 3.7: Several common configurations for ionic resistance blocks (with permission and modified from [18])

These elements can all be assembled into one circuit whose layout can accurately model the single-line variable resistor device used for this thesis. This circuit is shown in Figure 3.8. The sub-blocks for each component in the simulation were implemented by netlists which can be found in Appendix A. The variable values shown in Table 3.1 were used for all simulations in this chapter. These values were determined via parameter extraction to accurately represent the active material.

\subsection{Simulation Results}

In order to obtain the optimal response, a sinusoidal input with an amplitude of $5 \mathrm{~V}$ was applied while the frequency of the input signal was swept across a range of $0.1 \mathrm{~Hz}$ 


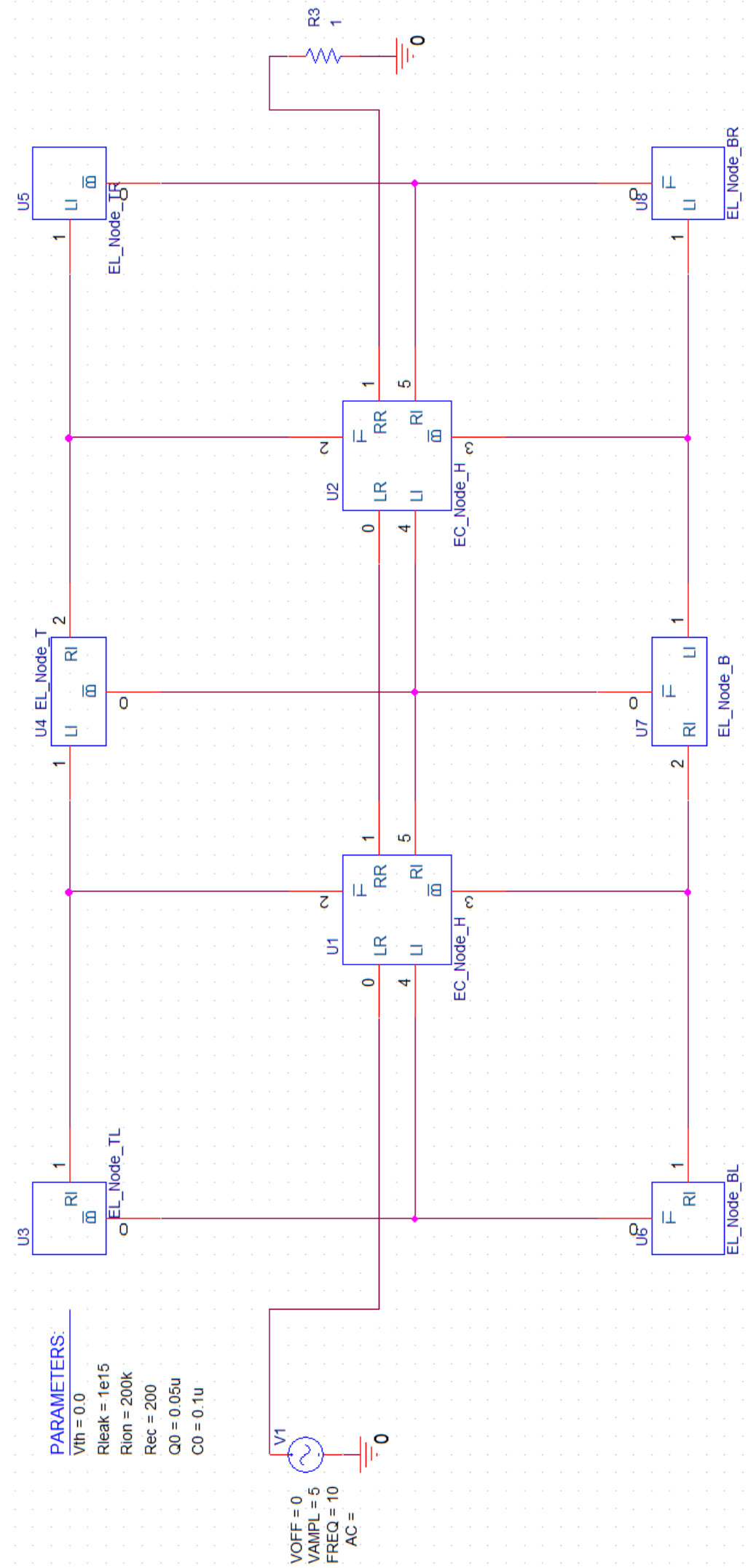

Figure 3.8: Complete circuit schematic to simulate a strip of conductive polymer surrounded by electrolyte. 
Table 3.1: Variable values used in non-linear resistor simulations.

\begin{tabular}{ll}
\hline Variable & Value \\
\hline$R_{\text {ec }}$ & $200 \Omega$ \\
$R_{\text {ion }}$ & $200 \mathrm{k} \Omega$ \\
$R_{\text {leak }}$ & $1 \times 10^{15} \Omega$ \\
$Q_{0}$ & $10 \mu \mathrm{C}$ \\
$C_{0}$ & $20 \mu \mathrm{F}$ \\
$V_{t h}$ & $0 \mathrm{~V}$ \\
\hline
\end{tabular}

to $100 \mathrm{~Hz}$ to determine the frequency which gives the best response. In this case, the best response is determined by the widest hysteresis loop, since that indicates more non-linearity and more significant memory effects. The plot of the $I-V$ curves for all tested frequencies is shown in Figure 3.9.

From this figure, it can be seen that the most non-linear response occurs with an input frequency of $10 \mathrm{~Hz}$. Due to this, all future simulations will be using an input frequency of $10 \mathrm{~Hz}$ in this chapter. Figure 3.10 shows the basic response of the device given a $10 \mathrm{~Hz}$ sinusoidal input with an amplitude of $5 \mathrm{~V}$. In addition, arrows have been added to the figure to show the movement of the signal over time. It is clear that this hysteresis effect is of the "not self-crossing" type. Also of note is the small curve on the inside of the loop in the first quadrant. This loop is due to a "start-up" effect as the ion intercalation is not as fast on the first cycle.

The most relevant point of comparison for the results of these simulations would be a master's thesis previously completed in this research group in which similar nonlinear devices were constructed and tested [25]. Their structure was similar enough that the behaviour of the devices should be very similar, but there will be some differences as the physical dimensions of the devices are different. Thus, the simulation 


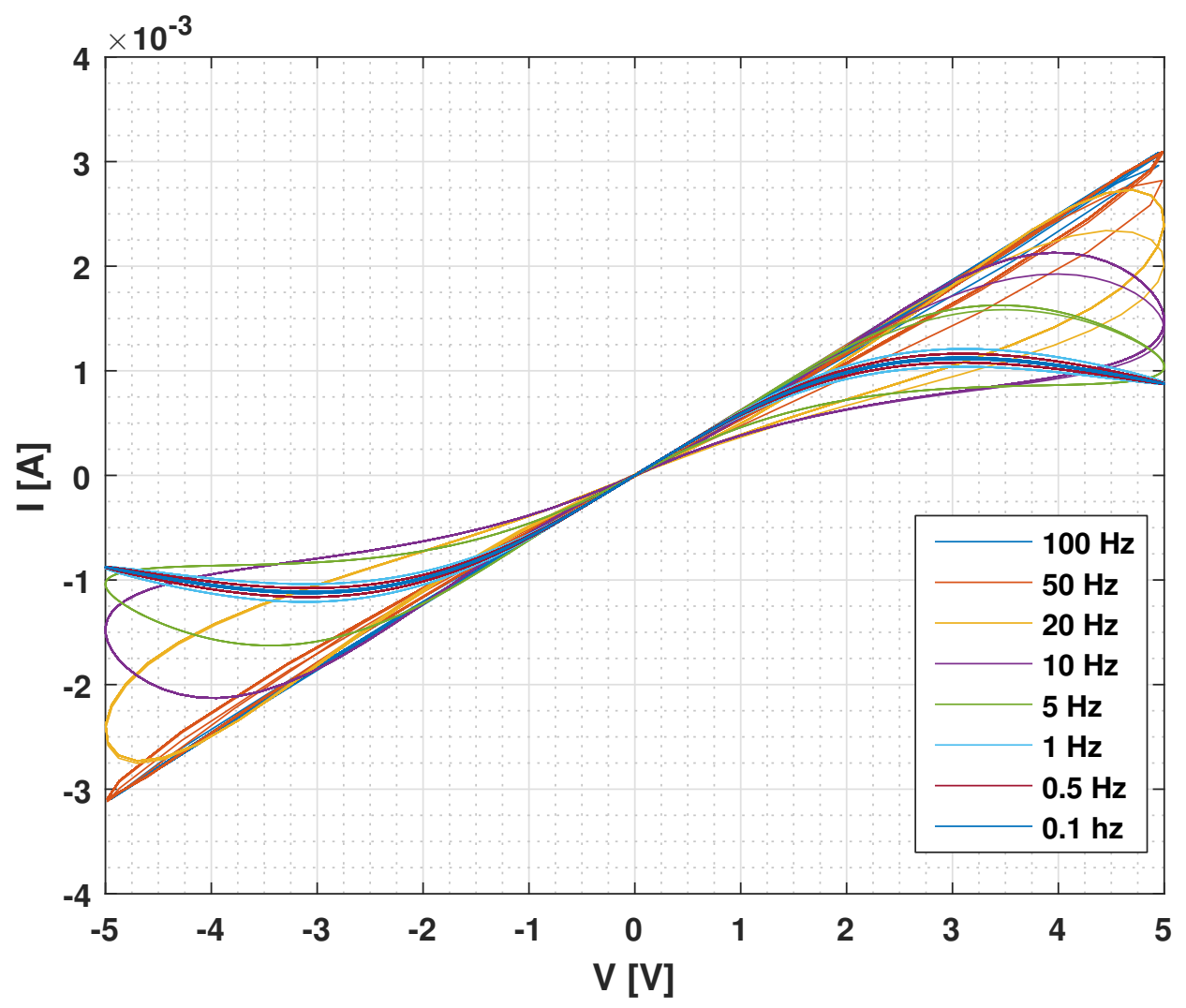

Figure 3.9: Frequency sweep results for the one-line non-linear resistor model. 


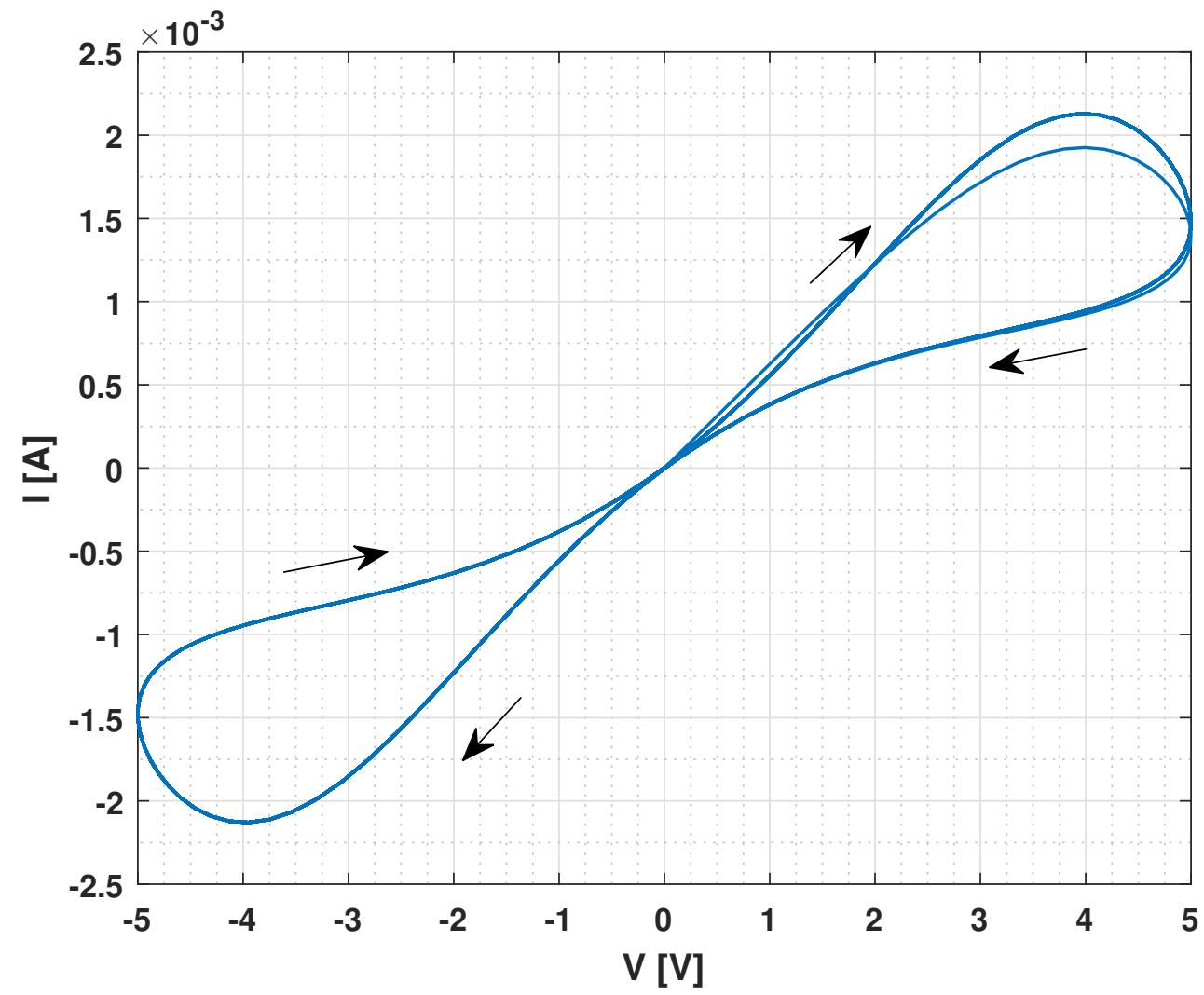

Figure 3.10: Simulation results of non-linear resistor device given a $10 \mathrm{~Hz}$ sinusoidal input of amplitude $5 \mathrm{~V}$. 
results will be compared primarily on a behavioural level.

For comparison, Figure 3.11 shows the measured results for a symmetrical PEDOT:PSS device with an input of a sinusoid of varying amplitudes and a frequency of $100 \mathrm{mHz}$.

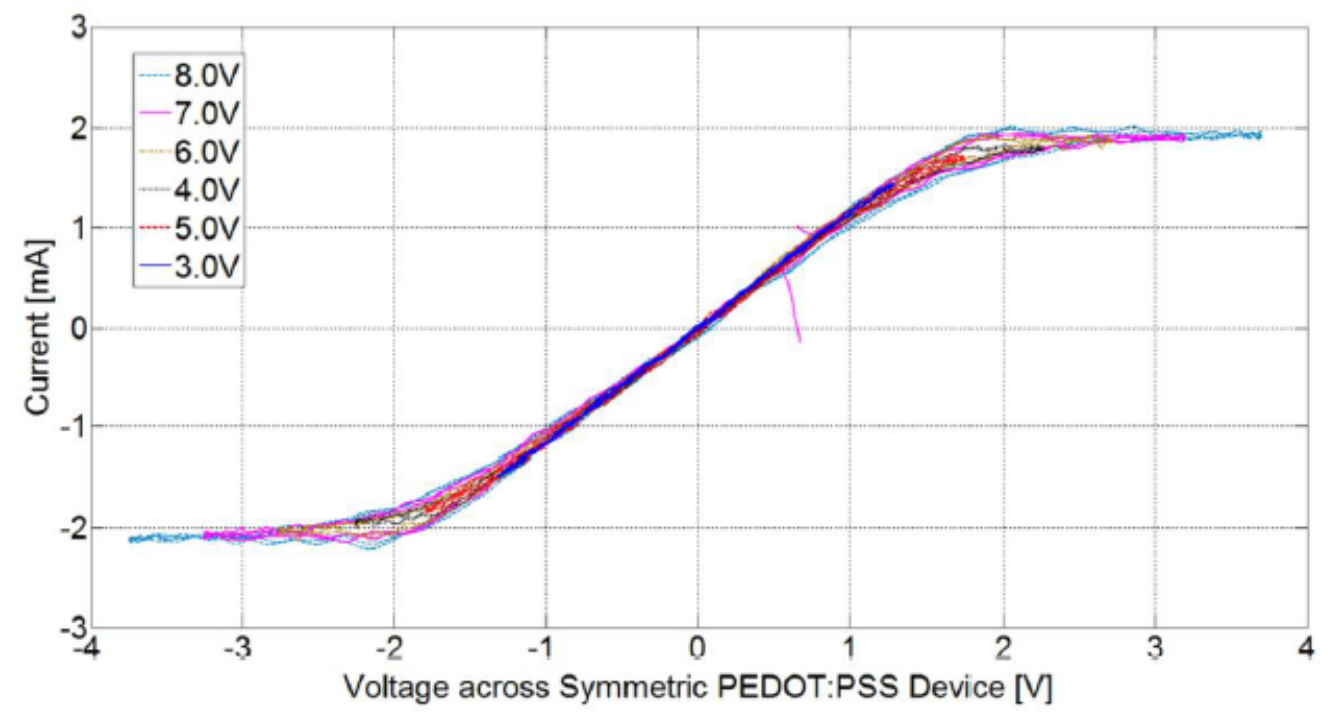

Figure 3.11: I-V curves measured across a non-linear resistor device at a frequency of $100 \mathrm{mHz}$ with varying amplitude [25].

Figure 3.12 shows the simulation results for the same type and range of input as Figure 3.11. Both figures demonstrate a linear region from approximately $-1.5 \mathrm{~V}$ to $1.5 \mathrm{~V}$, and the simulated data matches the measured data quite well. However, the simulated data clearly deviates from the measured data as the input voltage increases beyond approximately $\pm 2 \mathrm{~V}$. As it approaches total saturation, the resistance of the device should stop changing, reaching either maximum or minimum resistance. It is reasonable to conclude that the curve-fitted model does not properly account for long-term saturation or de-saturation of ions at high voltages and low frequencies. This is reasonable since the model is only partially empirical. At higher frequencies, 


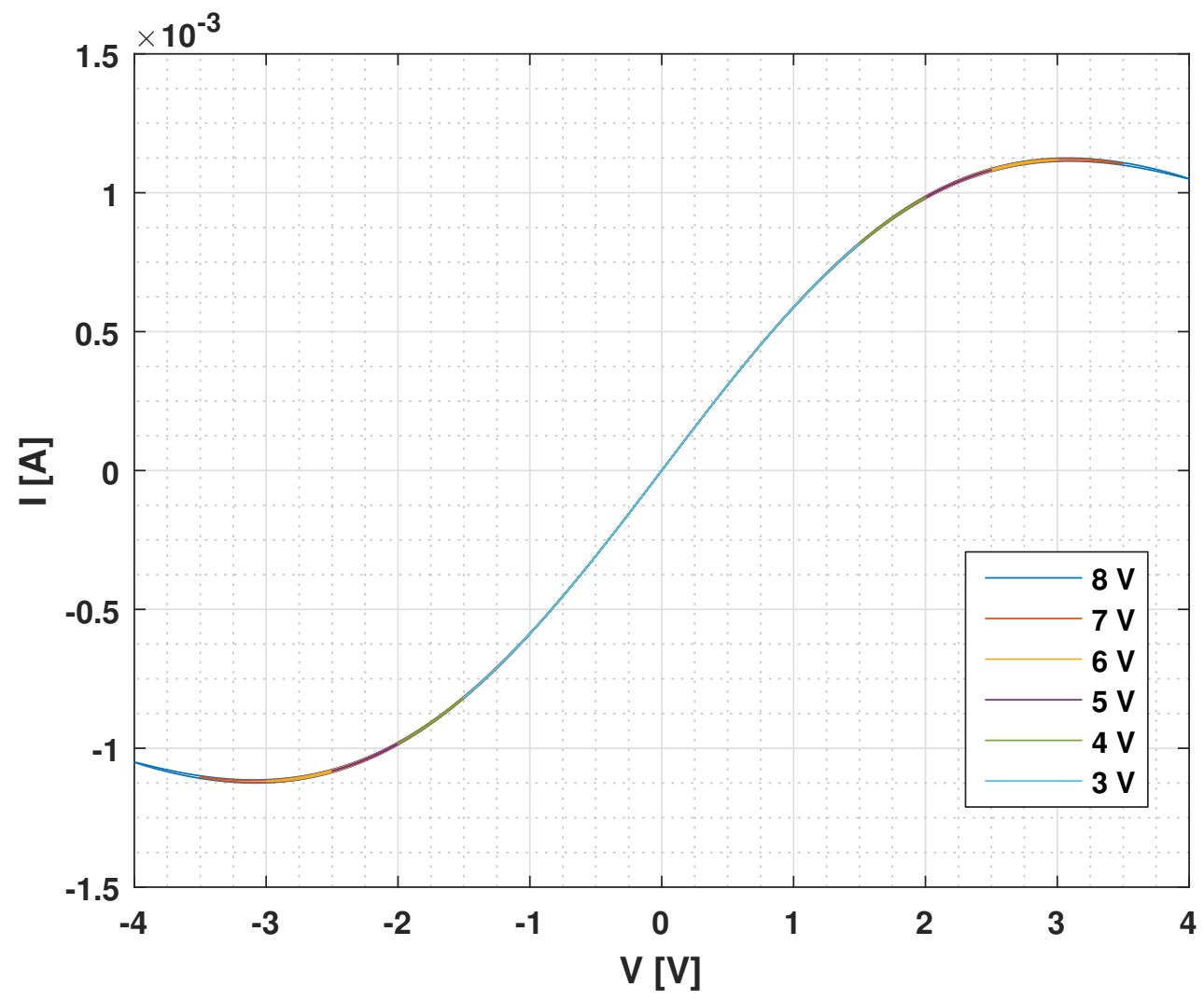

Figure 3.12: Simulation results for varying amplitudes of a sinusoidal input of frequency $100 \mathrm{mHz}$.

the model will continue to remain valid for high voltages as the device will never be completely saturated or de-saturated since the voltage changes too quickly. This can be inferred by comparing higher frequency measurements of the devices at the same amplitudes to equivalent simulations.

Figure 3.13 shows the measured data for a symmetrical non-linear resistor device across the same range of input amplitudes as previously discussed, but at a frequency of $1 \mathrm{~Hz}$. Figure 3.14 shows the simulated results for the same conditions.

From these figures, it can be seen that the simulated and measured data have very good correlation within the bounds of $\pm 3 \mathrm{~V}$. Beyond this threshold the shape of the curve remains reasonably accurate, although it again fails to account for the 


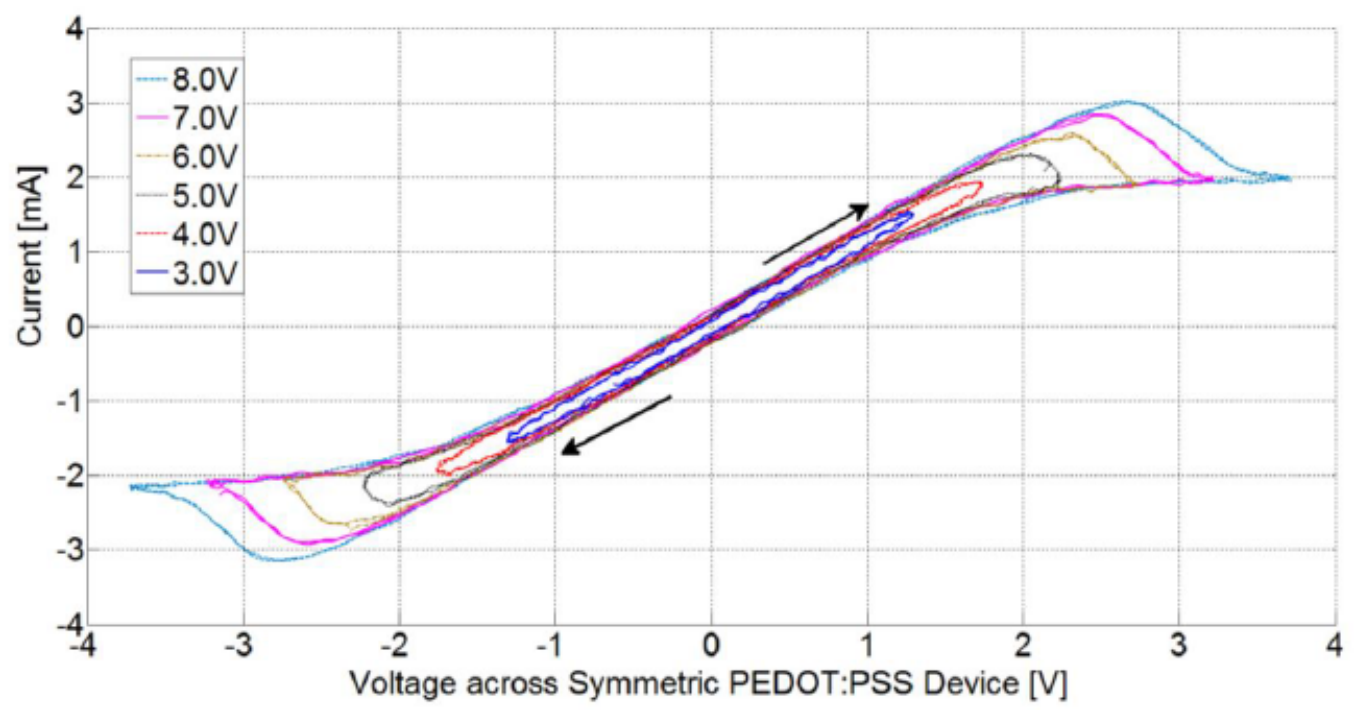

Figure 3.13: I-V curves measured across a non-linear resistor device at a frequency of $1 \mathrm{~Hz}$ with varying amplitude [25].

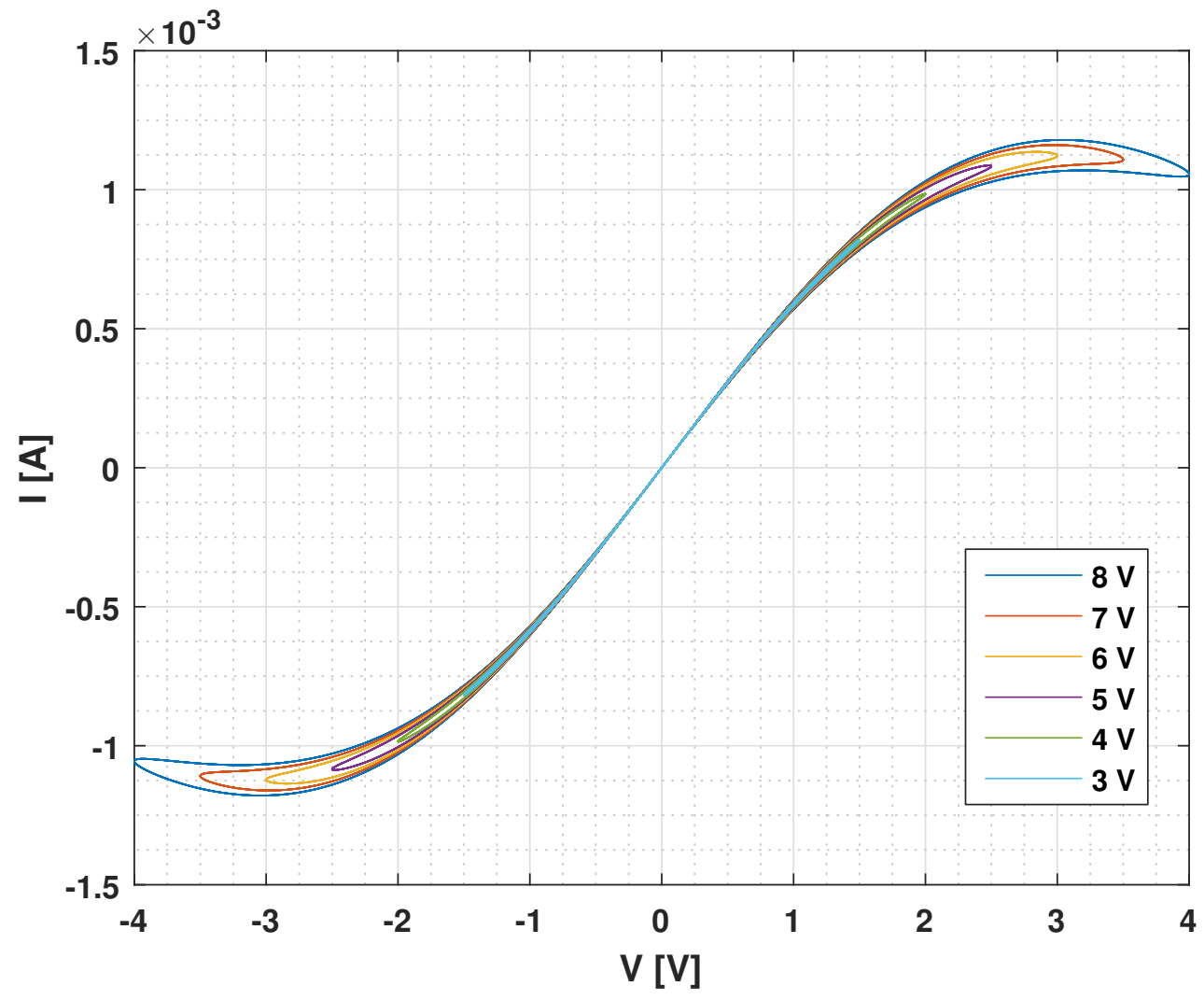

Figure 3.14: Simulation results for varying amplitudes of a sinusoidal input of frequency $1 \mathrm{~Hz}$. 
total saturation or de-saturation of the device channel. As long as the device is used within these bounds, however, the model remains accurate and provides insight into the overall behaviour of the device.

Figure 3.15 shows the measured response of a symmetric non-linear resistor for various frequency sinusoidal input signals at an amplitude of 3 V. Figure 3.16 shows the simulation results for a similar set of frequency variants at an amplitude of $5 \mathrm{~V}$. It can be seen that the curves have very similar characteristics, and that those characteristics vary in a consistent manner as the frequency is varied. These simulations are still somewhat rough estimates of idealized components, yet still provide useful insight into how the devices will behave with various input configurations. Later in this thesis, devices were developed which match the geometry in the simulations more accurately that will be compared to these simulated results again to ultimately gauge their accuracy.

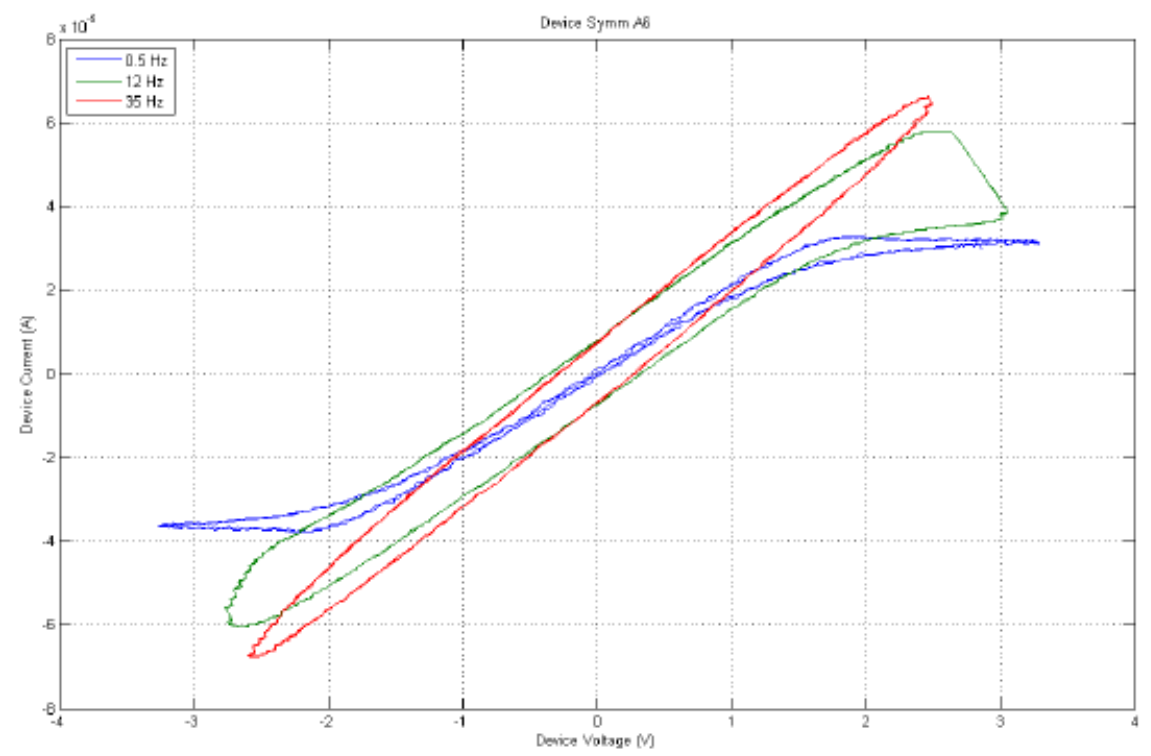

Figure 3.15: I-V curves measured across a non-linear resistor device with varying frequency and a $3 \mathrm{~V}$ amplitude [26]. 


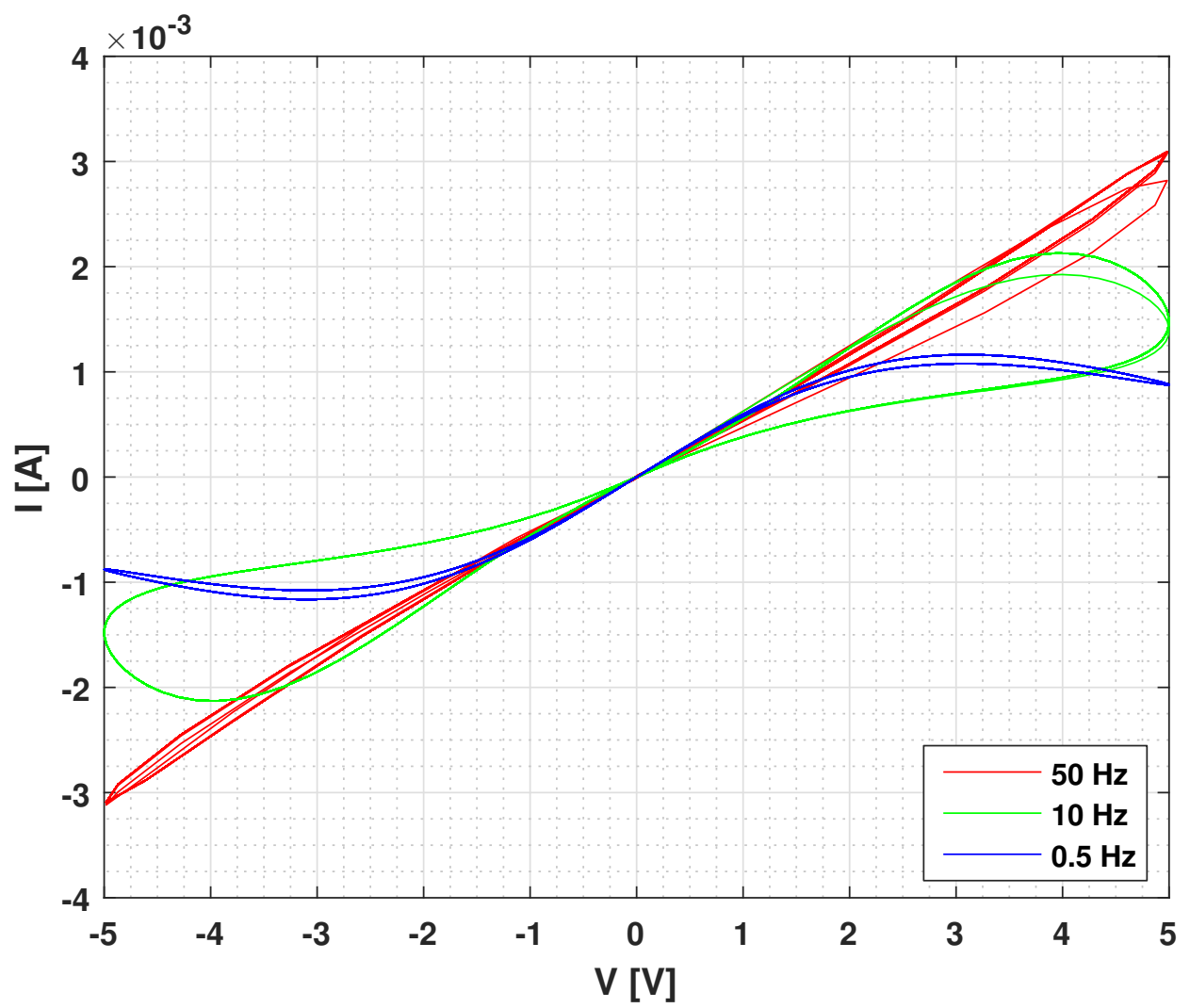

Figure 3.16: Simulation results for varying frequencies of a sinusoidal input of amplitude $5 \mathrm{~V}$. 


\section{Chapter 4}

\section{Fabrication}

The purpose of this chapter is to document the process that was used to realize the simulated device geometries from the previous chapter. First, the materials used to create the devices are described, followed by the step by step process used to fabricate a simple one-line strip of conducting polymer immersed in electrolyte solution. Next is a summary of the relevant physical parameters of the devices that were tested.

\subsection{Materials}

Previous work within this research group has focused on using PEDOT:PSS in an ink form that is suitable for screen-printing [25]. This is typically screen-printed onto a glass substrate. While accurate and versatile, this process requires the use of masks in order to be implemented. This requires time spent designing a mask, a method for

creating a custom mask, as well as a screen-printing jig that allows for alignment of multiple layers. Masks were created by using a UV laser to precisely cure emulsion that had been deposited onto a metal wire screen.

In this thesis a different method was chosen in order to accelerate the process and potentially skip these tedious and possibly expensive steps altogether. The solution 
was to simply purchase PEDOT:PSS that had already been coated onto a polyethylene terephthalate (PET) substrate, maintaining both the flexible and transparent nature of the PEDOT:PSS. Since the substrate is a polymeric plastic, it is possible to simply cut out a section of material from the large roll and use that as the active device channel.

The material used for this was Orgacon EL-350/175 [27]. This provides an even layer of PEDOT:PSS on $175 \mu \mathrm{m}$ PET base with a resistance of $350 \Omega /$ sq. The silver ink used to connect the contact pins to the conductive polymer was Dupont 5028 silver conductor [28]. In order to give the device structure, and to isolate the connection pins and silver ink from the electrolyte solution (due to corrosive effects resulting from their interaction), two different UV curable epoxies were used. One was low viscosity and was primarily used for connecting parts; the second was highly viscous and was used to form device structures. The epoxies used were Norland Optical Adhesive 81 and 68, respectively $[29,30]$. The electrolyte used for this work was previously mixed lithium perchlorate $\left(\mathrm{LiClO}_{4}\right)$ in propylene carbonate with a concentration of $1.0 \mathrm{M}$.

\subsection{Fabrication Steps}

In order to be functional, the devices must be a single strip of conductive polymer connected to usable electrodes, with free access to a reservoir of electrolyte material. In order to achieve this, a standard pin header of four pins was used, and the two pins in the middle were removed. This header is then affixed to a small glass substrate using the NOA 81 UV epoxy such that the remaining two pins extend out into the middle of the glass. Next, a strip of PEDOT:PSS-coated PET of appropriate dimensions is placed between the pins and the glass, such that the length of the strip connects the two pins together. A cross-section of this step can be seen in Figure 4.1. 


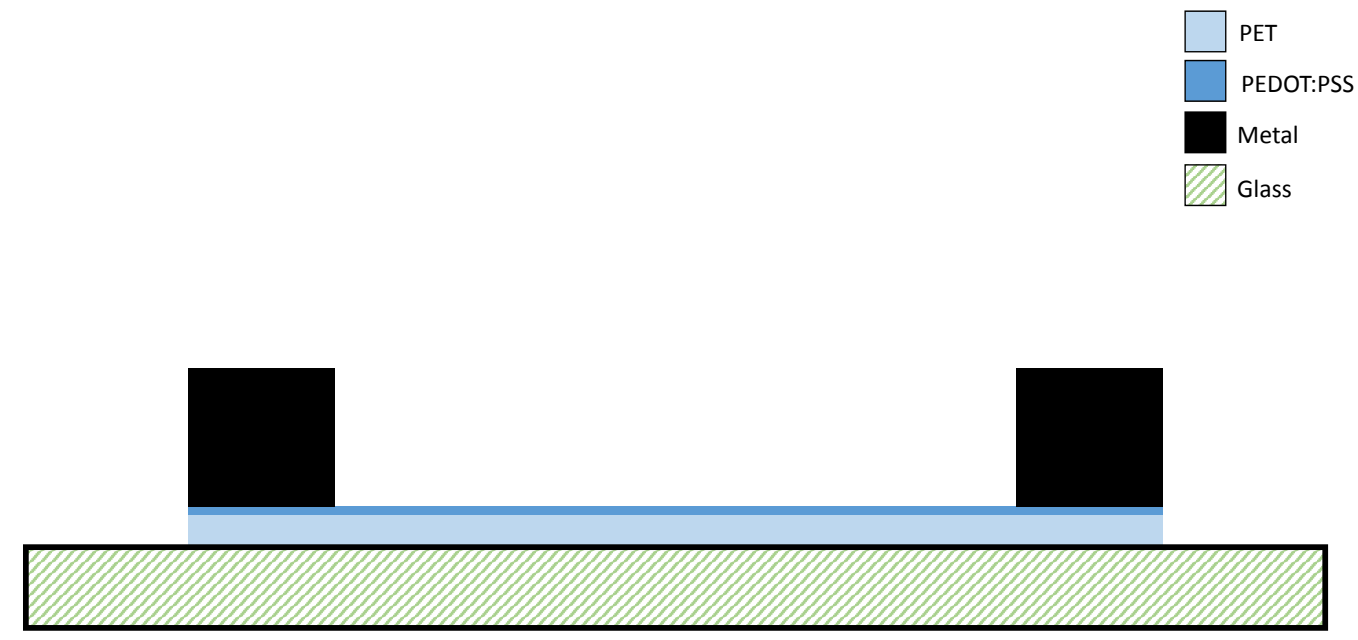

Figure 4.1: Cross-section of PEDOT:PSS on PET on a glass substrate with header pins as contacts.

The next step is to apply silver ink to the pins of the header such that they form a reliable electrical connection to the PEDOT:PSS channel. The entire device is then placed in an oven at $140{ }^{\circ} \mathrm{C}$ for approximately 6 minutes. This cures the silver ink so that it becomes solid while leaving the PEDOT:PSS unaffected due to the relatively low temperature. A cross section at this point in the process can be seen in Figure 4.2 .
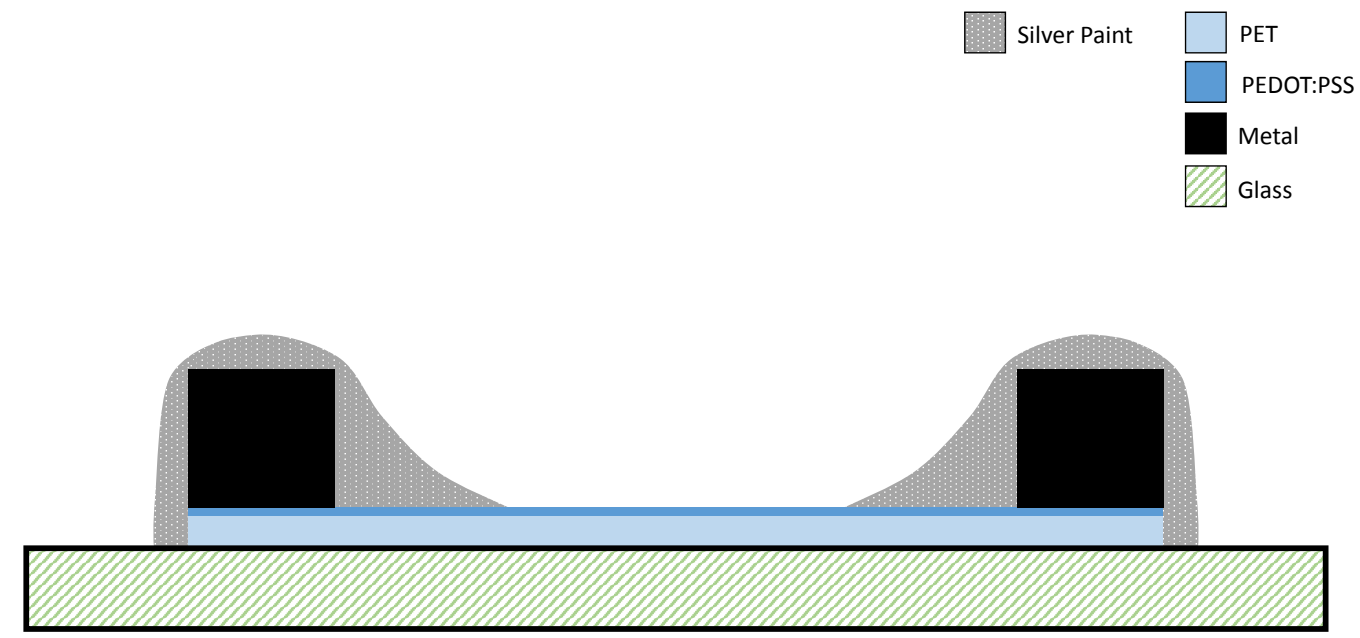

Figure 4.2: Cross-section of device in fabrication with silver ink applied to form reliable contact from pin to polymer. 
After forming a continuous conductive path from pin to pin on the header, the next step is to insulate the pins and the silver to prevent them from contacting the electrolyte while simultaneously creating a raised ring of NOA 68 UV epoxy that creates a well to contain the electrolyte and keep it in contact with the device channel. The more viscous epoxy allows for some degree of shaping of the device well. The results of this step are shown in Figure 4.3. The epoxy is also transparent so it is cured throughout with the application of an intense UV light source. While the light source cures the epoxy most of the way, in order to form a lasting chemical bond with the glass, the epoxy must be left to cure in the open air for a week or placed into an oven at $50{ }^{\circ} \mathrm{C}$ for 24 hours [30].
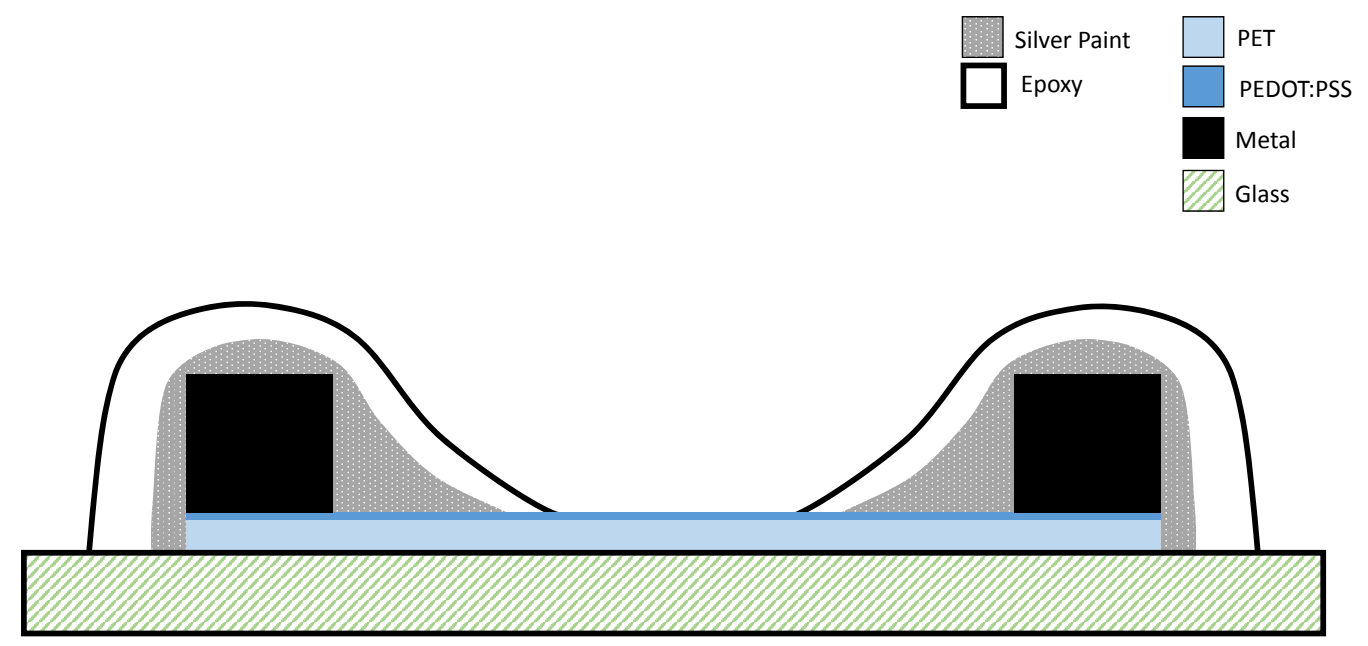

Figure 4.3: Cross-section of device in fabrication where cured UV epoxy has been applied, insulating the contacts and forming the electrolyte well.

The final step is to deposit the electrolyte solution into the device well using a syringe, completing the device structure. Optionally, another layer of NOA 68 UV epoxy can be applied by depositing it on top of the electrolyte where it will remain long enough to be mostly cured by application of UV light. This allows for the electrolyte to be sealed in, creating a fairly robust device. The device can still 
function completely normally without this seal. Figures 4.4 and 4.5 demonstrate these two steps of the process, respectively. A photo of a mostly completed device (all steps except electrolyte deposition) is shown in Figure 4.6.

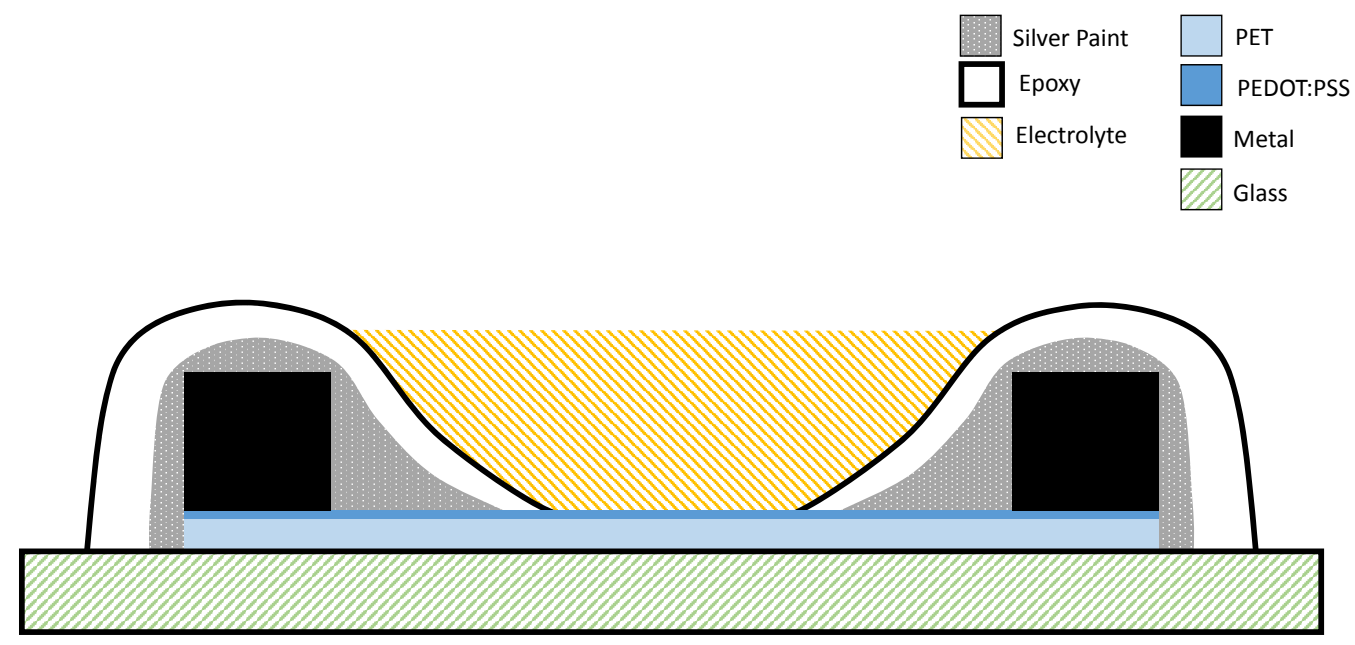

Figure 4.4: Cross-section of device in fabrication where electrolyte has been deposited into the epoxy well.
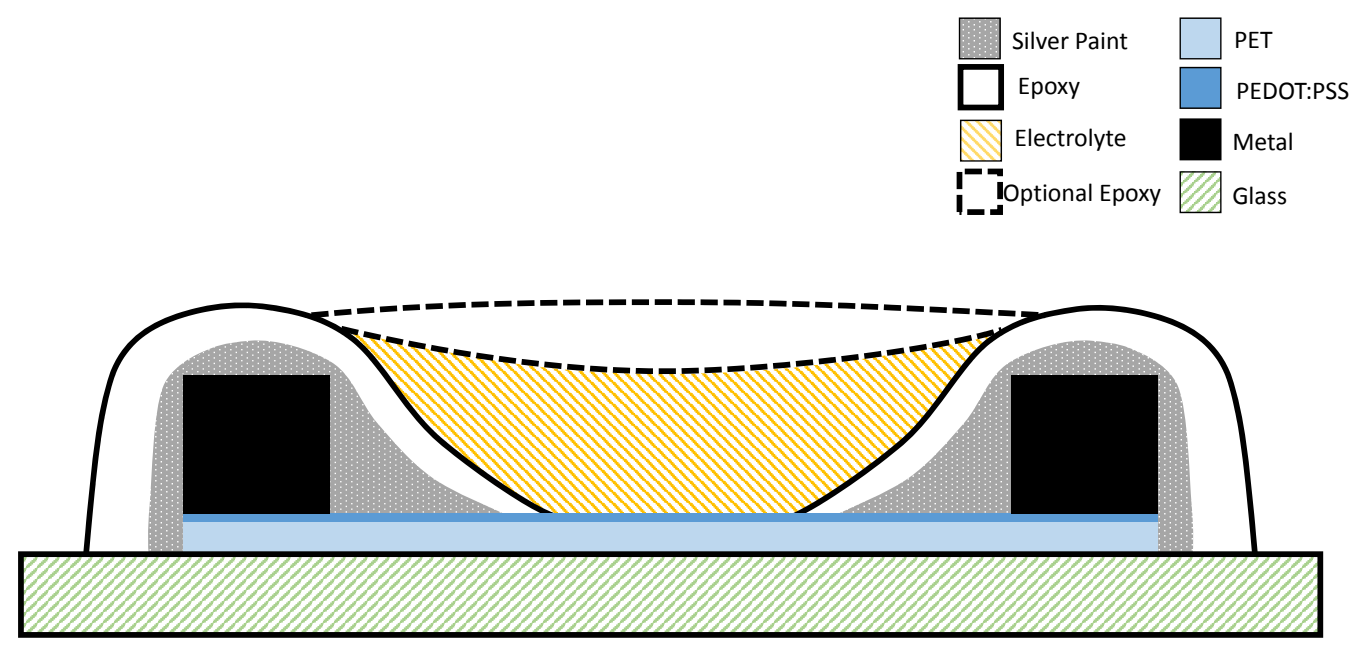

Figure 4.5: Final cross-section of device showing all applied materials including epoxy seal on top of electrolyte well. 


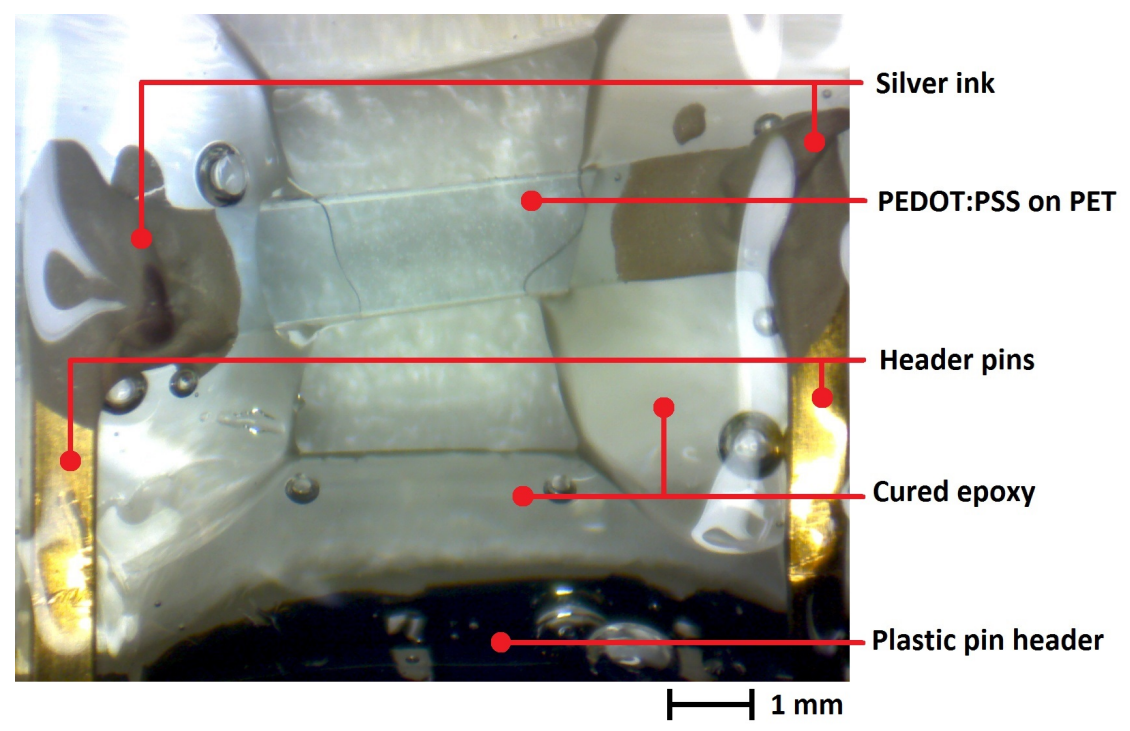

Figure 4.6: Photo of a mostly complete device. The blue strip in the middle is the PEDOT:PSS channel; the header pins and cured silver ink are visible at either end of the PEDOT:PSS strip. The epoxy that forms the device well can also be seen surrounding the device. No electrolyte has been deposited yet.

\subsection{Process Improvements}

The most significant drawback with this process is that each step for each device is carried out entirely by hand. While this allows the process to be completed in a very reasonable amount of time, it introduces a potentially infinite number of possible errors and inconsistencies that can occur. Without modifying the process too heavily, a simple jig could be created with a cutout for the device channel which could be set atop the PEDOT:PSS strip and have the epoxy deposited around it. This would allow for much more consistent device active regions and significantly reduce the time needed to build the devices. Additionally, a punch could be created to cut out PEDOT:PSS strips of a consistent size. 


\section{Chapter 5}

\section{Testing \& Results}

This chapter will describe the equipment and methods implemented in the testing of the devices previously discussed and developed. A description and evaluation of each functional device will be covered in the subsequent section. The results of the testing will then be presented and interpreted, before being compared and validated based on new simulations and previous measurements for similar devices previously created in the research group.

\subsection{Testing Setup}

The setup to test the devices was very simple. An arbitrary function generator was used to provide a sinusoidal input capable of varying both amplitude and frequency. This was connected to a series resistor, which was then connected in series with the device under test (DUT) before completing the circuit and connecting to ground. Oscilloscope probes were then attached to the source node and the node between the sensing resistor and DUT. This circuit is shown in Figure 5.1. Doing this allows for determination of the current in the circuit by dividing the difference of these two

voltages by the sensing resistance, thus enabling the creation of an I-V characteristic 
plot. The sensing resistor was chosen to be $1 \mathrm{k} \Omega$ such that it was large enough to provide a measurable amount of voltage difference as compared to the input signal, but not so large as to become a majority of the resistance in the circuit at any point in the sinusoidal cycle of the input. The measured value of the sensing resistor was $0.986 \mathrm{k} \Omega$.

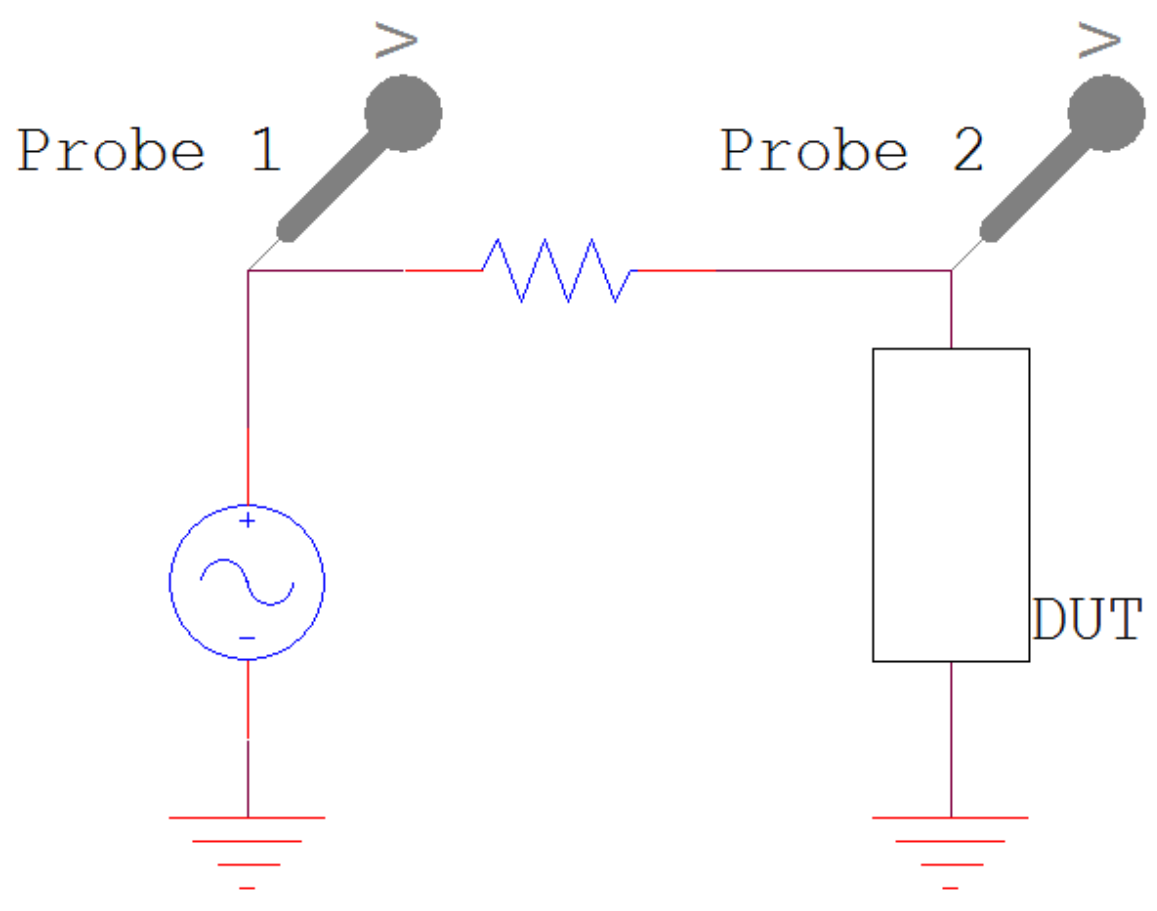

Figure 5.1: Test circuit configuration.

Table 5.1 shows a summary of the equipment used in the acquisition of the following data. 
Table 5.1: Summary of equipment used in testing.

\begin{tabular}{cc}
\hline Device & Model \\
\hline Multimeter & Tenma 72-2050 \\
Oscilloscope & Tektronix TDS 3054 \\
Voltage Probes & Tek 6139A \\
Function Generator & HP 33120A \\
Function Generator 2 & Rigol DG1022 \\
\hline
\end{tabular}

\subsection{Results}

This section will present the results of previously discussed testing methods when applied to each fabricated device that had a meaningful result. Many devices never functioned whatsoever due to issues in the fabrication process. Results of each functional device are presented, followed by a comparison to both expectations based on simulations as well as the previous tests of similar devices created in this research group.

\subsubsection{Physical Properties}

Two headers supporting five devices each were used in a breadboard to make connections and keep the devices stable while testing. The devices on each header, or "rail" were numbered from top to bottom with the pins on the left. Tables 5.2 and 5.3 contain the physical properties for the devices on rail 1 (R1) and rail $2(\mathrm{R} 2)$, respectively. Note that the devices on rail 2 were intentionally designed with a gradually increasing width as device enumeration increases in order to investigate the effects of channel width. 
Table 5.2: Physical device properties for Rail 1.

\begin{tabular}{c|ccc}
\hline Device & Width $(\mathbf{m m})$ & Length $(\mathbf{m m})$ & Dry Resistance $(\mathrm{k} \Omega)$ \\
\hline D1 & 1.34 & 8.47 & 2.90 \\
& 1.13 & & \\
\hline D2 & 1.32 & 8.75 & \\
& 1.43 & & 2.83 \\
\hline D3 & 1.50 & 7.99 & \\
& 1.58 & & 4.27 \\
\hline D4 & 1.61 & 7.85 & \\
& 1.47 & & \\
\hline D5 & 1.33 & 8.05 & \\
\hline
\end{tabular}


Table 5.3: Physical device properties for Rail 2.

\begin{tabular}{c|ccc}
\hline Device & Width $(\mathbf{m m})$ & Length $(\mathbf{m m})$ & Dry Resistance $(\mathrm{k} \Omega)$ \\
\hline D1 & 1.76 & 7.82 & 2.174 \\
& 1.91 & & \\
\hline D2 & 2.10 & 7.83 & \\
& 1.88 & & 1.270 \\
\hline D3 & 2.15 & 7.49 & \\
& 2.29 & & 1.439 \\
\hline D4 & 2.46 & 7.53 & 1.298 \\
& 2.47 & & \\
\hline D5 & 3.56 & 7.68 & \\
\hline
\end{tabular}




\subsubsection{Device Functionality}

This section contains a device-by-device presentation of results of testing as well as a brief discussion of any inconsistencies or oddities in the resulting waveforms and their potential causes. One particularly useful aspect of the devices investigated in this thesis is their predisposition to electrochromativity [31]. As ions intercalate into the PEDOT:PSS film and dedope it, the optical absorption spectrum for the material is changed [18]. This results in an apparent blue opacity property and provides the unique opportunity to determine a significant amount of information about the functionality of the device from simple visual inspection using a microscope.

This property of organic conducting polymers is used extensively to understand abnormalities in the devices fabricated for this thesis, and is highlighted in device photos by a red rectangle for this chapter. The method for observation of this effect in this thesis was to apply a constant voltage across the device using a battery pack with two $1.2 \mathrm{~V}$ batteries connected in series. The voltage was applied across the device in both directions for observation of the electrochromic properties at both ends of the device. This effect is due to the presence of positively charged ions intercalating into the material, and thus will be observed on the device terminal that is connected to the negative side of the batteries. 


\section{Device R1D1}

Device R1D1 was one of the most promising devices during initial testing. Figures 5.2 and 5.3 show a clean, straight edge at both ends of the device, resulting in a uniform cross-sectional resistance and good symmetry. It is a little difficult to see the change in opacity in Figure 5.3, however it is noticeable as a blue discolouration beneath the reflection on the left of the device when compared to the photo with no applied potential.

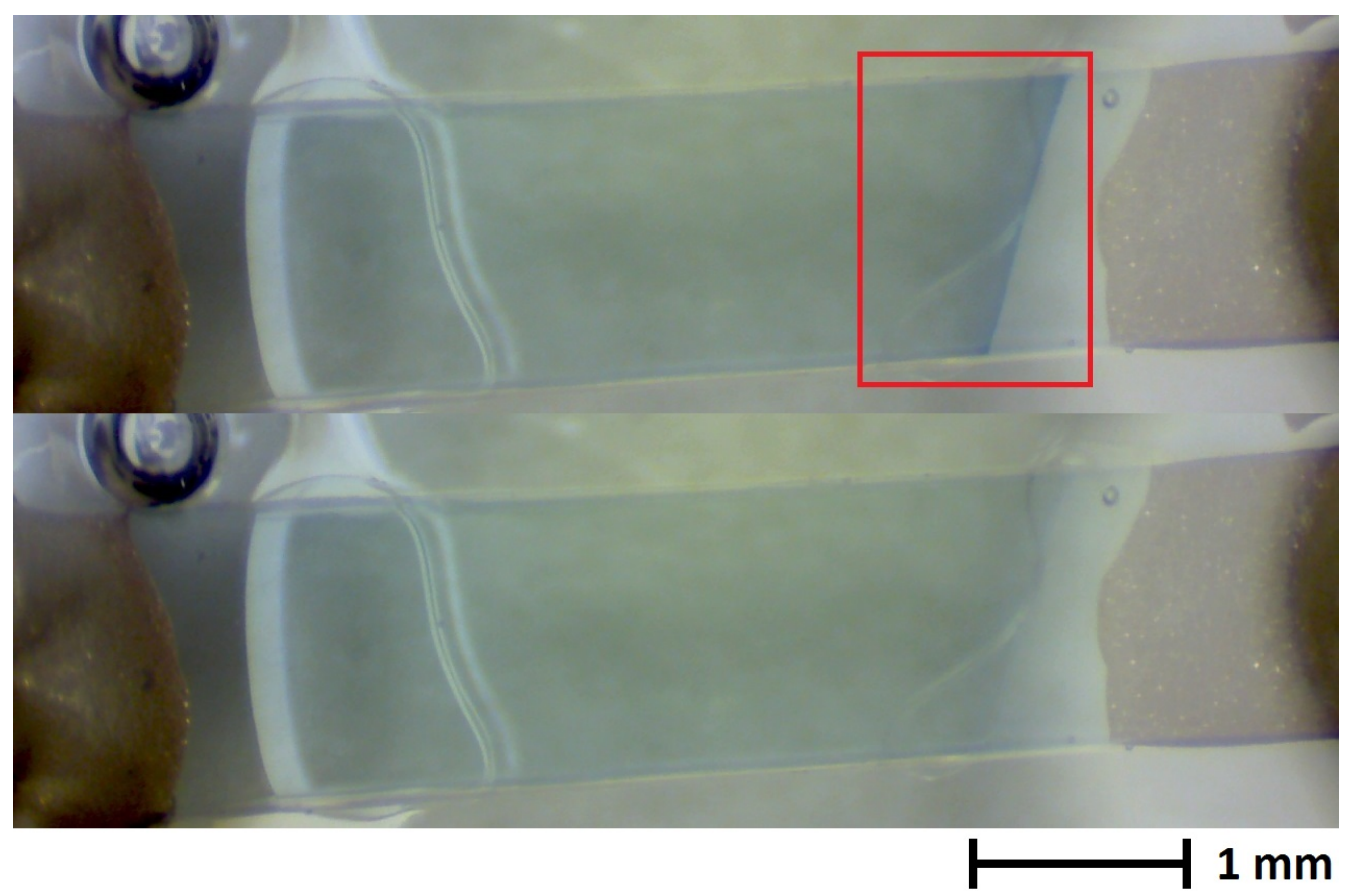

Figure 5.2: Side by side of device R1D1 with positive voltage applied to the left electrode with respect to the right electrode (top) and with no applied voltage (bottom). 
The results of this symmetry can be seen in the voltage response of the device in Figure 5.4. The same or very similar curve shape is seen on both ends of the device (i.e. at both high and low potential). Figure 5.5 shows a zero-crossing hysteresis loop with wide opening at either end. As expected, it is also highly symmetrical.

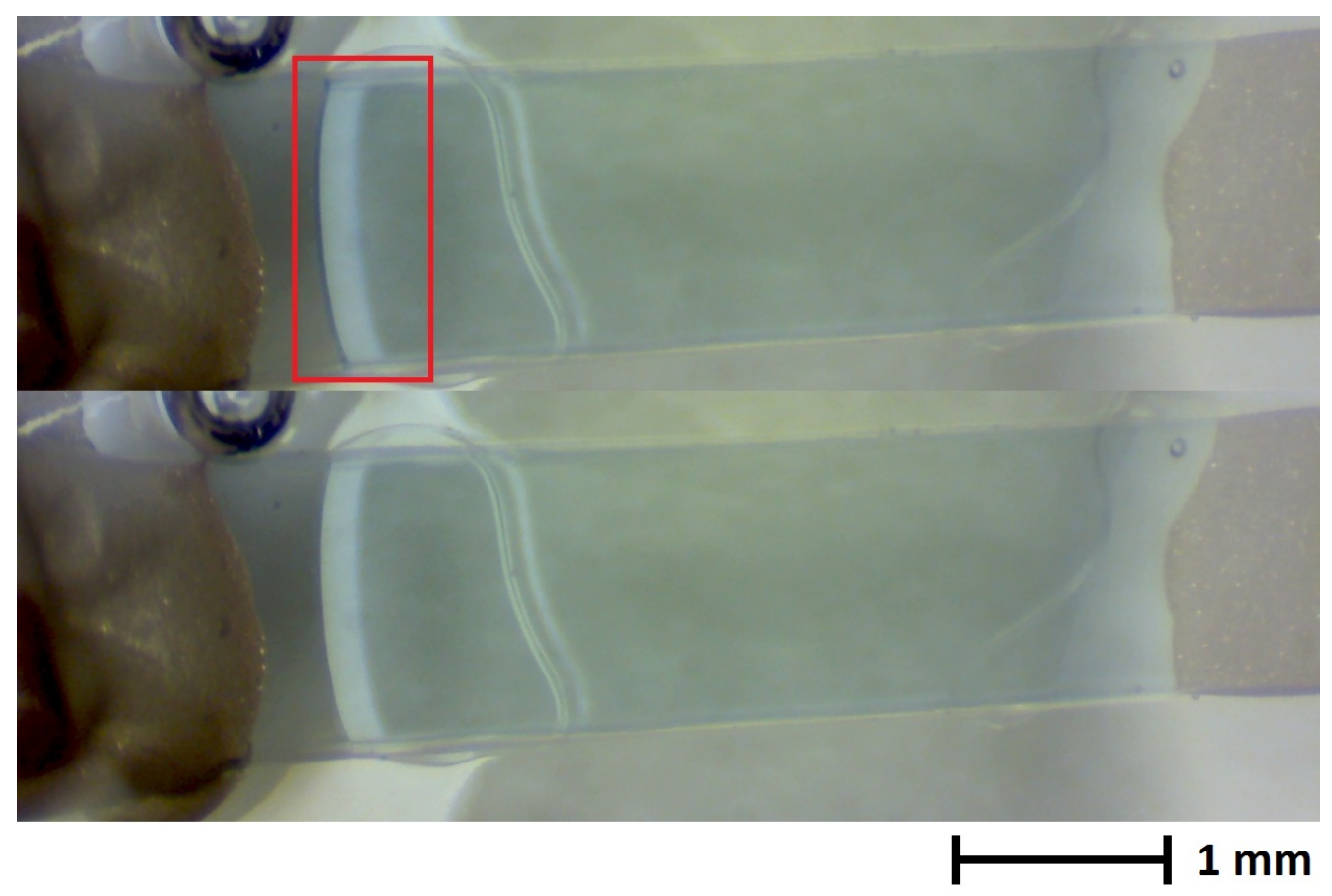

Figure 5.3: Side by side of device R1D1 with positive voltage applied to the right electrode with respect to the left electrode (top) and with no applied voltage (bottom). 


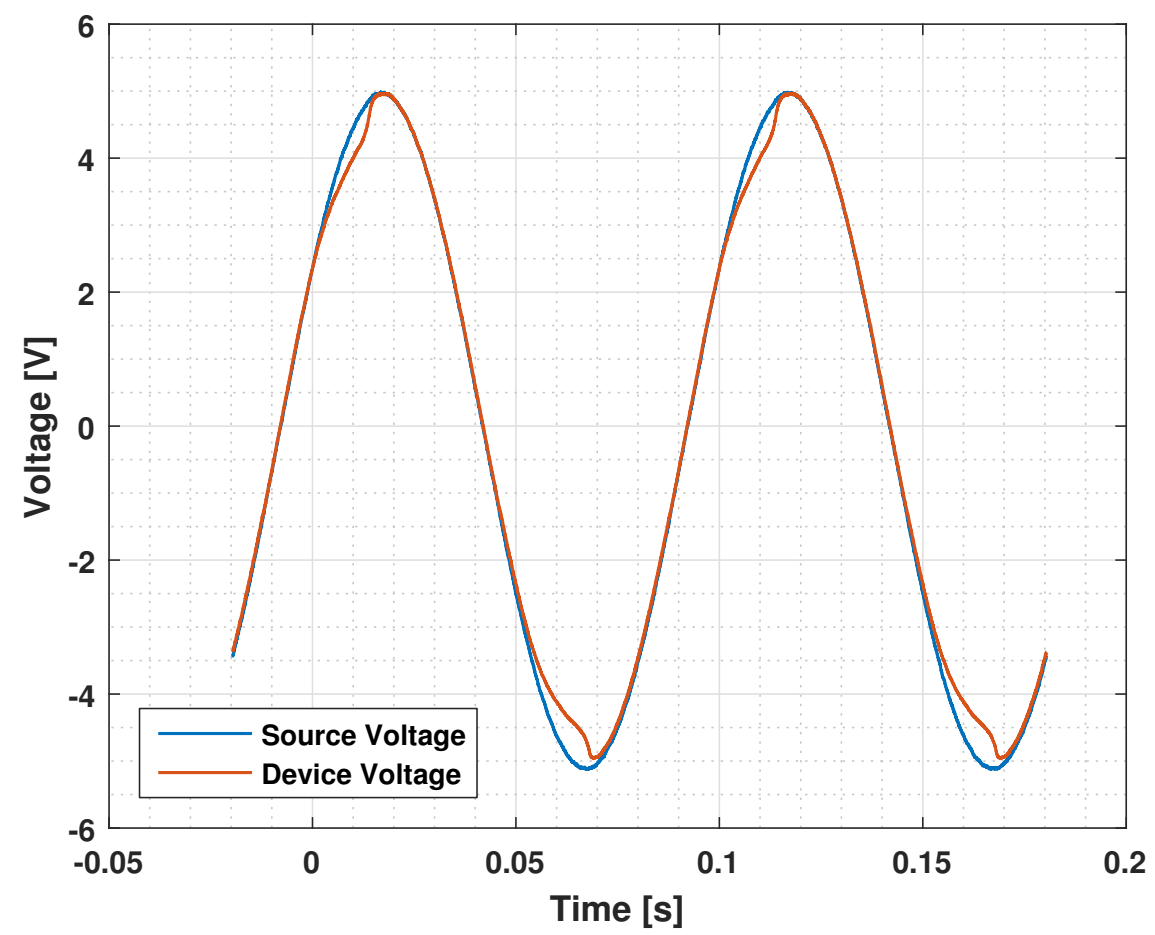

Figure 5.4: Voltage over time for device R1D1 at $10 \mathrm{~Hz}$ and $5 \mathrm{~V}$ amplitude.

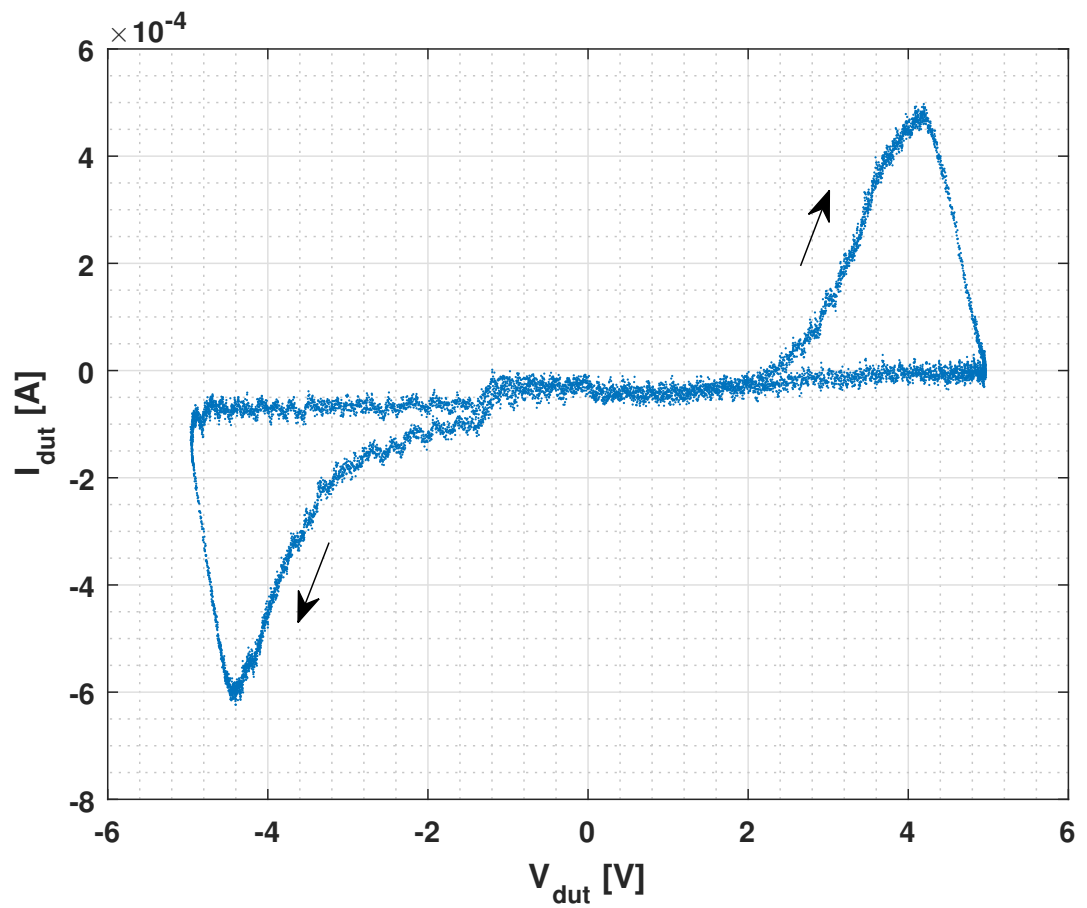

Figure 5.5: I-V curve for device R1D1 at $10 \mathrm{~Hz}$ and $5 \mathrm{~V}$ amplitude. Arrows indicate signal change with respect to time. 
The current of the device remains low until the input voltage reaches about half of its maximum value, at which point it increases rapidly. At approximately $4 \mathrm{~V}$, the resistance begins to increase and the current starts to drop off again while voltage continues to increase. As the voltage reaches its maximum value, the current has returned to being low and stays there until high negative voltage is reached, at which point the other end of the device begins to get dedoped by intercalating ions and the process repeats for the negative half-cycle. This exact behaviour is not quite the expected result, as can be seen if compared to Figure 3.10. The transitions from high resistance to low resistance are far more abrupt than predicted. This is most likely due to the relatively high voltage input of the device. In the past, it has been demonstrated that voltages beyond $2 \mathrm{~V}$ can cause reactions in the polymer other than the desired and reversible redox reactions that can permanently affect conductivity [25]. The data in Figure 5.5 were acquired shortly after the voltage had been applied and as such it is likely that the polymer had not yet significantly degraded, but was showing first signs of the degradation. This effect is the likely cause of the close match between the two voltages measured. The degradation would cause a much higher resistance on the device channel and result in a much smaller differential voltage detected across the sensing resistor. Shortly after these data were collected, the device ceased conducting entirely, appearing for all intents and purposes as an open circuit. Later visual inspection of the device using a microscope yielded no discernible visible changes. 


\section{Device R1D2}

Figures 5.6 and 5.7 indicate device R1D2 to be working well, visually. The dedoped region is quite large for both ends of the device, as well as reasonably even across the device. The large mark on the left side of the device is an area where some of the UV epoxy ran underneath the device before being cured, and does not affect the operation of the device.

Figure 5.6 shows device R1D2 with a DC potential applied across the device from its left side (positive) to its right side (negative), and shows a change in the colour and transparency of the material, indicating an intercalation of ions. Figure 5.7 shows the same device with the potential applied in the other direction.

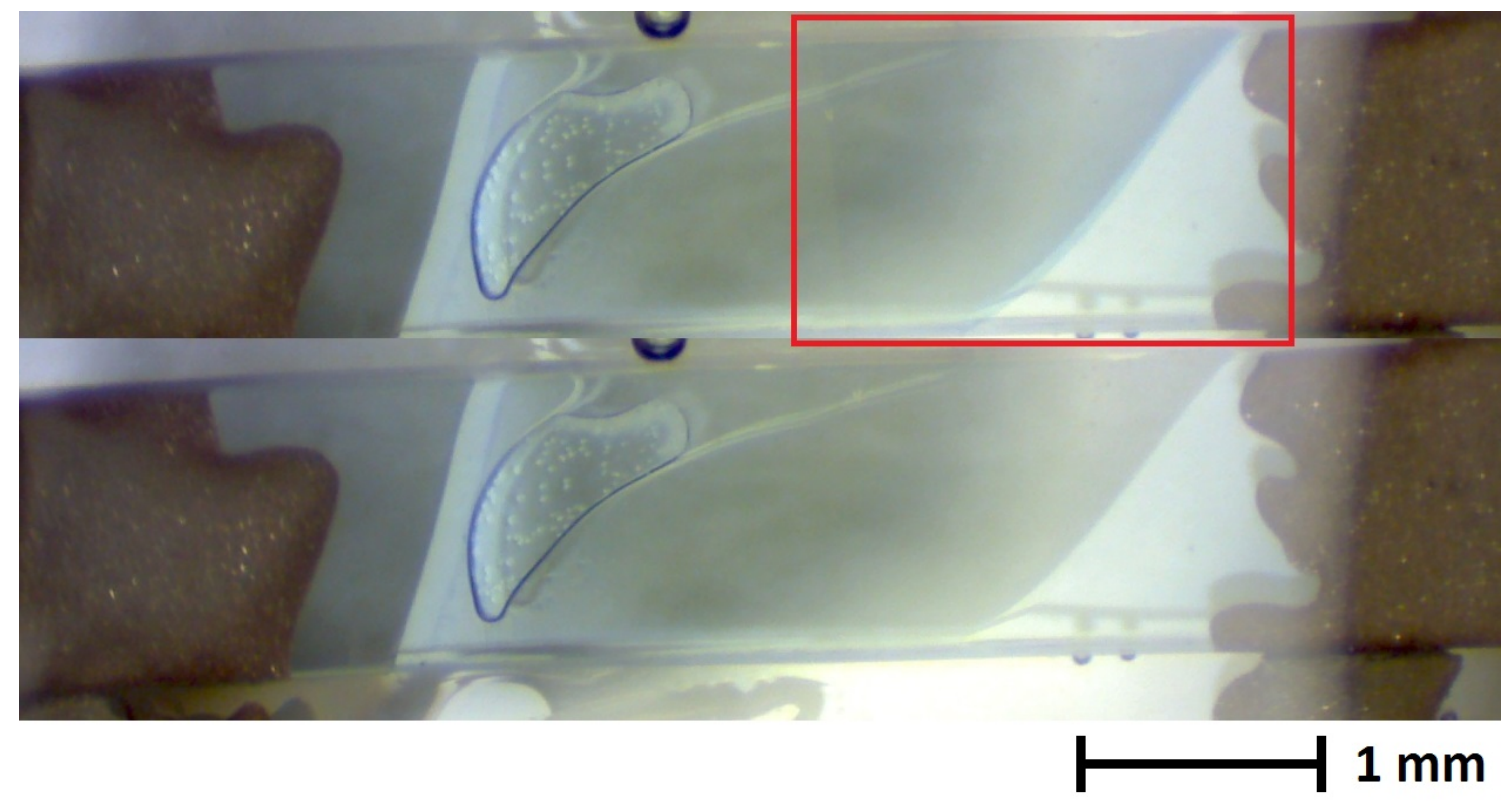

Figure 5.6: Device R1D2 with positive voltage applied to the left electrode with respect to the right electrode (top) and without (bottom). 


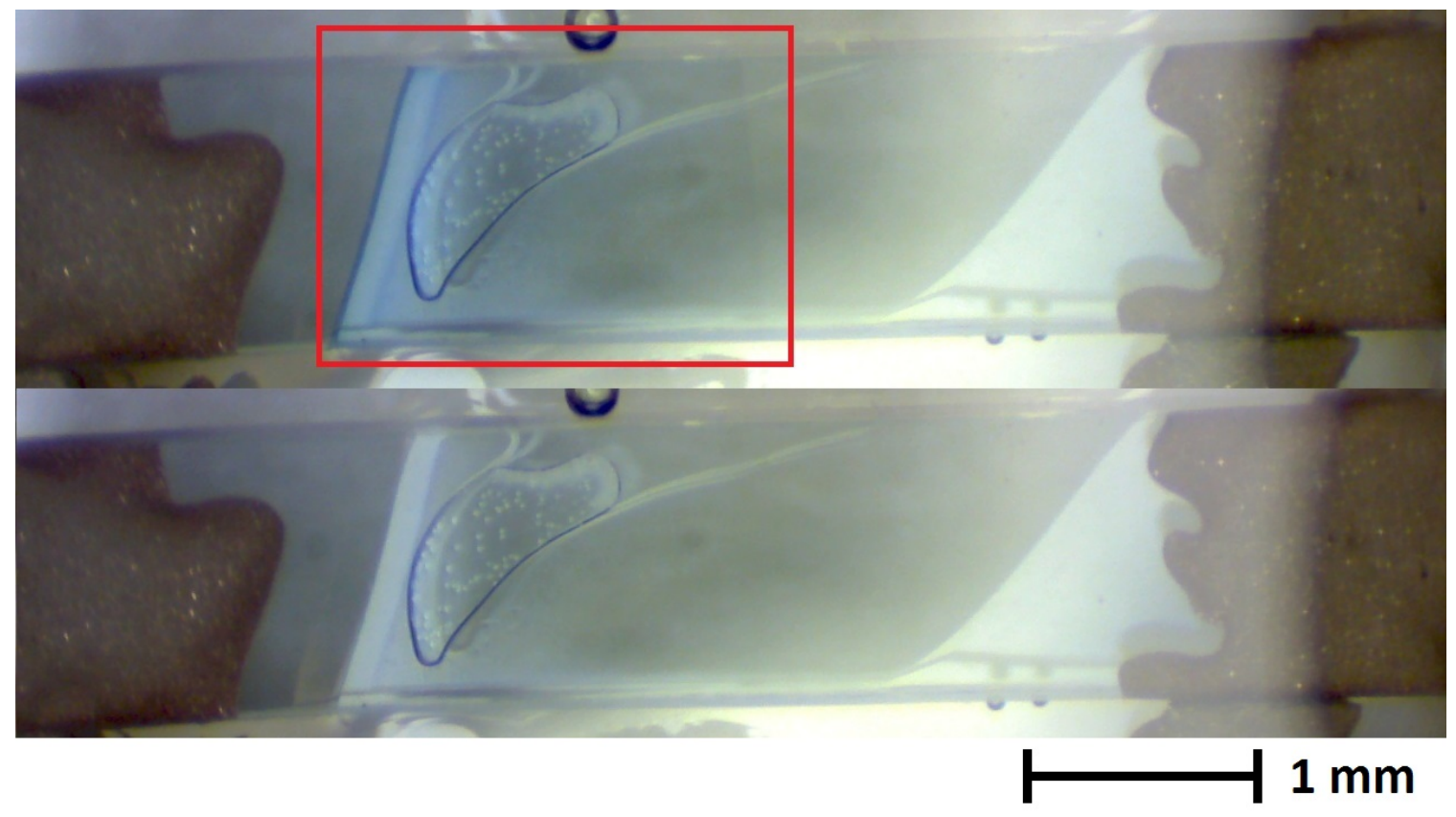

Figure 5.7: Device R1D2 with positive voltage applied to the right electrode with respect to the left electrode (top) and without (bottom).

Figure 5.8 shows the measured voltage response of the device for an AC sinusoidal input of frequency $10 \mathrm{~Hz}$ and amplitude $3 \mathrm{~V}$. Figure 5.9 shows the I-V curve for the device, exhibiting a non-crossing hysteresis characteristic, as expected. The hysteresis loop is very similar to the response measured for device R1D1, except that it never quite crosses zero. Inspecting the ion intercalation area in Figures 5.6 and 5.7 shows that it makes up the majority of the device channel. Only a very short length of the device is not dedoped. As a result, many of the $L i^{+}$ions remain in the PEDOT:PSS film perpetually, preventing the curve from reaching zero. At a lower frequency, the loop would become zero-crossing again as the additional time would allow ions to be expelled from the PEDOT:PSS. 


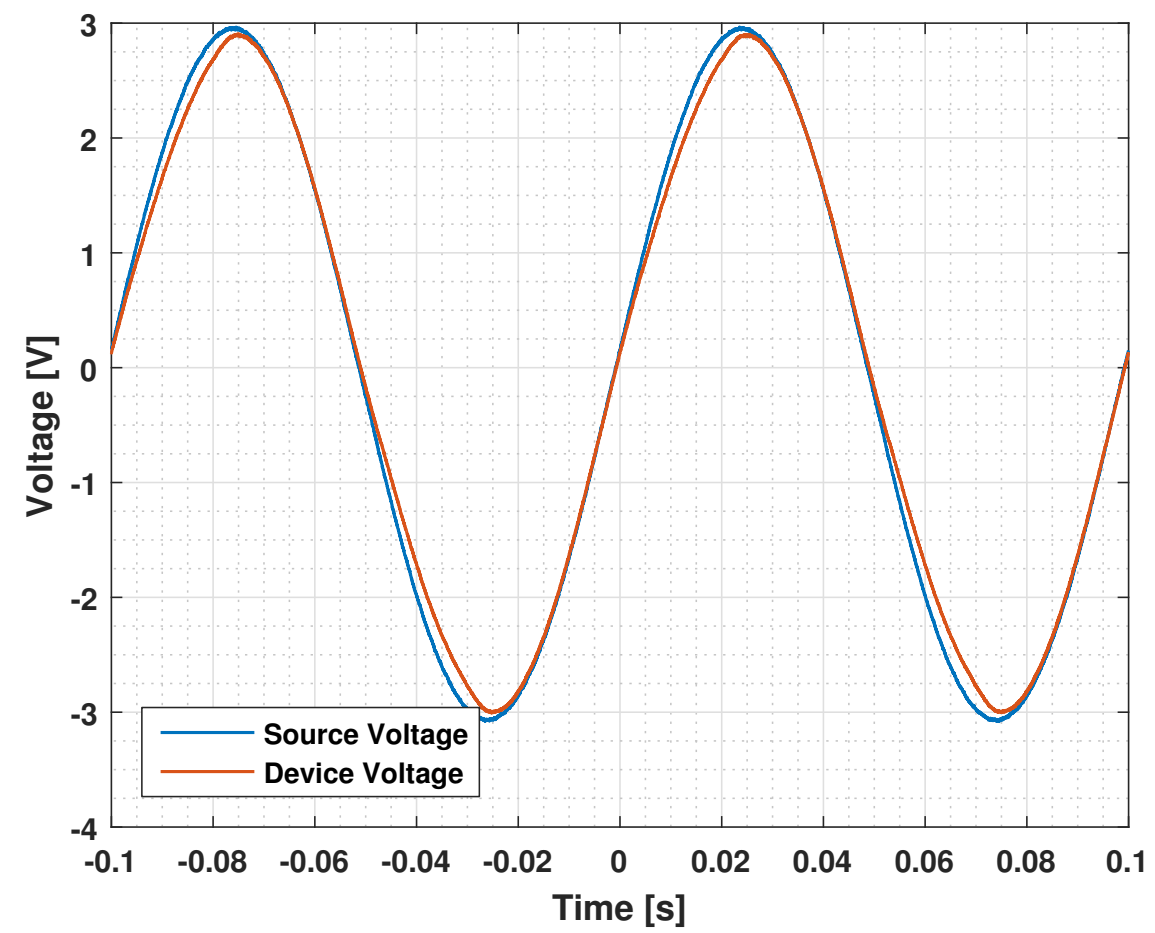

Figure 5.8: Voltage over time for testing of device R1D2 at $10 \mathrm{~Hz}, 3 \mathrm{~V}$ amplitude.

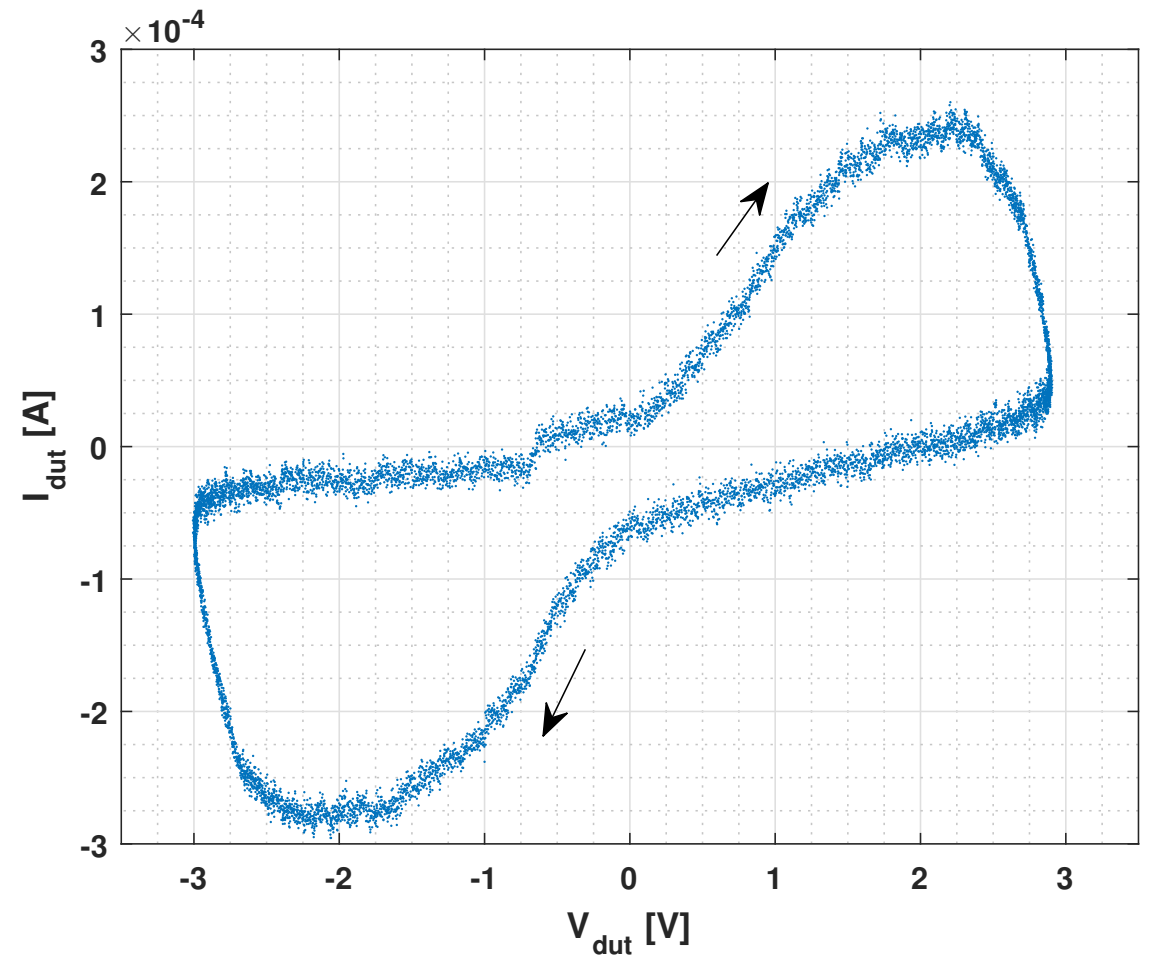

Figure 5.9: I-V curve for R1D2 at $10 \mathrm{~Hz}$. Arrows indicate signal change over time. 


\section{Device R1D4}

Device R1D4 also has a clearly defined active region with good electrode isolation from the electrolyte. Figures 5.10 and 5.11 show these properties. Note that the left side of the device is somewhat close to the electrode, as can be seen in 5.11.

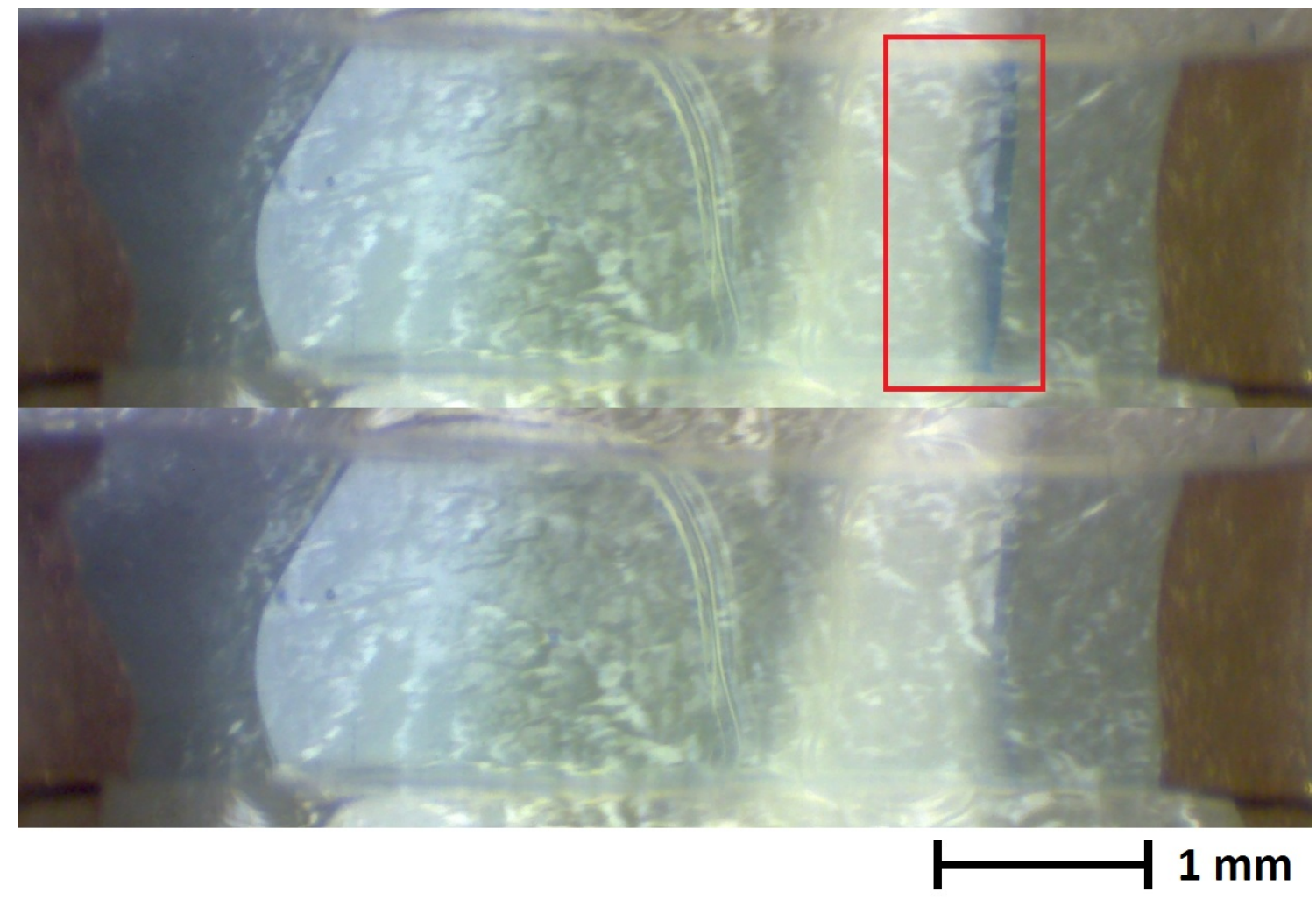

Figure 5.10: Device R1D4 with positive voltage applied to the left electrode with respect to the right electrode (top) and without (bottom).

Figure 5.12 shows the voltage change on the device over time, where it can be seen that the signal is not perfectly symmetrical. Looking at the I-V curve in Figure 5.13 better demonstrates this inconsistency. A possible cause of the sudden changes in current at high voltage could be due to intercalated $\mathrm{Li}^{+}$ions migrating through the PEDOT:PSS layer and reaching the electrode due to the shorter distance between the electrode and the electrolyte. The issue is almost assuredly due to the geometry of the device, as no similar inconsistency can be seen on the other side of the hysteresis 
loop since the epoxy barrier separating the electrode from the electrolyte is greater.

The response remains a valid non-self-crossing response for a non-linear resistor device. Once again, the slow rate of ion movement prevents the loop from crossing through zero.

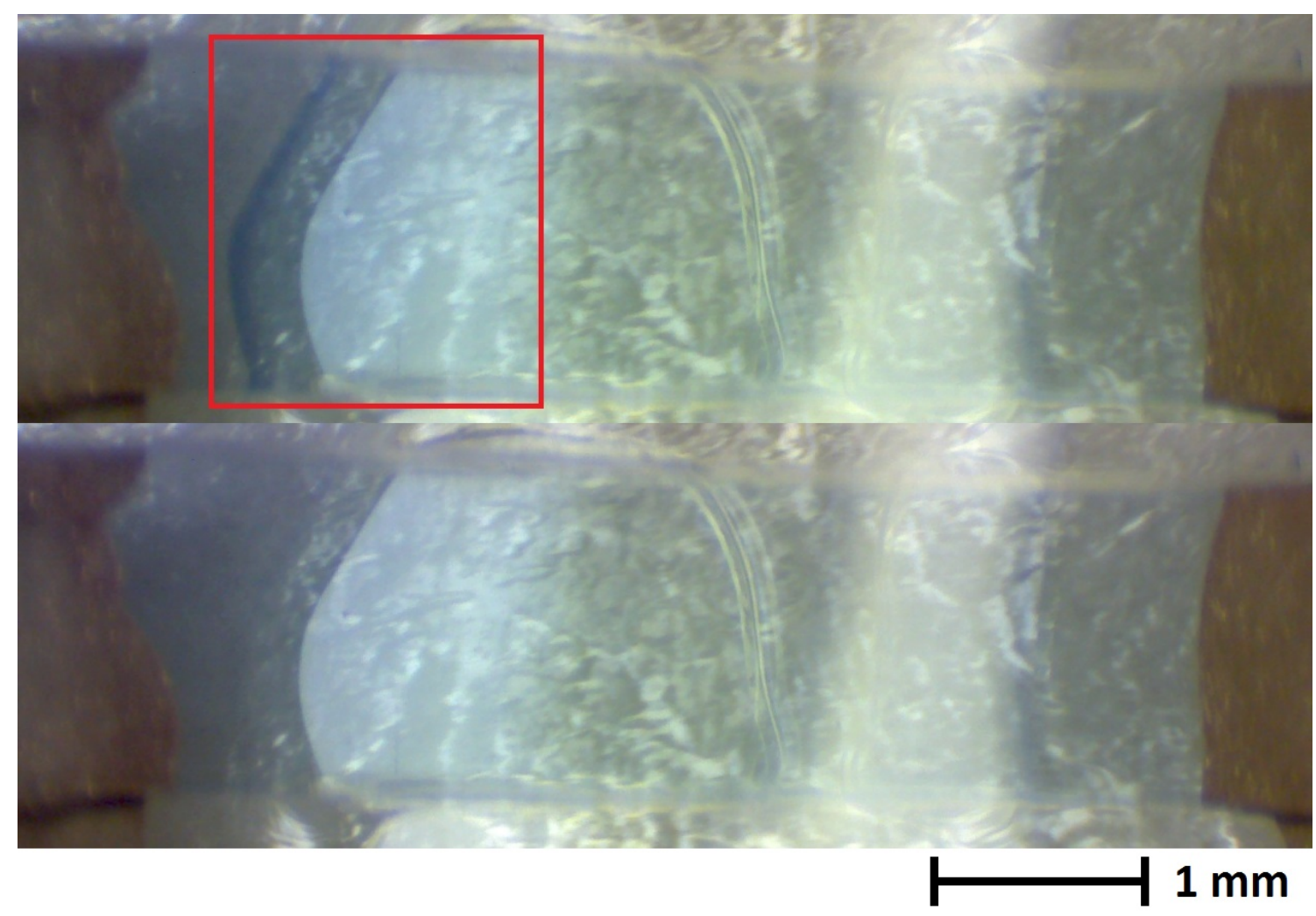

Figure 5.11: Device R1D4 with positive voltage applied to the right electrode with respect to the left electrode (top) and without (bottom). 


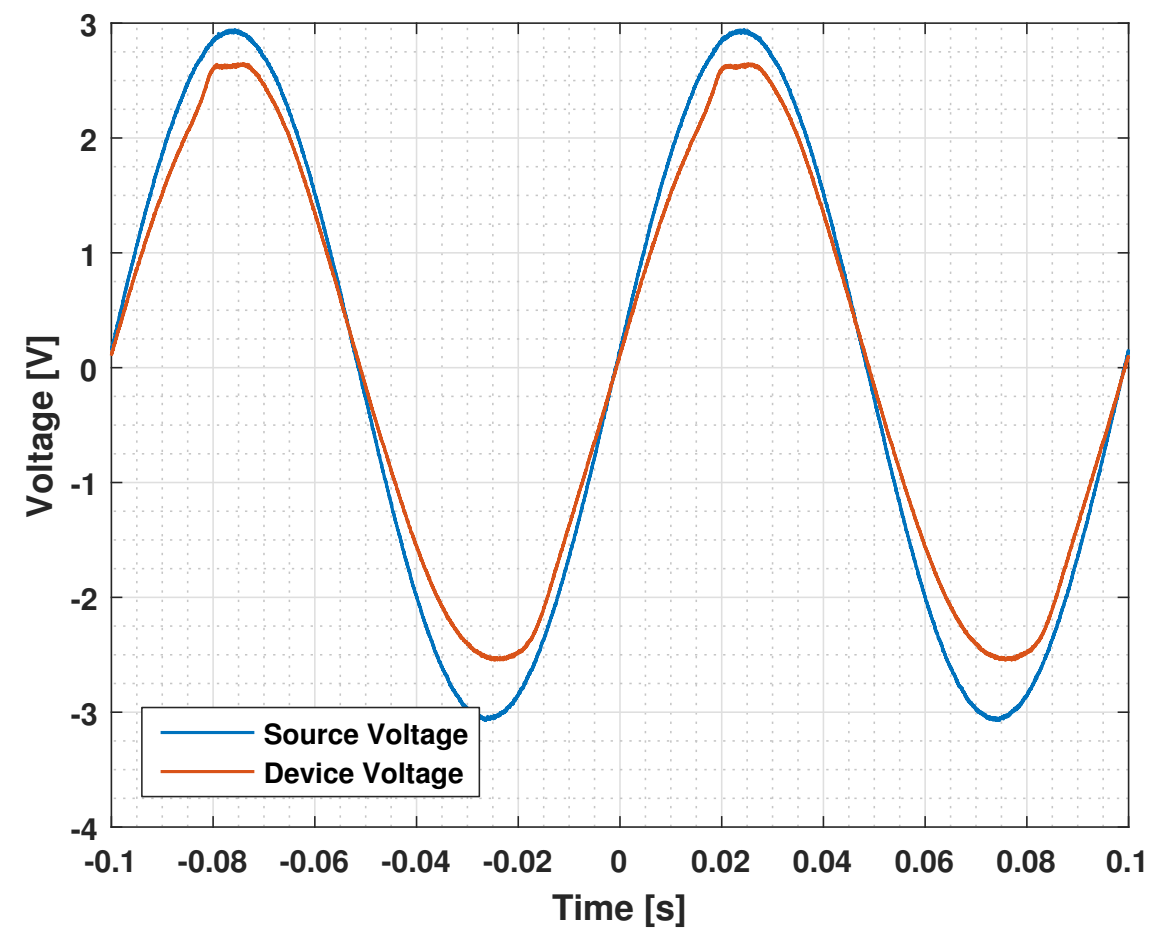

Figure 5.12: Voltage over time for testing of device R1D4 at $10 \mathrm{~Hz}, 3 \mathrm{~V}$ amplitude.

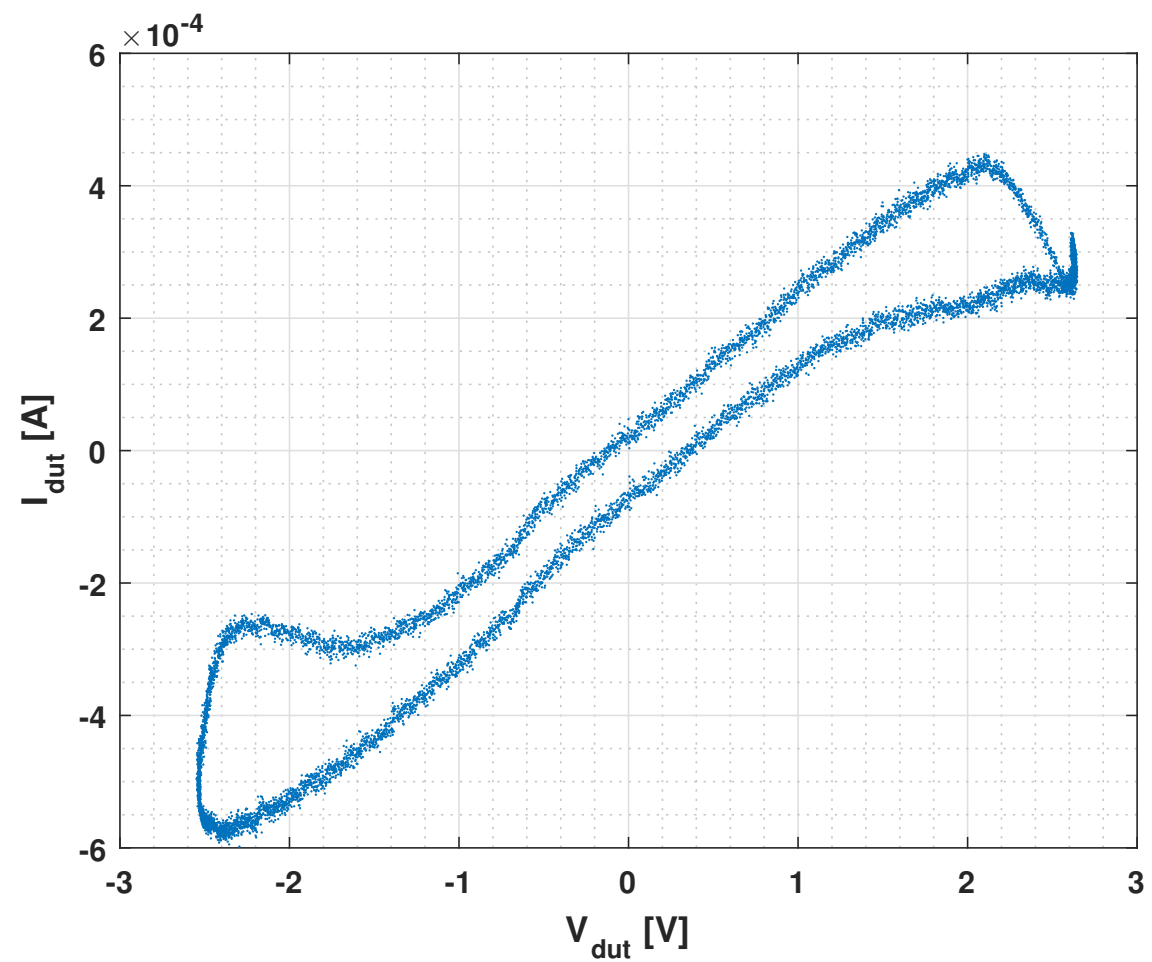

Figure 5.13: I-V curve for device R1D4 at $10 \mathrm{~Hz}$. 


\section{Device R1D5}

It is clear from Figure 5.14 that device R1D5 will not work in the expected manner. The opacity and colour change caused by intercalated ions indicate that there is a direct connection from the right electrode to the electrolyte solution, despite the majority of that end of the device being acceptable. Figure 5.15 indicates that the other end of the device should function normally as expected. While not an ideal device, it can still serve as an interesting case study for unexpected effects or possibly even identifying future manufacturing defects.

The effect of this fabrication error can be seen clearly in both Figures 5.16 and 5.17. In Figure 5.16, at the positive end of the sinusoid, the non-linearity inherent in the system is evident, whereas as at the negative end there is no such non-linearity, only a voltage drop as though there is a series resistance. This is further demonstrated in Figure 5.17 where the classic open hysteresis loop that can be expected from these devices is seen, but only on the positive half of the sinusoid input. For the negative half, it functions as a basic resistor. Looking closely, there is a very minor non-linearity in the form of a very narrow hysteresis loop, but it is narrow enough to approximately be just a constant resistance. This correlates with the fabrication error. The devices on R1 were connected to the test circuit (Figure 5.1) such that the right electrode was connected to the voltage source, and the left electrode to ground. Ions intercalate on the side of the device that is at the lower resistance. So then it would be reasonable that the negative half of the cycle would be the one affected by the fabrication error, since that's the half of the cycle where ions would intercalate in that region. 


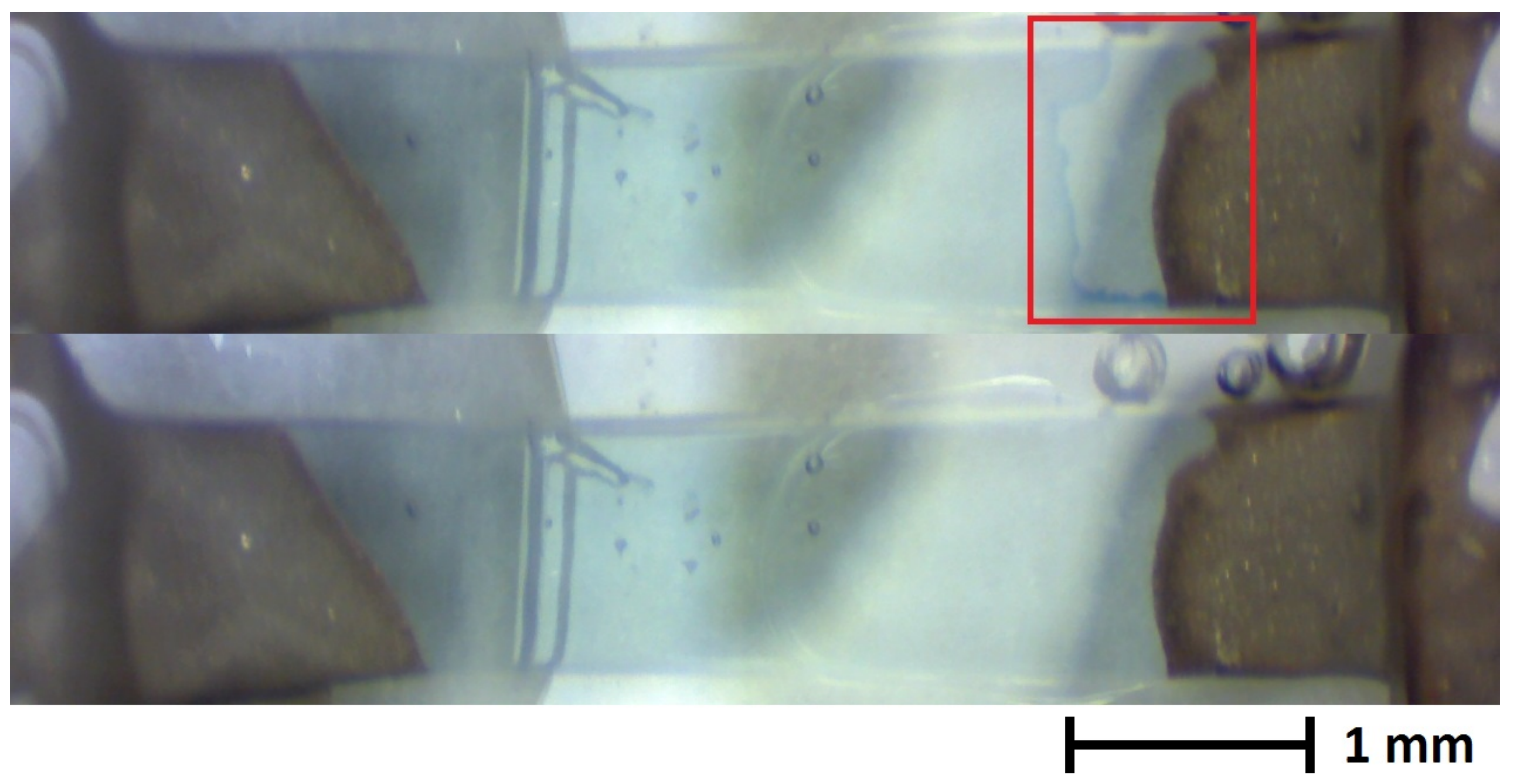

Figure 5.14: Device R1D5 with positive voltage applied to the left electrode with respect to the right electrode (top) and without (bottom).

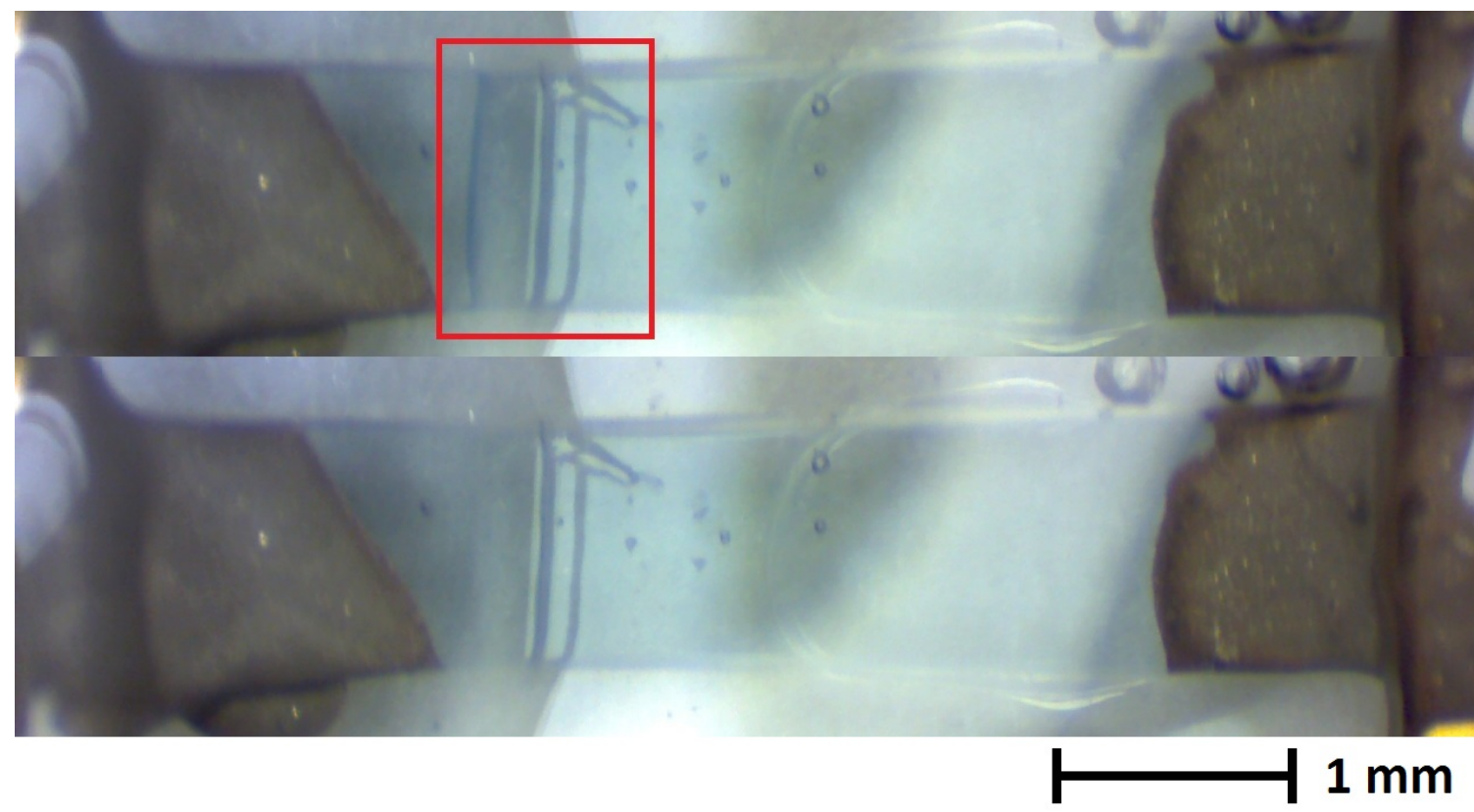

Figure 5.15: Device R1D5 with positive voltage applied to the right electrode with respect to the left electrode (top) and without (bottom). 


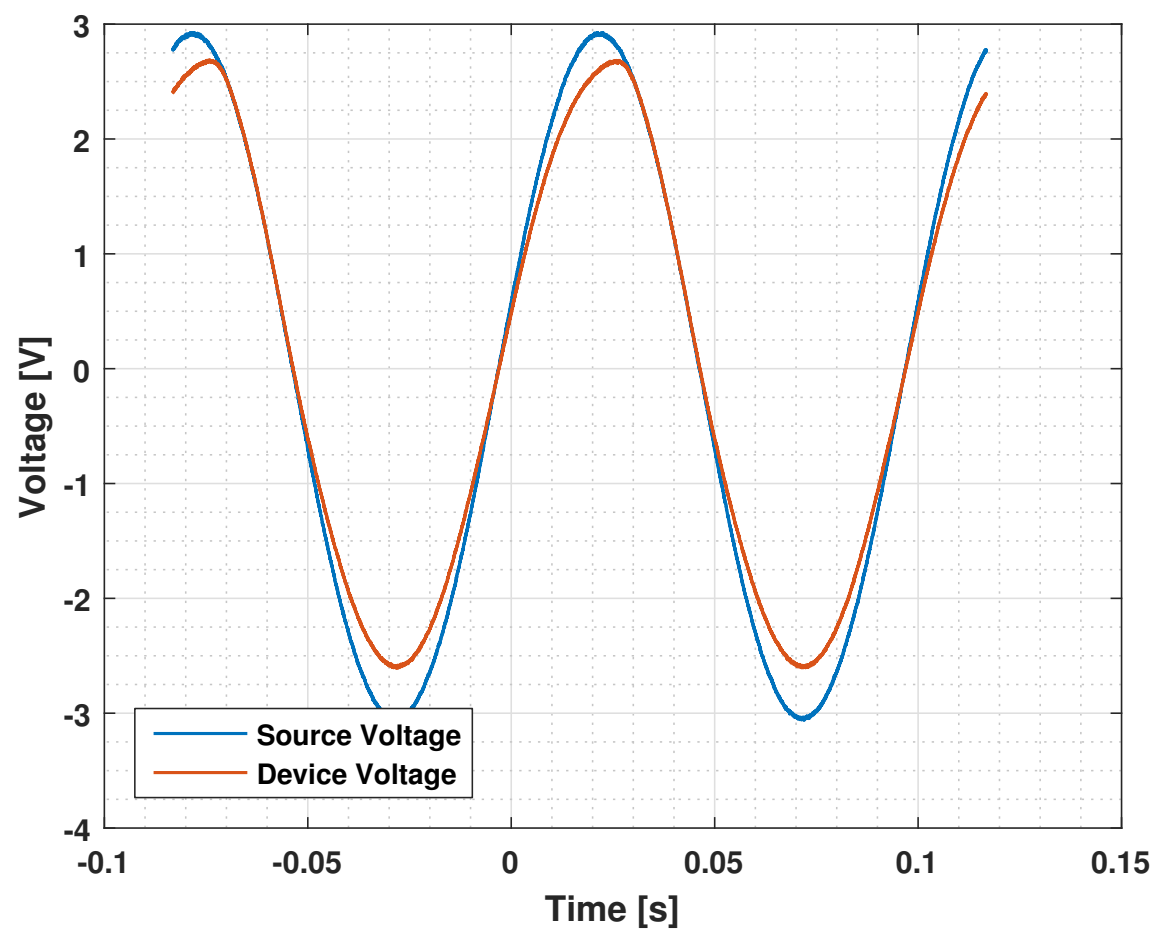

Figure 5.16: Voltage over time for testing of device R1D5 at $10 \mathrm{~Hz}, 3 \mathrm{~V}$ amplitude.

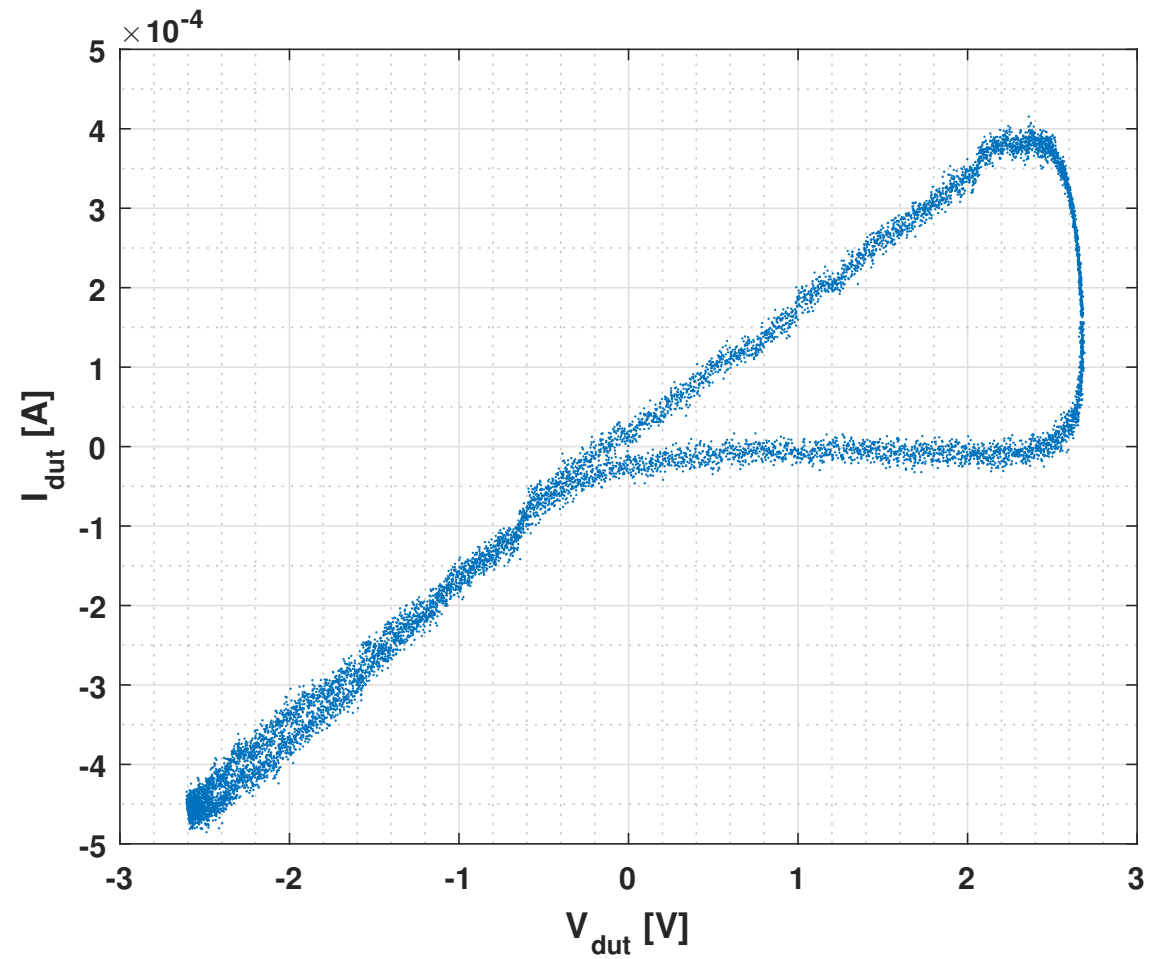

Figure 5.17: I-V curve for device R1D5 at $10 \mathrm{~Hz}$. 


\section{Device R2D2}

Device R2D2 is the first device in the second rail of devices that were intentionally fabricated with increasing widths so as to investigate the effects of wider device channels. Figures 5.18 and 5.19 summarize the fabrication of the device. The electrodes are well isolated, and the ion front is uniform so the device should operate in the expected manner.

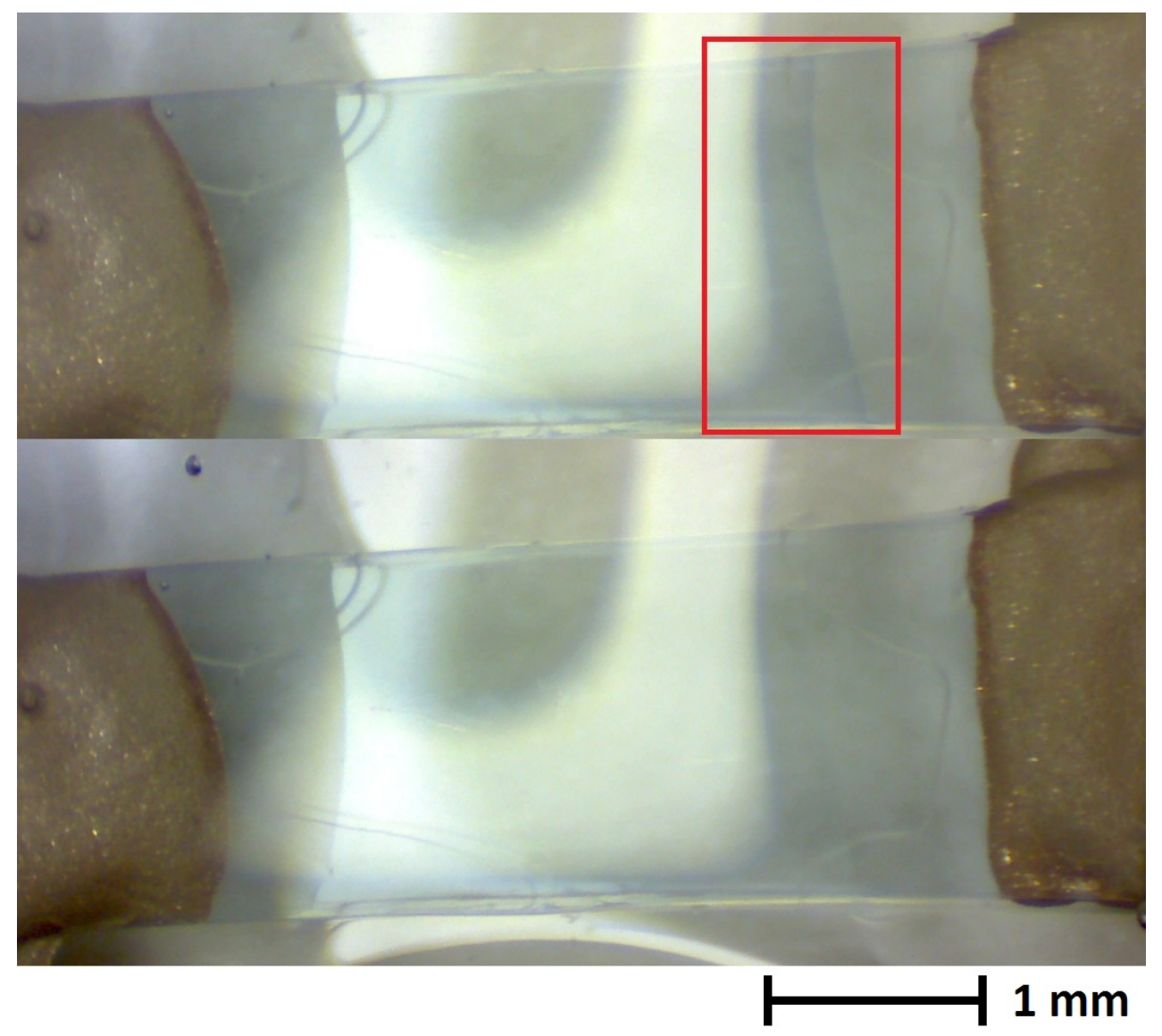

Figure 5.18: Device R2D2 with positive voltage applied to the left electrode with respect to the right electrode (top) and without (bottom). 


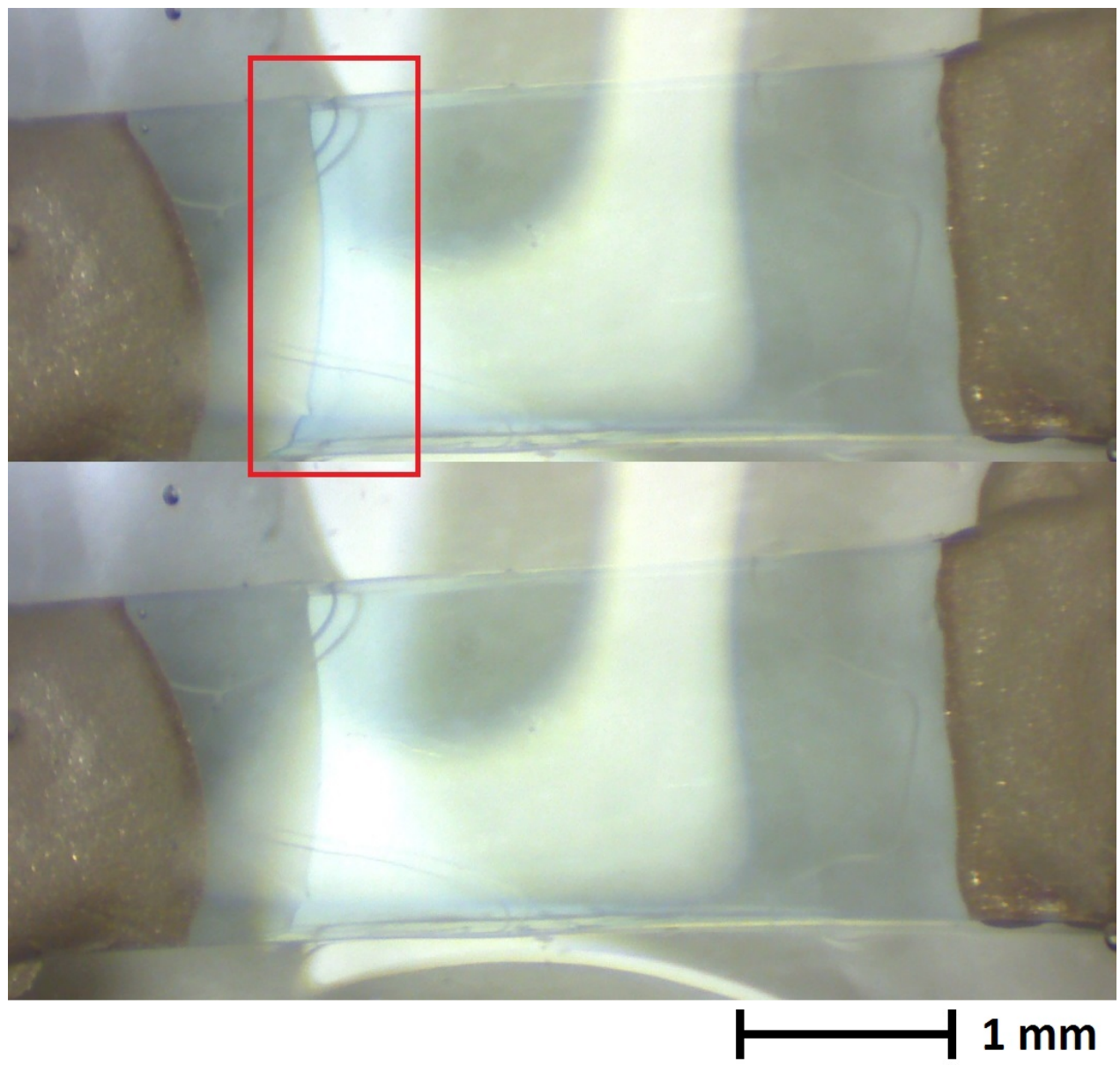

Figure 5.19: Device R2D2 with positive voltage applied to the right electrode with respect to the left electrode (top) and without (bottom).

Looking at Figures 5.20 and 5.21, however, it is obvious that the device is not operating ideally. The behaviour of the device is similar to that of device R1D5, in that it appears to operate normally on one half of the waveform but is nearly a regular resistor for the other half. Unlike R1D5, there is no readily apparent reason for this behaviour. It is possible that the device was permanently changed due to high voltages from previous tests. Devices on $\mathrm{R} 2$ were connected into the test circuit in the opposite configuration to the devices on R1. This means that the lack of response in the negative cycle is most likely a result of some problem with the left side of the device. Regardless, this one half of the device is enough to draw conclusions from 
since the devices are symmetrical.

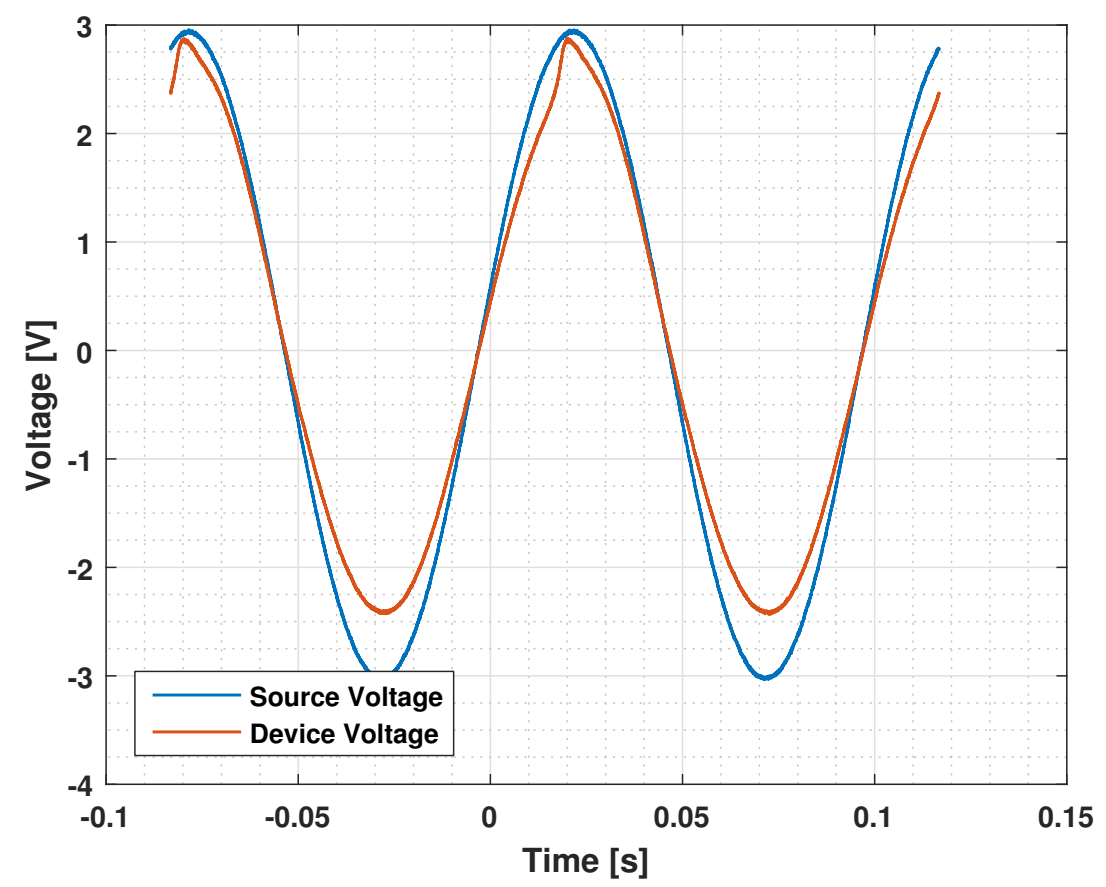

Figure 5.20: Voltage over time for testing of device R2D2 at $10 \mathrm{~Hz}, 3 \mathrm{~V}$ amplitude.

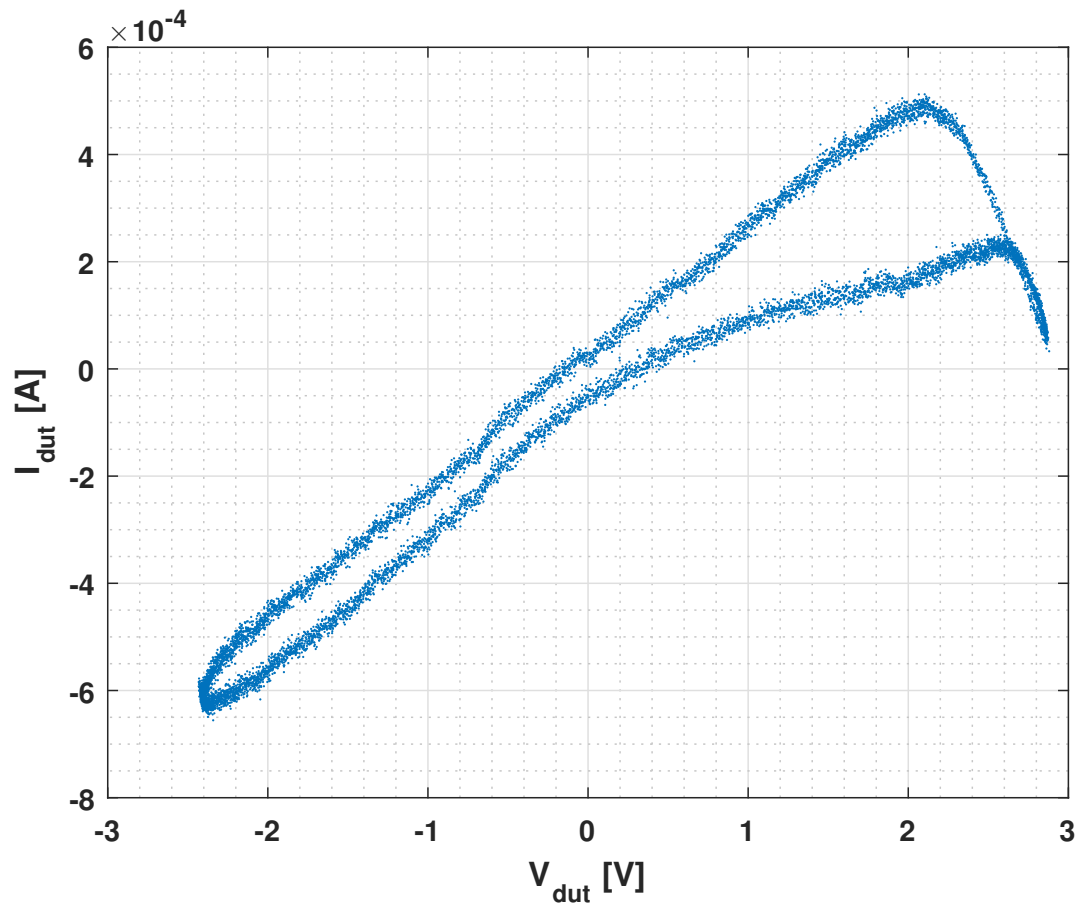

Figure 5.21: I-V curve for device R2D2 at $10 \mathrm{~Hz}$. 


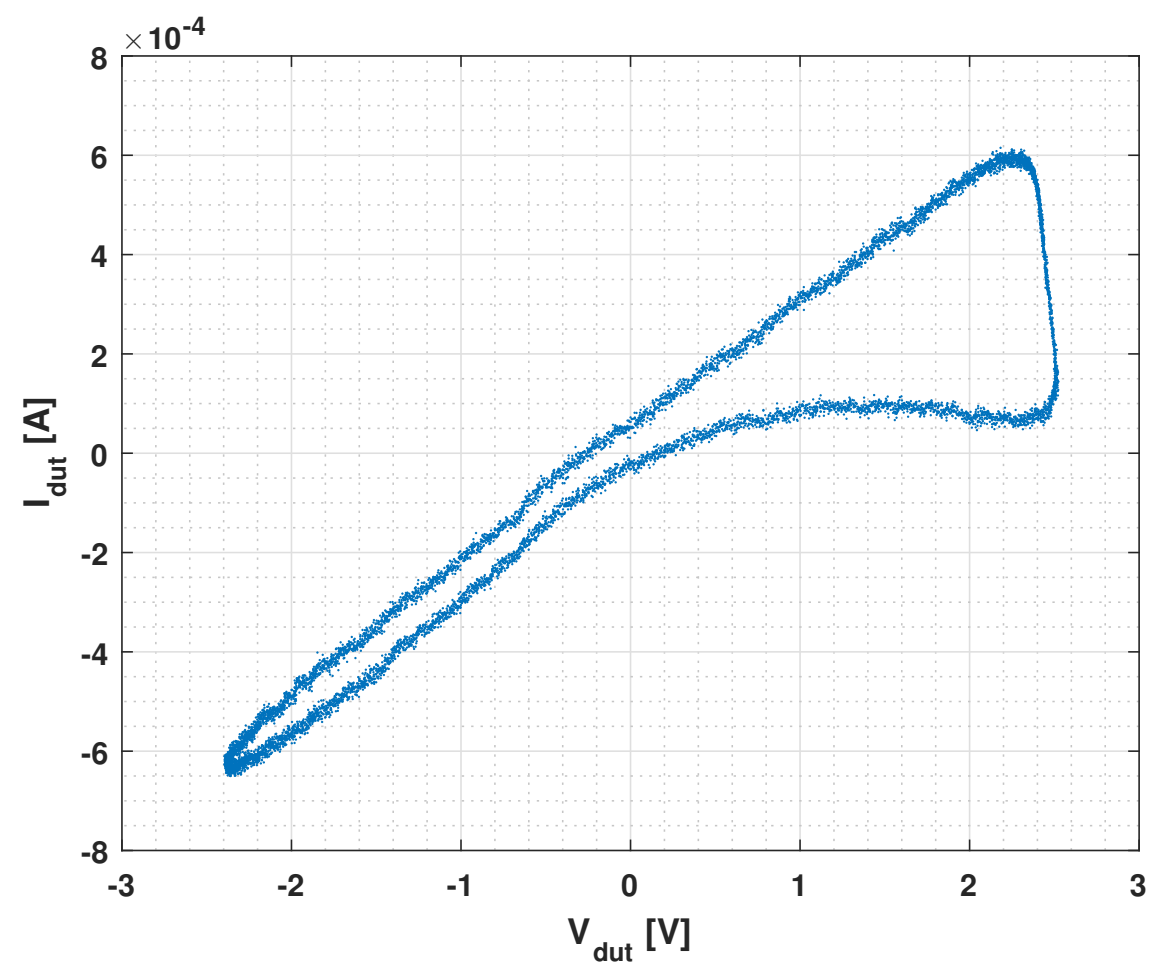

Figure 5.22: I-V curve for device R2D2 at $20 \mathrm{~Hz}$.

The width of the device seems to affect the shape of the hysteresis loop and the degree of non-linearity. Figure 5.22 shows a far more similar hysteresis response to that of device R1D5 for device R2D2 running at $20 \mathrm{~Hz}$.

\section{Device R2D3}

Device R2D3 was the result of several mistakes in fabrication being accounted for. The splatter from the application of the silver ink was accommodated by expanding the region insulated by epoxy, as shown in Figures 5.23 and 5.24. This resulted in a device with a particularly short active channel. Due to the inconsistent deposition of epoxy, and the wide device channel, the active area is wider than it is long. 


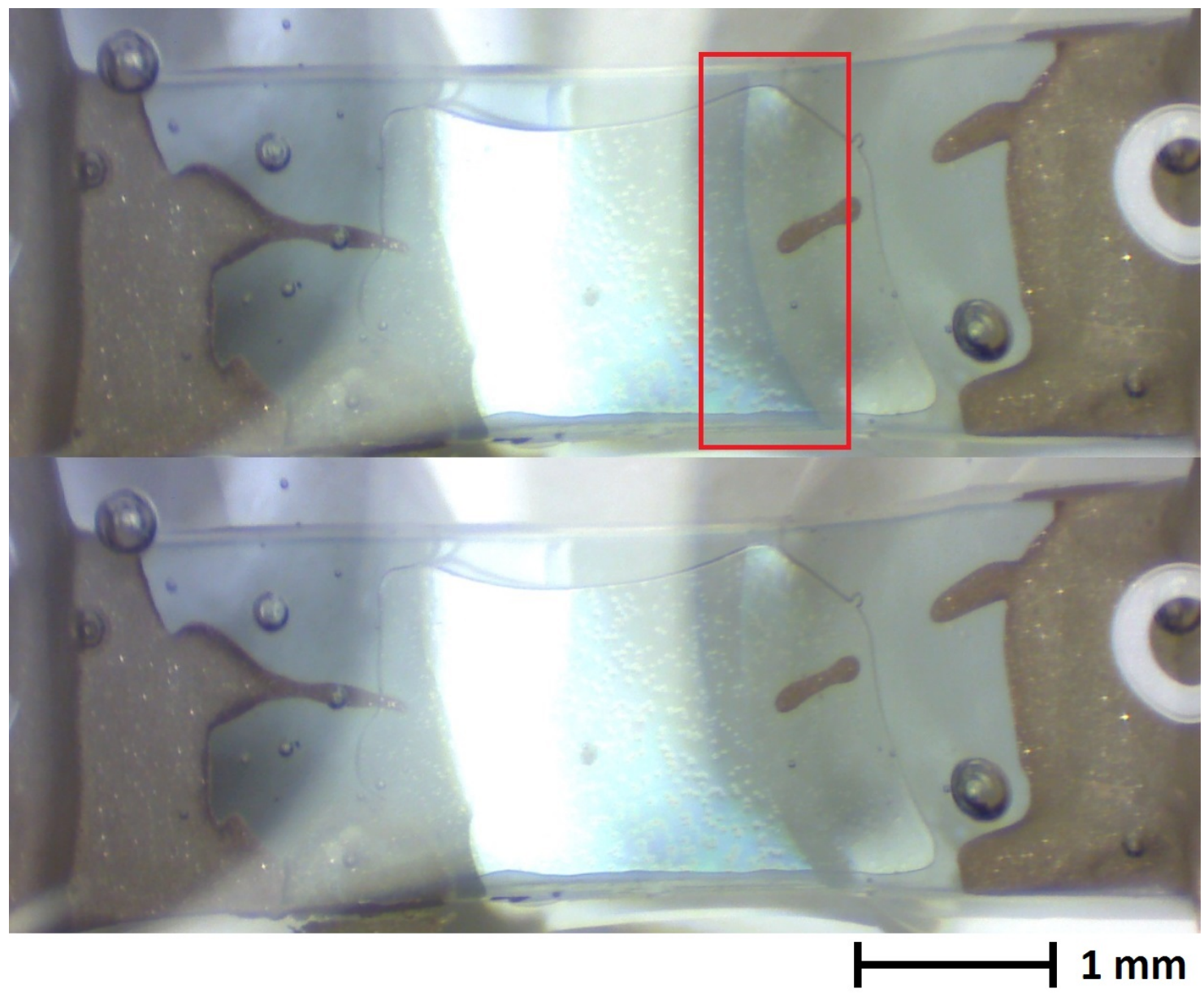

Figure 5.23: Device R2D3 with positive voltage applied to the left electrode with respect to the right electrode(top) and without (bottom). 


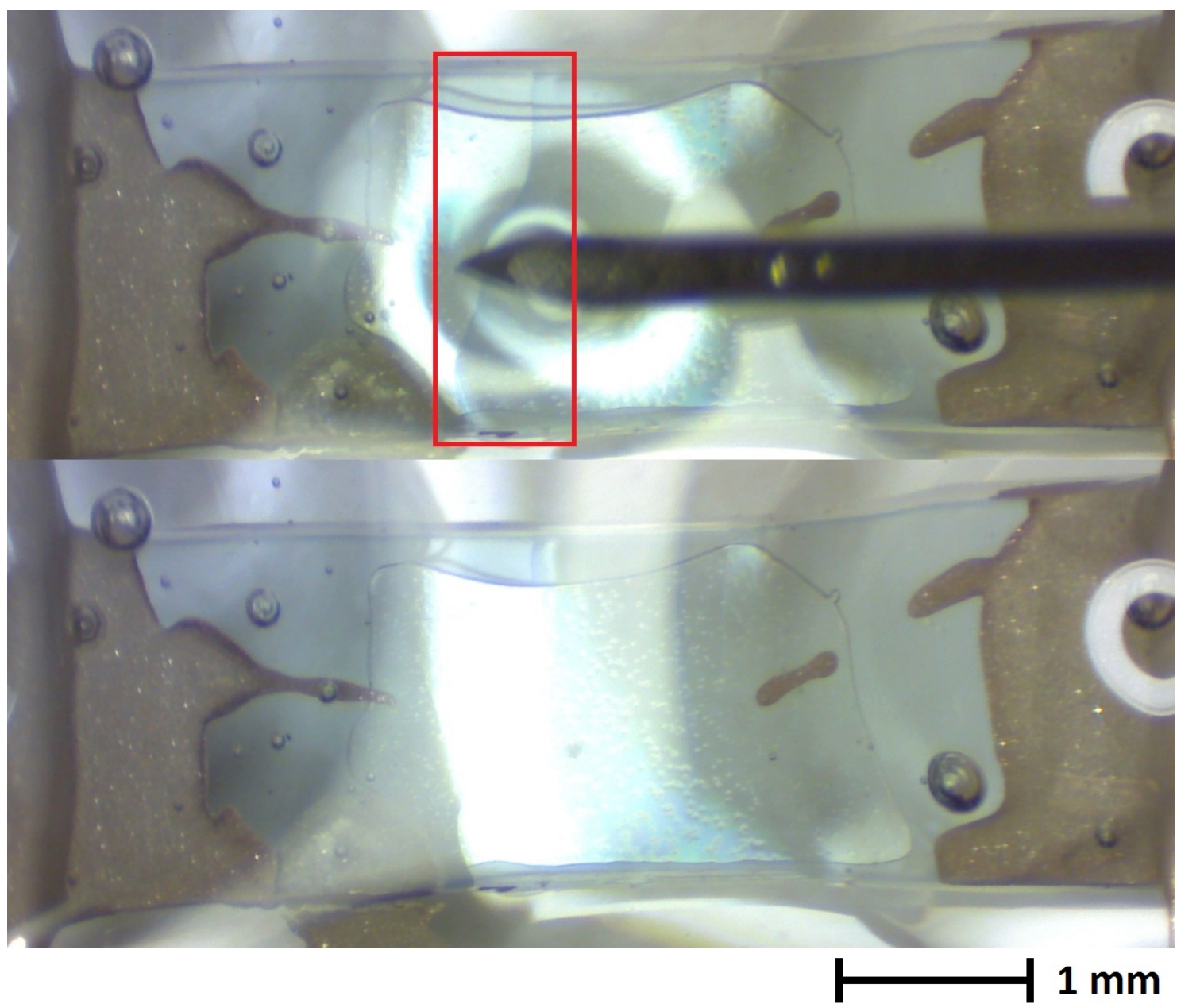

Figure 5.24: Device R2D3 with positive voltage applied to the right electrode with respect to the left electrode (top) and without (bottom). In the top half of the photo, a needle was used to distort the top of the electrolyte and reduce the shine so that the ion front could be seen.

This resulted in a hysteresis loop that did not come close to closing on itself or crossing zero. In addition, since the $L i^{+}$ions did not need to move around very much in the material, the device is not exceptionally non-linear. Though it does still display expected characteristics, they are less pronounced, as seen in Figures 5.25 and 5.26. 


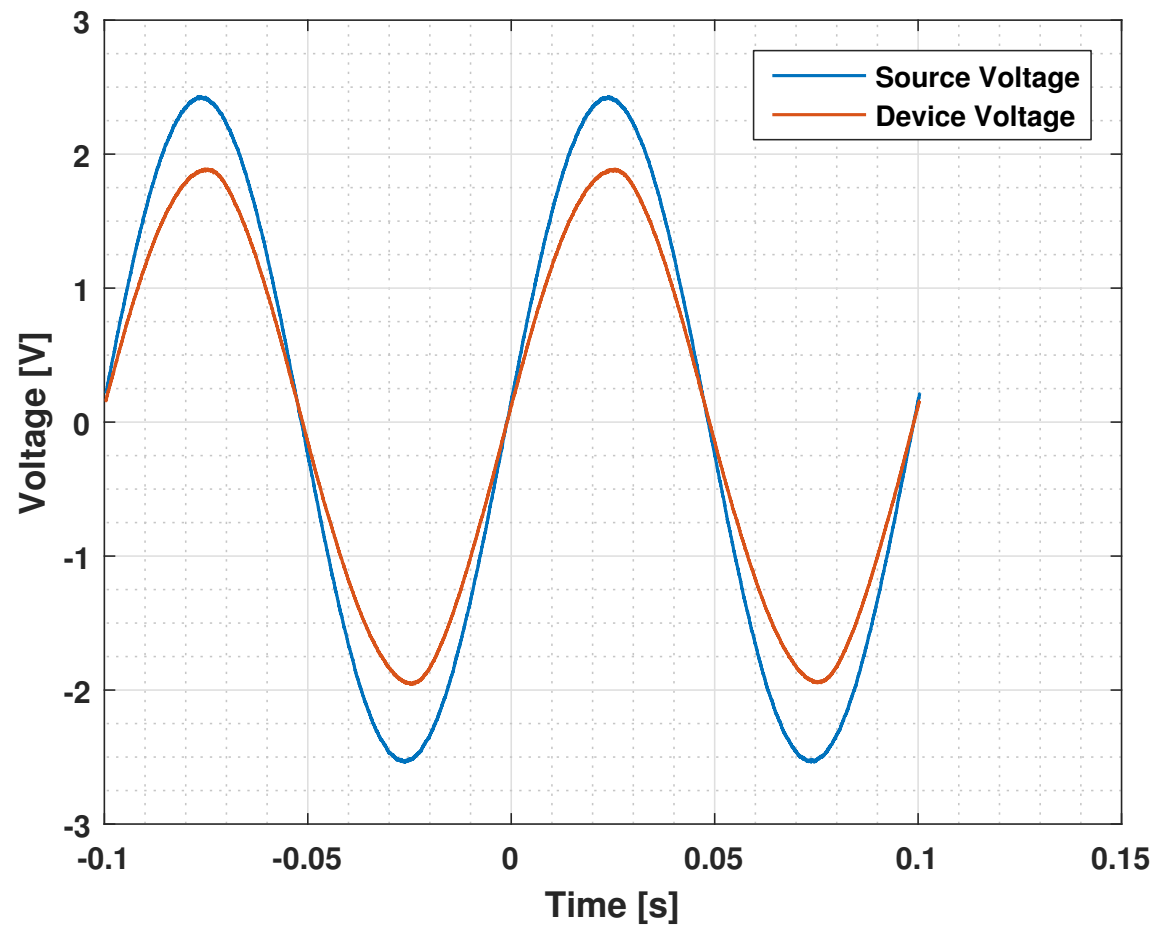

Figure 5.25: Voltage over time for testing of device R2D3 at $10 \mathrm{~Hz}, 2.5 \mathrm{~V}$ amplitude.

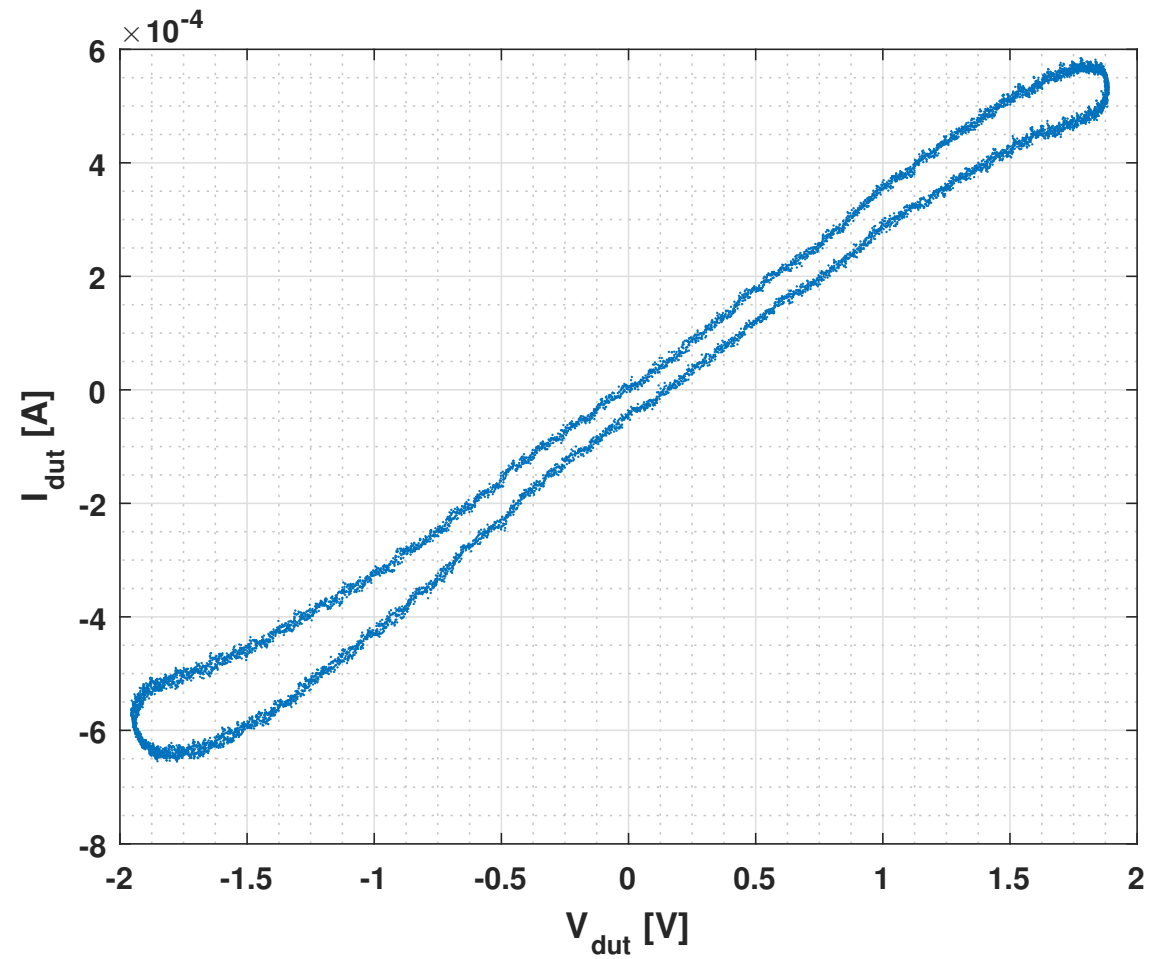

Figure 5.26: I-V curve for device R2D3 at $10 \mathrm{~Hz}$. 


\section{Device R2D4}

Device R2D4 is the widest device that was able to be fabricated by hand. Beyond this width, there was not sufficient space between the active layer and the pin header to create a proper epoxy well. The device otherwise has standard device properties, as can be seen in Figures 5.27 and 5.28.

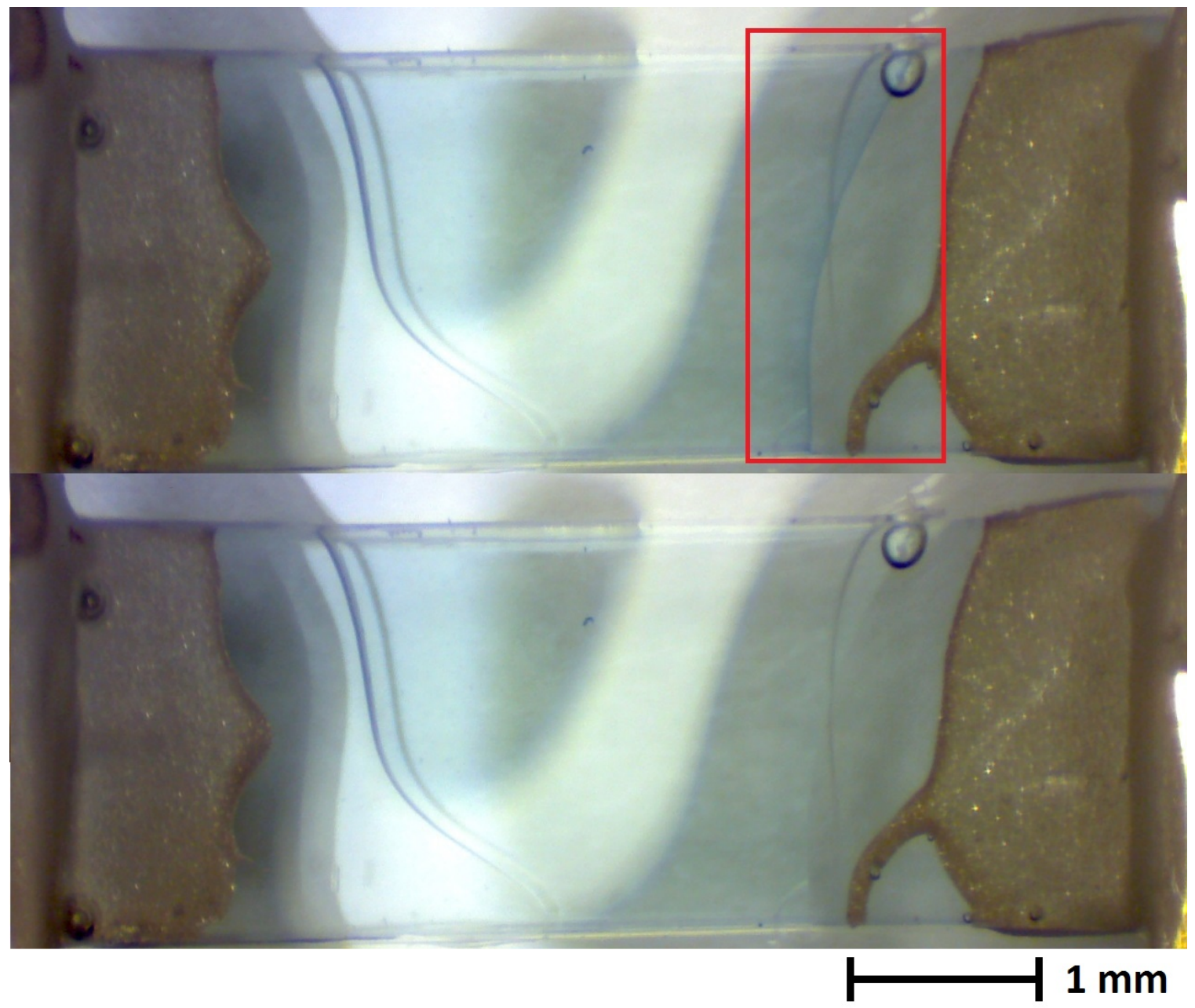

Figure 5.27: Device R2D4 with positive voltage applied to the left electrode with respect to the right electrode (top) and without (bottom). 


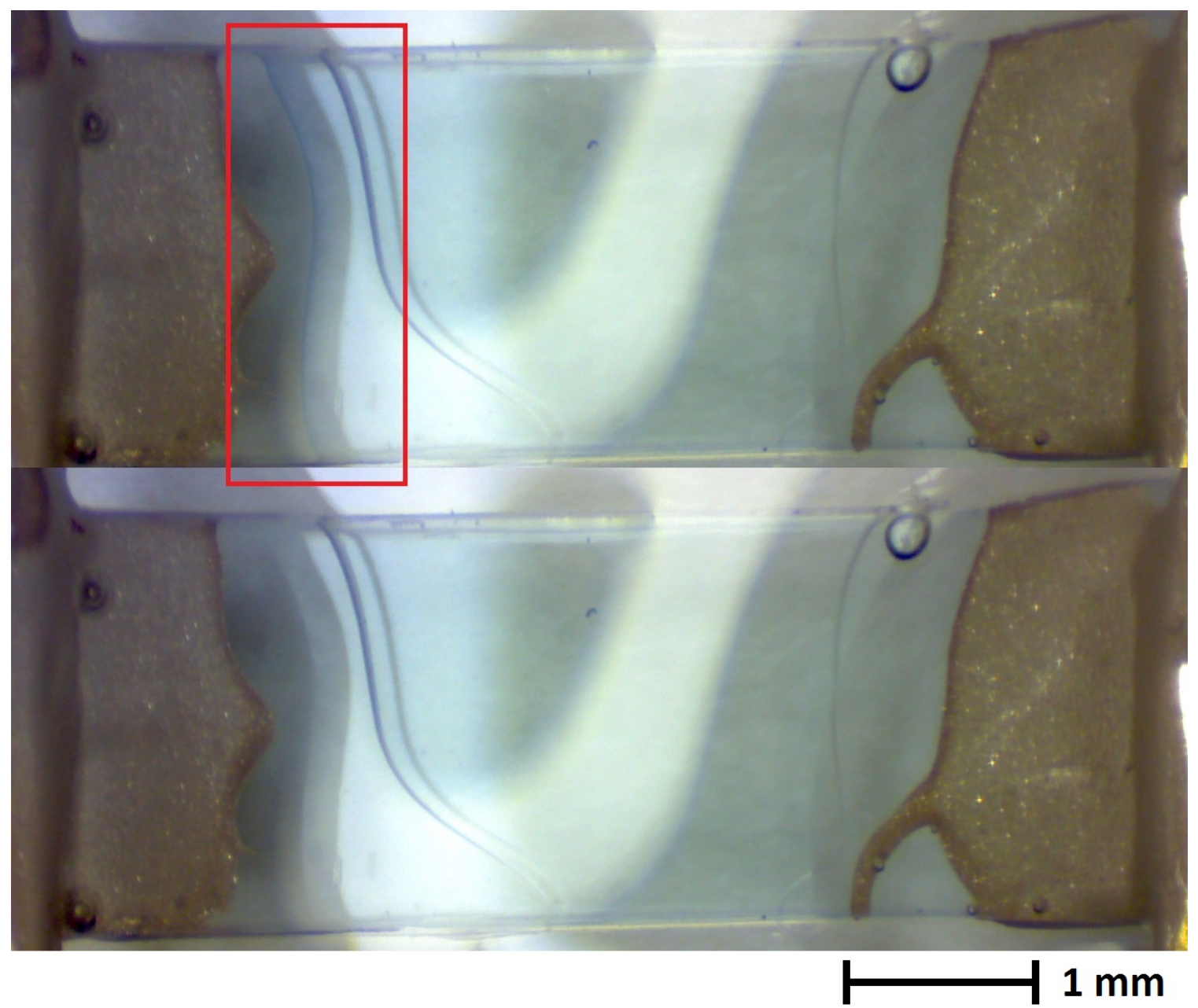

Figure 5.28: Device R2D4 with positive voltage applied to the right electrode with respect to the left electrode (top) and without (bottom).

Again, this device seems to exhibit the previously established characteristic where it is functional for one half-cycle but not the other as shown in Figures 5.29 and 5.30. As before, this is probably due to a combination of factors. The lack of non-linear response on the positive cycle seems to indicate some flaw with the right side of the device. This could be due to the proximity of the device active region to the electrode on the right side. 


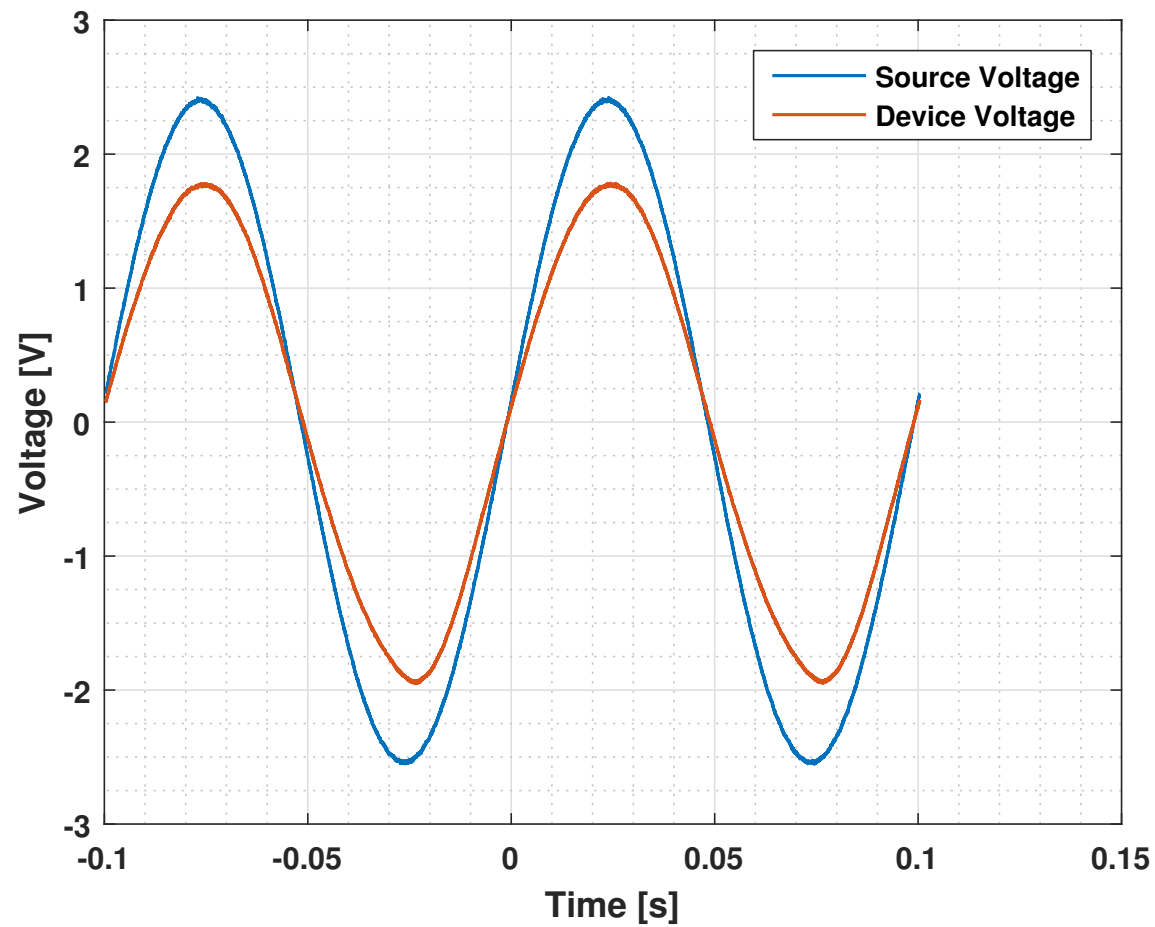

Figure 5.29: Voltage over time for testing of device R2D4 at $10 \mathrm{~Hz}, 2.5 \mathrm{~V}$ amplitude.

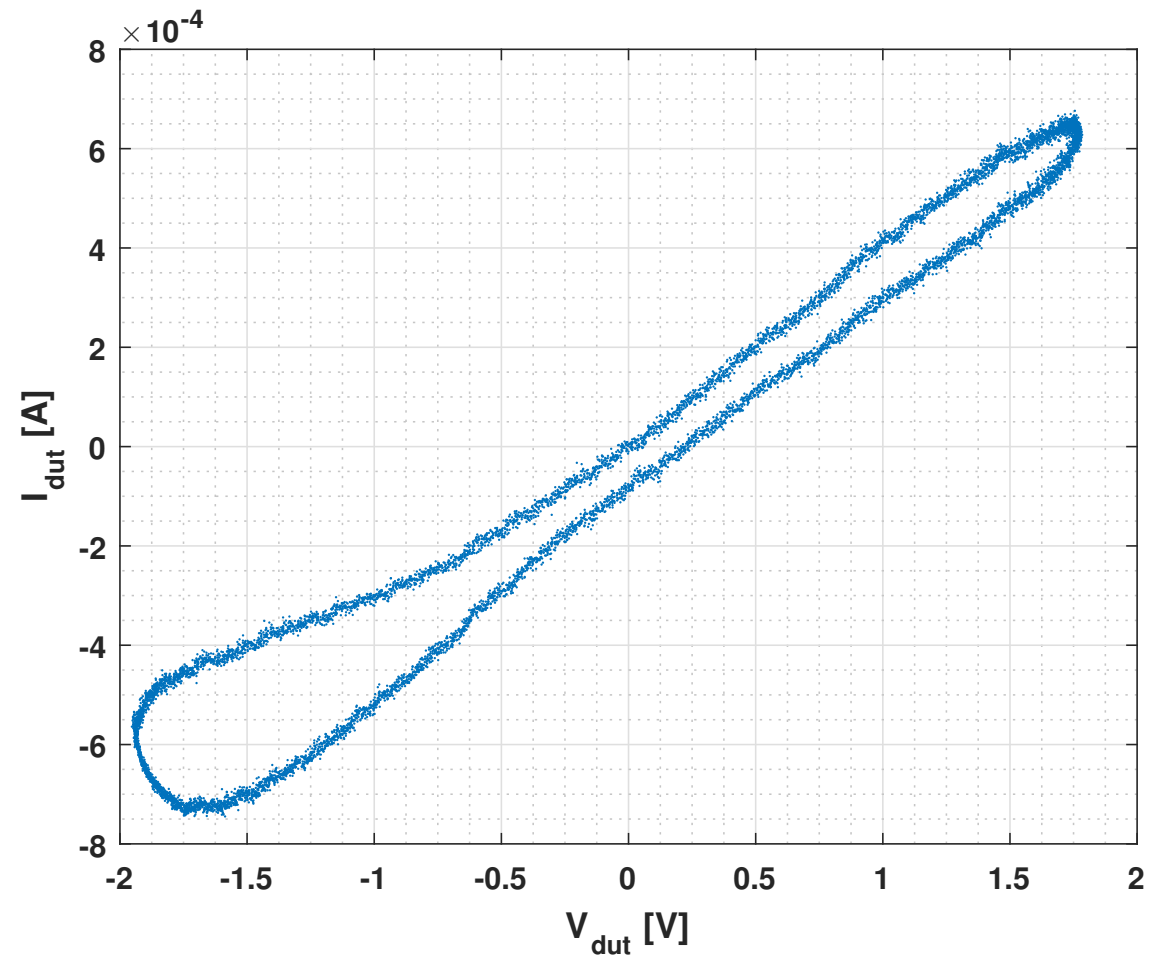

Figure 5.30: I-V curve for device R2D4 at $10 \mathrm{~Hz}$. 


\subsection{Simulation and Measurement Comparison}

This section will compare the measured response of devices to the simulation model implemented earlier in this thesis. It is reasonable to assume that these simulations will be reasonably accurate, as they were shown to match previously measured data quite well in Chapter 3. Since the majority of the testing of these devices was performed in regimes that avoided saturation, the part of the model that is most prone to error should not affect the results.

The schematic test bench that was used for the proceeding simulations is given in Figure 5.31. The only notable difference between this schematic and Figure 3.8, used previously, is accounting for the sensing resistor that would need to be used to measure the fabricated devices.

The first comparison to make is the behaviour of the devices given various frequencies of input signals. Figures 3.9 and 3.16 show composite plots of simulations of the devices with a wide range of frequencies in the ideal simulation environment. Figure 5.32 shows a similar range of frequencies simulated, but passed through a $986 \Omega$ sensing resistor, as was the scenario in measurement. It can be seen that the devices in the simulation become far more linear and display much less hysteresis than devices in the idea simulation. Comparatively, Figure 5.33 shows measured data for device R2D2 with a range of frequency inputs. Device R2D2 was selected for comparison, as the part which functioned as expected worked quite well, and other, more ideal devices had ceased functioning. It was discovered that any voltage above approximately $2 \mathrm{~V}$ will permanently degrade the device if applied for more than a few seconds.

Figure 5.33 actually shows considerably more non-linearity and hysteresis than the simulated data across a range of frequencies. This is most likely due to the fact that the fabricated devices had a higher average resistance value than those in simulation, 


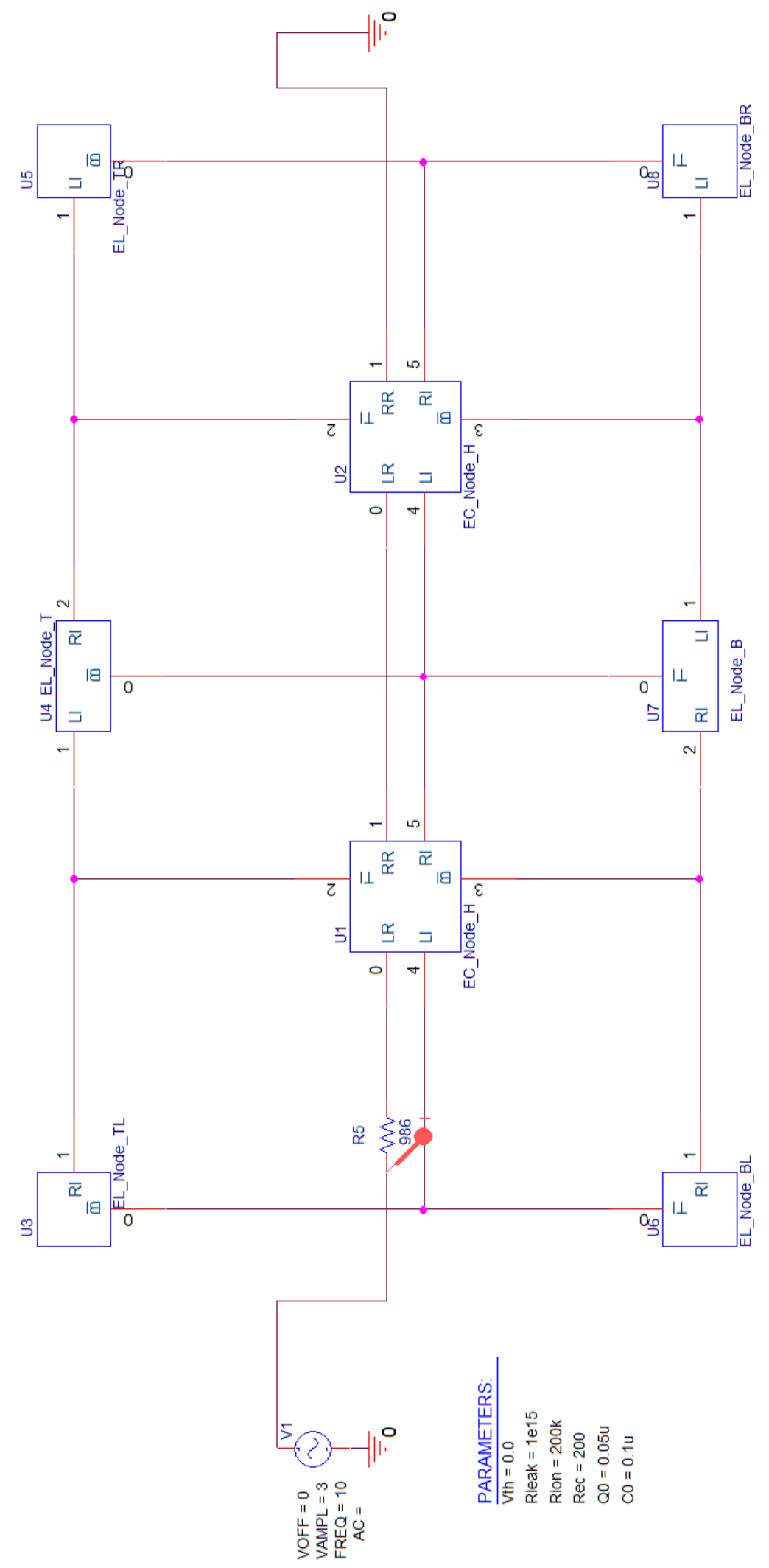

Figure 5.31: Simulation circuit schematic used for comparison to real device tests to account for sensing resistor. 
which was around $2 \mathrm{k} \Omega$, causing a proportionately larger input voltage to drop across the sensing resistor. In addition, the data captured for low frequencies (below $10 \mathrm{~Hz}$ ) is significantly noisier than that of higher frequencies. This was due to a setting on the oscilloscope that causes the display to automatically sweep for data if the trigger condition was not found within a certain time frame. Unfortunately this problem was not realized at the time of measurement. The average behaviour of the signal remains valid. Comparing Figure 5.32 to 5.33, it is clear that their overall behavioural trends are the same. They both demonstrate a widening of the hysteresis loop around a certain frequency (approximately $10 \mathrm{~Hz}$ ), which degrades at lower frequency to more of a sigmoid shape. They also both demonstrate that as the frequency increases, the devices become functionally equivalent to linear resistors as expected. This also matches reasonably well with the previous measurements within the group, seen in Figure 3.15.

As expected, the simulations and measured data across a frequency range match reasonably well, but not perfectly, particularly at lower frequency due to the lack of accounting for device saturation and de-saturation. 


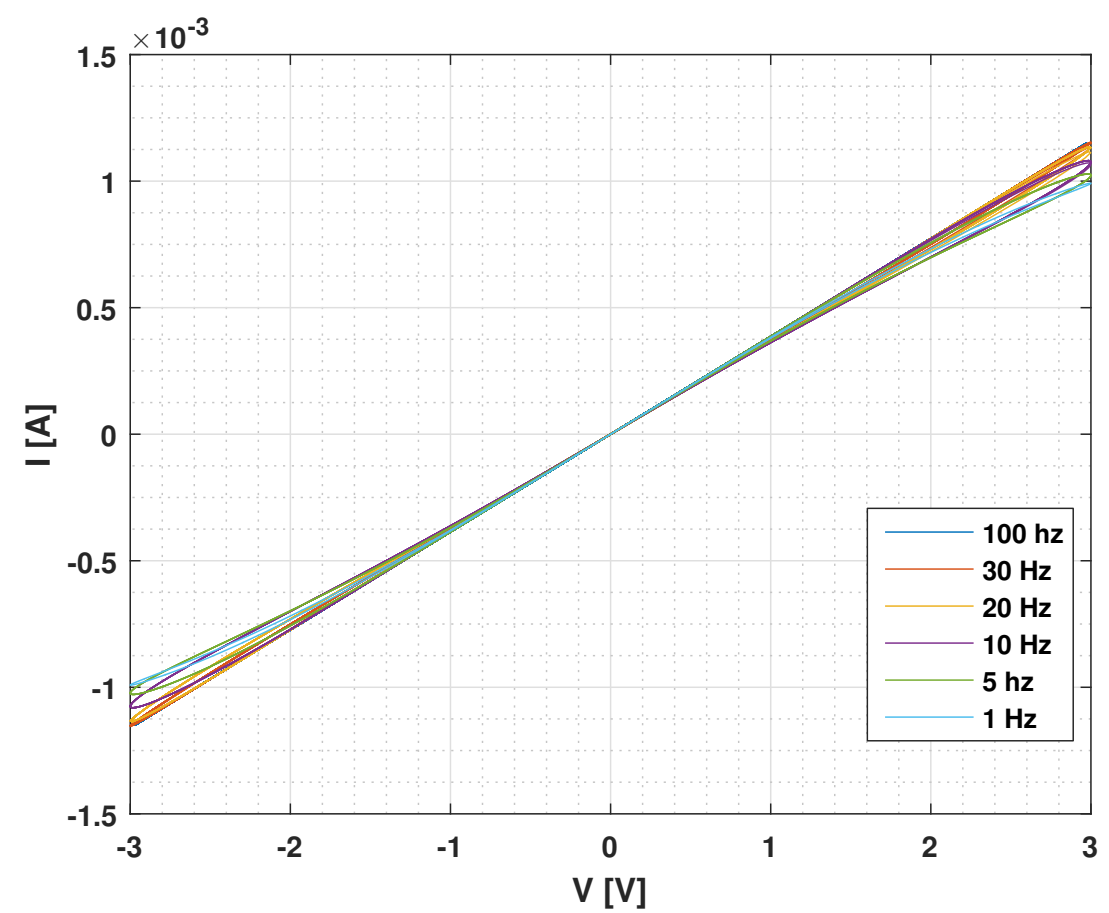

Figure 5.32: I-V characteristic curves for simulated devices with varying frequency inputs.

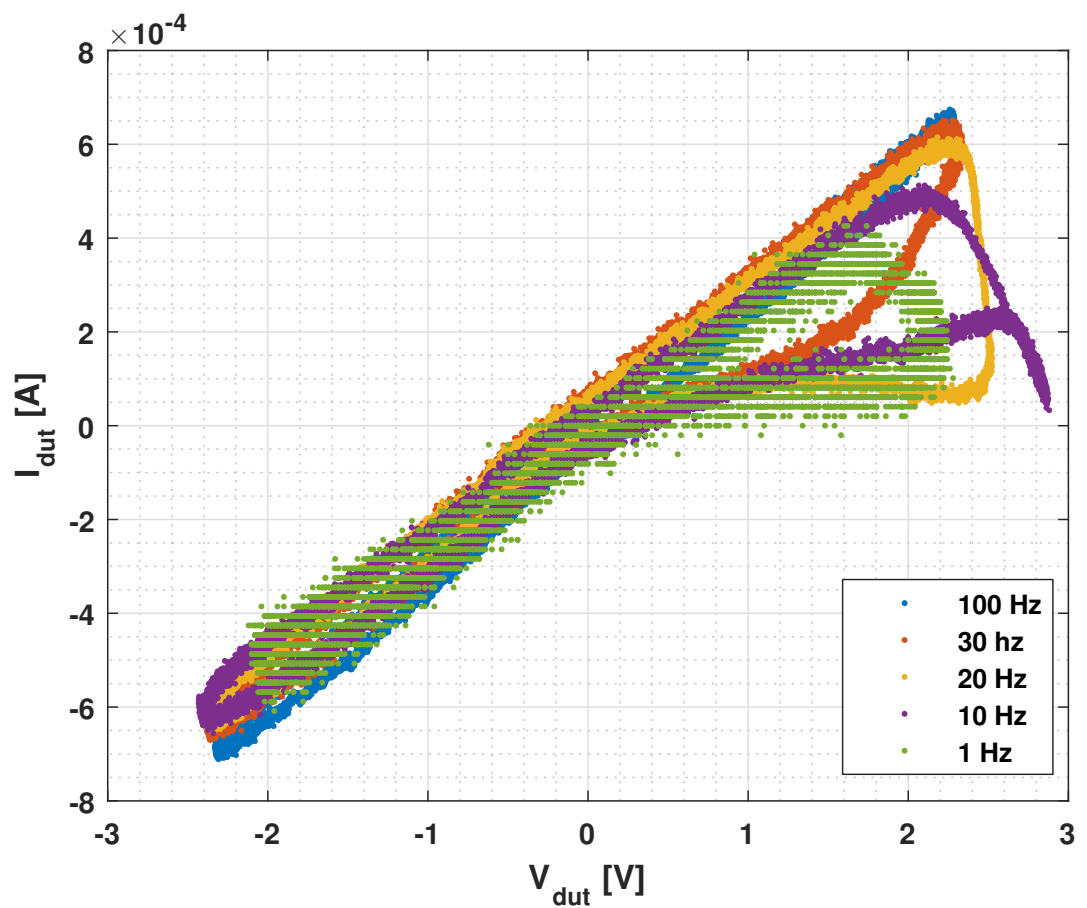

Figure 5.33: I-V characteristic curves measured on device R2D2 with varying frequency inputs. 
The next point of comparison is the behaviour of the devices at a range of input signal amplitudes at a frequency of $10 \mathrm{~Hz}$. Two devices were tested to compare this behaviour: device R1D5 and device R2D3. Figure 5.34 shows the simulated data for a device measured via a sensing resistor at several input amplitudes. Compared to Figure 5.35, a plot of the same input signal amplitudes measured over device R1D5, the behavioural match is quite strong. Device R1D5 also had hysteresis on only one half-cycle, but the comparison remains useful since generally speaking, the device would normally be symmetrical. The $4.5 \mathrm{~V}$ input amplitude I-V trace in Figure 5.35 was captured with the trigger setting unfixed, but even so, the average of the data correlates with the trend of a widening hysteresis loop that can be seen in both the simulated and measured data. There is once again some numerical mismatch due to the lack of compensation for the actual average resistance of the device as compared to the simulated device.

This match between measured and simulated data can also be seen readily for device R2D3. Figure 5.36 provides the simulated I-V traces for a device, and Figure 5.37 shows the measured data for the device across a range of input amplitudes. In this case, a properly symmetrical device was used to acquire the data. It can be seen that the data in the two figures matches incredibly well, apart from the anomaly in the shape of the waveform when maximum or minimum input voltages are reached on the measured device. This effect is likely an indicator of device breakdown. Only a few cycles could be captured at these higher voltages before the device would be destroyed. This also means that each higher voltage measurement is slightly affected by the previous ones as they would degrade the device for the few cycles they were applied. Prolonged application resulted in the device ceasing to conduct any current whatsoever. 


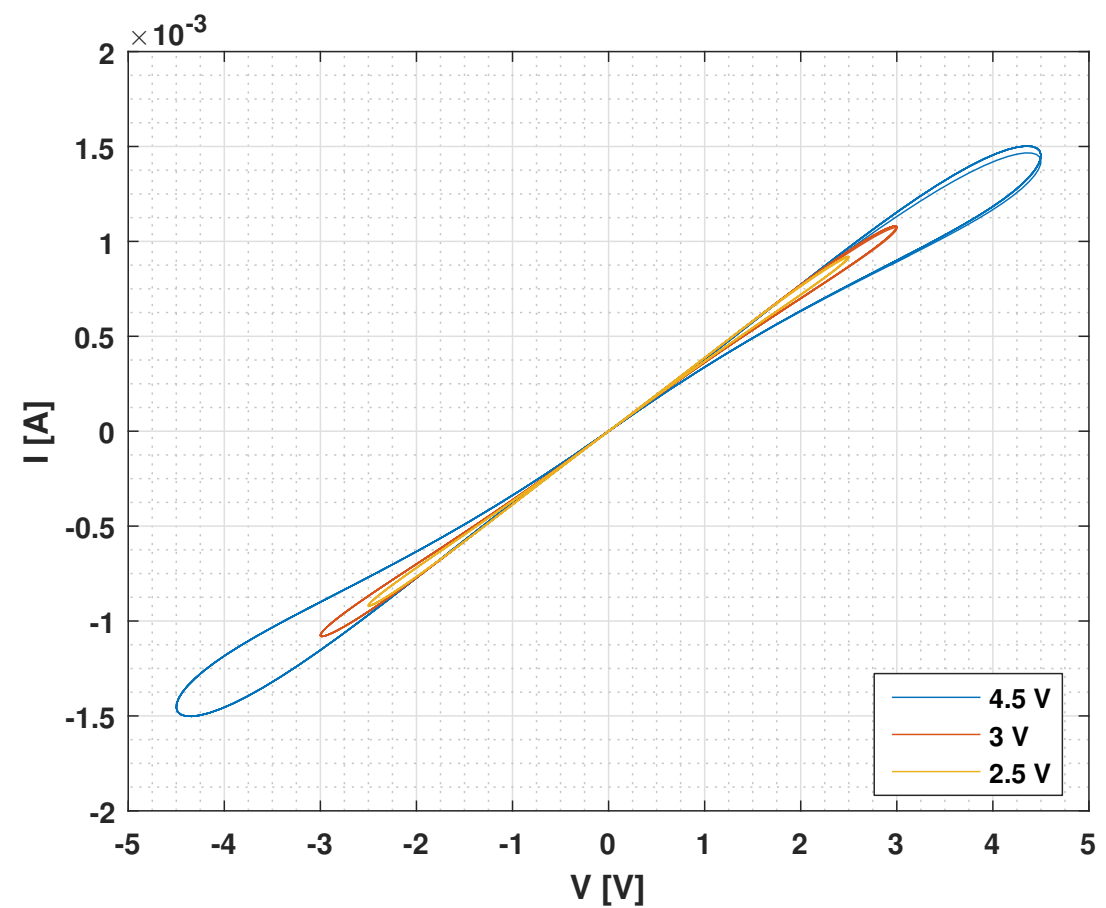

Figure 5.34: I-V characteristic curves for simulated devices with varying voltage amplitude inputs to compare with R1D5.

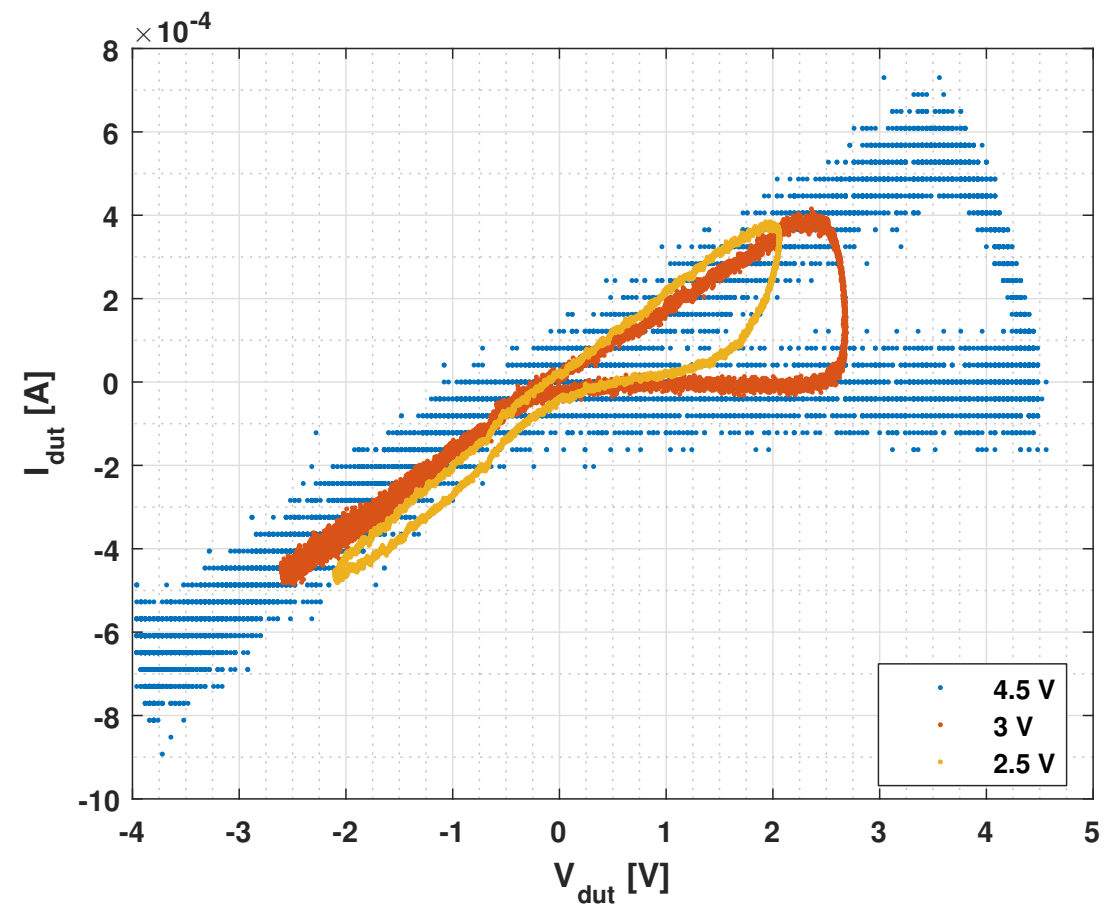

Figure 5.35: I-V characteristic curves measured on device R1D5 with varying voltage amplitude inputs. 


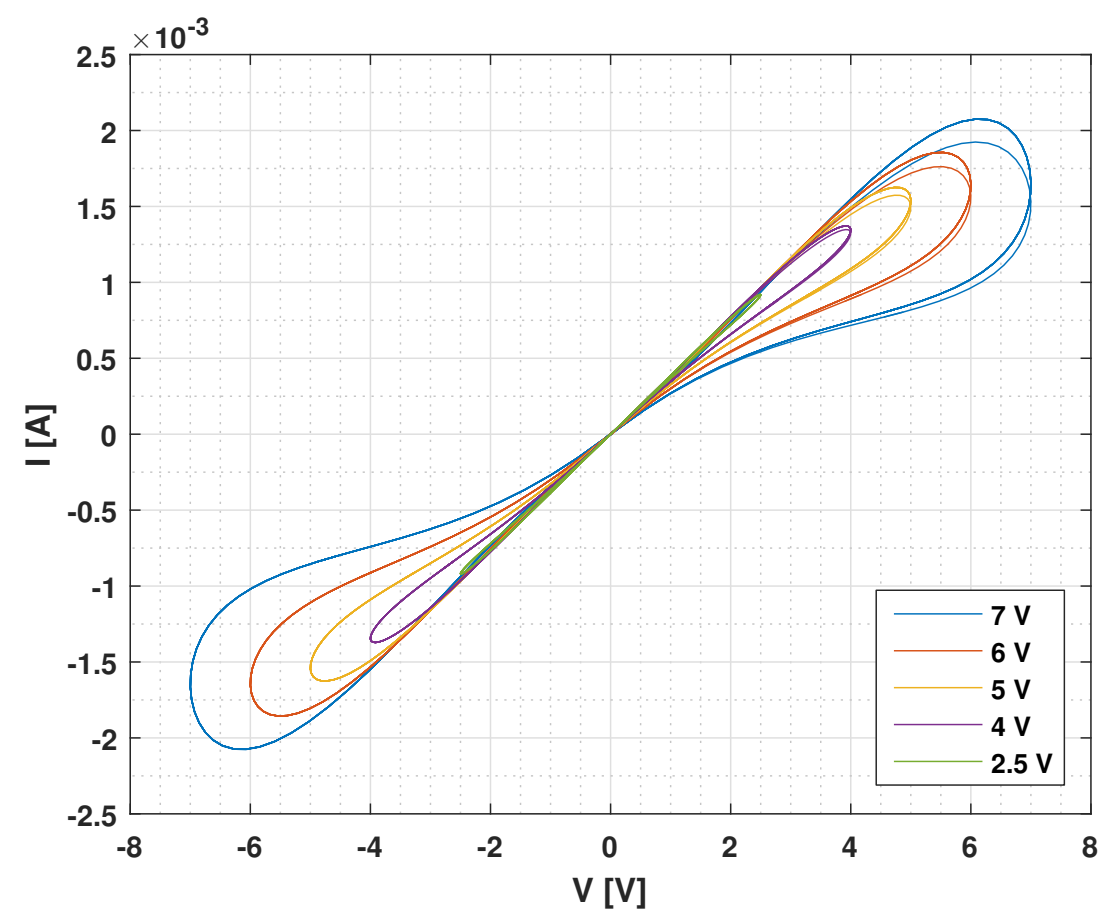

Figure 5.36: I-V characteristic curves for simulated devices with varying voltage amplitude inputs to compare with R2D3.

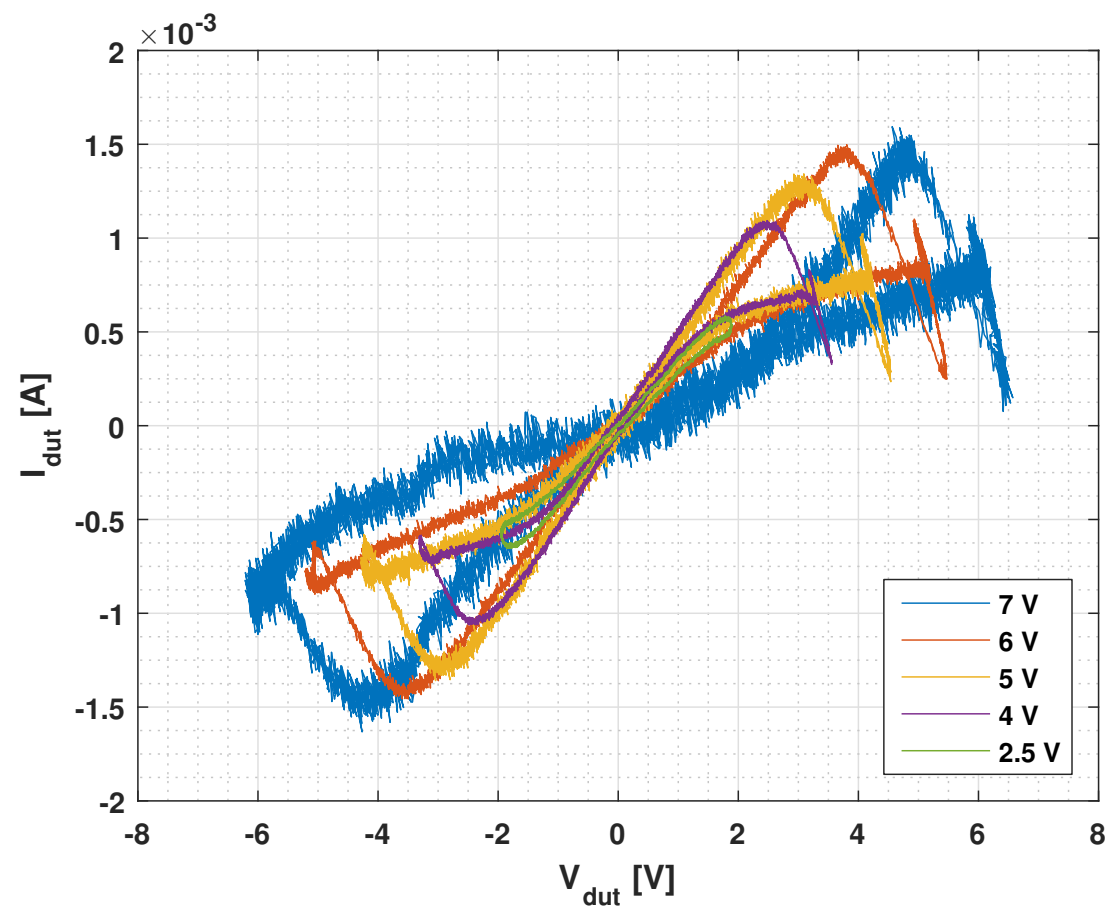

Figure 5.37: I-V characteristic curves measured on device R2D3 with varying voltage amplitude inputs. 


\section{Chapter 6}

\section{Conclusion}

\subsection{Summary}

The objective of this thesis was to create a simple and efficient method to simulate ionically-coupled, non-linear devices with reasonable accuracy that could be scaled up for use in modelling larger, more complex neuropmorphic circuits, as well as to verify the accuracy of these simulations via the fabrication and testing of these devices.

This work presented experimental results for the comparison and validation of a simplified, SPICE-based non-linear resistor model. Comparisons were drawn to validate the accuracy and range of a semi-empirical model that can be implemented using currently available simulation software and that uses significantly less computational resources than more complex models. A method of fabricating corresponding devices was also presented using commonly available and well-established materials.

These devices were tested and their functionality evaluated. The behaviour of these devices was discussed, and any abnormalities investigated. The functionality of the devices was then compared to that of the previously validated simulations, and strong correlations were found. 


\subsection{Future Work}

Looking forward, parameter extraction should be performed on the devices to more closely relate the results of the simulations with those of the devices themselves. In addition, some level of granularity in the model would aid in the accuracy of its results. In addition, the process for fabricating these devices would benefit greatly from some level of automation. Consistency of device quality and yield are difficult factors to maintain high standards on when work of this scale is being performed by hand.

These devices have the potential to see a myriad of uses in the field of neuromorphic applications and CNNs. Development of these devices into cells that could be scaled into large matrices would be ideal next steps. Even the electrochromic aspects of these devices could be used, perhaps as a means of sensing the state of the device without the need of a probing or testing voltage. Neuromorphic networks using nonlinear cells, such as the ones easily implemented by the devices studied in this thesis, are already being investigated for use in applications such as learning networks and networks that mimic the neural paths of human tissue such as the retina. 


\section{List of References}

[1] L. O. Chua and T. Roska, "The CNN paradigm," IEEE Transactions on Circuits and Systems I: Fundamental Theory and Applications, vol. 40, no. 3, pp. 147-156, Mar 1993.

[2] L. O. Chua, T. Roska, and T. Kozek, "Cellular neural networks - a tutorial on programmable nonlinear dynamics in space," in Proceedings on Analysis of Dynamical and Cognitive Systems, Advanced Course. London, UK, UK: Springer-Verlag, 1995, pp. 53-73. [Online]. Available: http://dl.acm.org/citation.cfm?id=647428.723564

[3] L. O. Chua and L. Yang, "Cellular neural networks: applications," IEEE Transactions on Circuits and Systems, vol. 35, no. 10, pp. 1273-1290, Oct 1988.

[4] K. Karahaliloglu, S. Balkir, S. Pramanik, and S. Bandyopadhyay, "A quantum dot image processor," IEEE Transactions on Electron Devices, vol. 50, no. 7, pp. 1610-1616, July 2003.

[5] C. Mead, "Neuromorphic electronic systems," Proceedings of the IEEE, vol. 78, no. 10, pp. 1629-1636, Oct 1990.

[6] A. Zarandy and C. Rekeczky, "Bi-i: a standalone ultra high speed cellular vision system," IEEE Circuits and Systems Magazine, vol. 5, no. 2, pp. 36-45, 2005.

[7] T. Roska, "Cellular wave computers for brain-like spatial-temporal sensory computing," IEEE Circuits and Systems Magazine, vol. 5, no. 2, pp. 5-19, 2005.

[8] L. Chua, "Memristor-the missing circuit element," Circuit Theory, IEEE Transactions on, vol. 18, no. 5, pp. 507-519, Sep 1971.

[9] J. M. Tour and T. He, "Electronics: The fourth element," Nature, vol. 453, pp. 42-43, May 2008. [Online]. Available: http://dx.doi.org/10.1038/453042a 
[10] L. O. Chua and S. M. Kang, "Memristive devices and systems," Proceedings of the IEEE, vol. 64, no. 2, pp. 209-223, Feb 1976.

[11] Y. Pershin and M. Di Ventura, "Memory effects in complex materials and nanoscale systems," Advances in Physics, vol. 60, no. 2, pp. 145-227, 2011.

[12] D. B. Strukov, G. S. Snider, D. R. Stewart, and R. S. Williams, "The missing memristor found," Nature, vol. 453, pp. 80-83, May 2008. [Online]. Available: http://dx.doi.org/10.1038/nature06932

[13] S. Vongehr and X. Meng, "The missing memristor has not been found," Scientific Reports, vol. 5, June 2015.

[14] L. Chua, "Resistance switching memories are memristors," Applied Physics A, vol. 102, no. 4, pp. 765-783, 2011. [Online]. Available: http: //dx.doi.org/10.1007/s00339-011-6264-9

[15] S. Kirchenmeyer and K. Reuter, "Scientific importance, properties and growing applications of poly(3,4-ethylenedioxythiophene)," Journal of Materals Chemistry, vol. 15, pp. 2077-2088, 2005.

[16] M. Chason, P. W. Brazis, J. Zhang, K. Kalyanasundaram, and D. R. Gamota, "Printed organic semiconducting devices," Proceedings of the IEEE, vol. 93, no. 7, pp. 1348-1356, July 2005.

[17] L. Groenendaal, F. Jonas, D. Freitag, H. Pielartzik, and J. R. Reynolds, "Poly(3,4-ethylenedioxythiophene) and its derivatives: Past, present, and future," Advanced Materials, vol. 12, no. 7, pp. 481-494, 2000. [Online]. Available: http://dx.doi.org/10.1002/(SICI)1521-4095(200004)12:7〈481:: AID-ADMA481>3.0.CO;2-C

[18] S. P. McGarry and N. G. Tarr, "Fabrication and modelling of screenprinted active electrolytic polymer devices," Semiconductor Science and Technology, vol. 23, no. 5, p. 055009, 2008. [Online]. Available: http: //stacks.iop.org/0268-1242/23/i=5/a=055009

[19] S. Timpanaro, M. Kemerink, F. Touwslager, M. D. Kok, and S. Schrader, "Morphology and conductivity of pedot/pss films studied by scanningtunneling microscopy," Chemical Physics Letters, vol. 394, no. 46, pp. 339 - 343, 2004. [Online]. Available: http://www.sciencedirect.com/science/article/pii/ S000926140401070X 
[20] T. Stcker, A. Khler, and R. Moos, "Why does the electrical conductivity in pedot:pss decrease with pss content? a study combining thermoelectric measurements with impedance spectroscopy," Journal of Polymer Science Part B: Polymer Physics, vol. 50, no. 14, pp. 976-983, 2012. [Online]. Available: http://dx.doi.org/10.1002/polb.23089

[21] A. Elschner, S. Kirchenmeyer, W. Lövenich, and K. Reuter, PEDOT: principles and applications of an intrinsically conductive polymer. Boca Raton, FL: CRC Press, 2011.

[22] A. M. Nardes, M. Kemerink, R. A. Janssen, J. A. Bastiaansen, N. M. Kiggen, B. M. Langeveld, A. J. van Breemen, and M. M. de Kok, "Microscopic understanding of the anisotropic conductivity of pedot: Pss thin films," Advanced Materials, vol. 19, no. 9, pp. 1196-1200, 2007.

[23] A. J. Heeger, N. S. Sariciftci, and E. B. Namdas, Semiconducting and metallic polymers. Oxford: Odford University Press, 2010.

[24] W. R. McKinnon and R. R. Haering, Modern Aspects of Electrochemistry: No. 15. Boston, MA: Springer US, 1983, ch. Physical Mechanisms of Intercalation, pp. 235-304. [Online]. Available: http://dx.doi.org/10.1007/978-1-4615-7461-3_4

[25] E. B. Ramirez, "Characterization of a PEDOT:PSS electrolytic device using an in situ spectroelectrochemical technique," M. A. Sc., Carleton University, Ottawa, Ontario, Canada, 2013.

[26] C. Bonfil, "The drift diffusion simulation of coupled ionic-electronic devices," M. A. Sc., Carleton University, Ottawa, Ontario, Canada, 2014.

[27] "Orgacon EL-350/175," AGFA, Septestraat 27, B-2640 Mortsel, Belgium.

[28] "DuPont 5028 silver conductor," DuPont, Research Triangle Park, NC 27709.

[29] "NOA81," Norland Products Inc., Cranbury, NJ.

[30] "NOA68," Norland Products Inc., Cranbury, NJ.

[31] K. Hyodo, "Electrochromism of conducting polymers," Electrochimica Acta, vol. 39, no. 2, pp. 265 - 272, 1994. [Online]. Available: http: //www.sciencedirect.com/science/article/pii/0013468694800626 


\section{Appendix A}

\section{SPICE Netlists for Simulations}

This appendix provides the SPICE code used in Chapters 3 and 5 simulation blocks to simulate the memristor-like devices discussed in this thesis.

EL_Node_TL

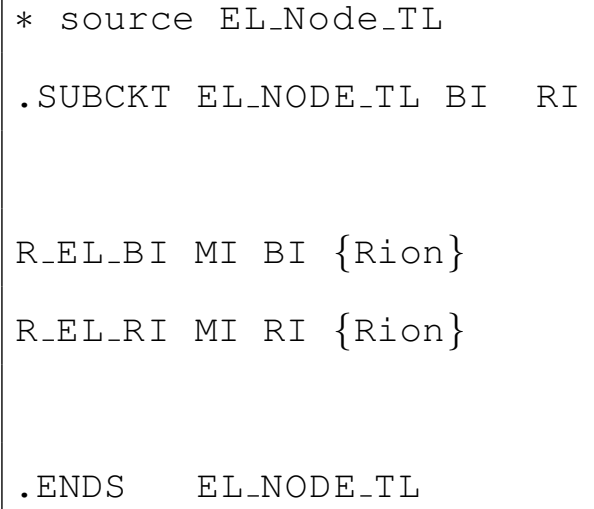


EL_Node_T

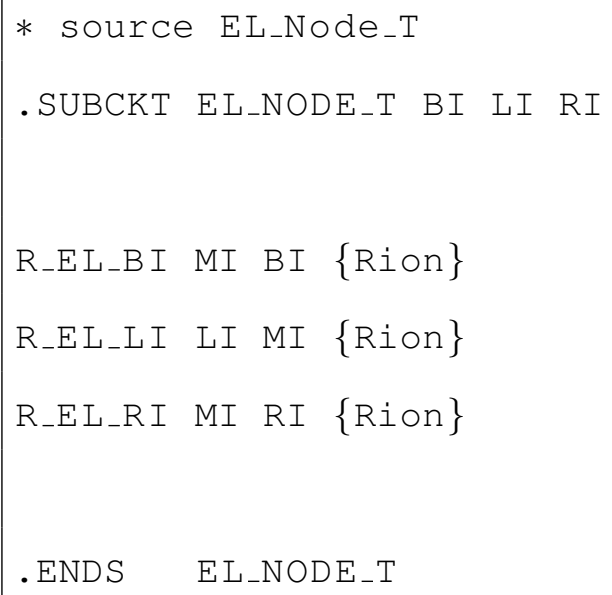

EL_Node_TR

* source EL_Node_TR

.SUBCKT EL_NODE_TR BI LI

$R_{-} \mathrm{EL}_{-} \mathrm{BI}$ MI BI $\{$ Rion

R_EL_LI LI MI $\{$ Rion $\}$

. ENDS EL_NODE_TR

EL_Node_BL

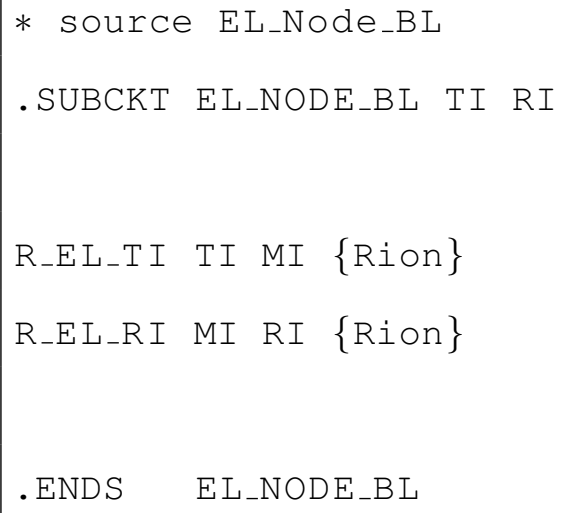


EL_Node_B

* source EL_Node_B

. SUBCKT EL_NODE_B TI LI RI

R_EL_TI TI MI $\{$ Rion $\}$

R_EL_LI LI MI $\{$ Rion

R_EL_RI MI RI $\{$ Rion $\}$

. ENDS EL_NODE_B

EL_Node_BR

* source EL_Node_BR

. SUBCKT EL_NODE_BR TI LI

R_EL_TI TI MI $\{$ Rion $\}$

R_EL_LI LI MI $\{$ Rion $\}$

. ENDS EL_NODE_BR 


\section{EC_Node_H}

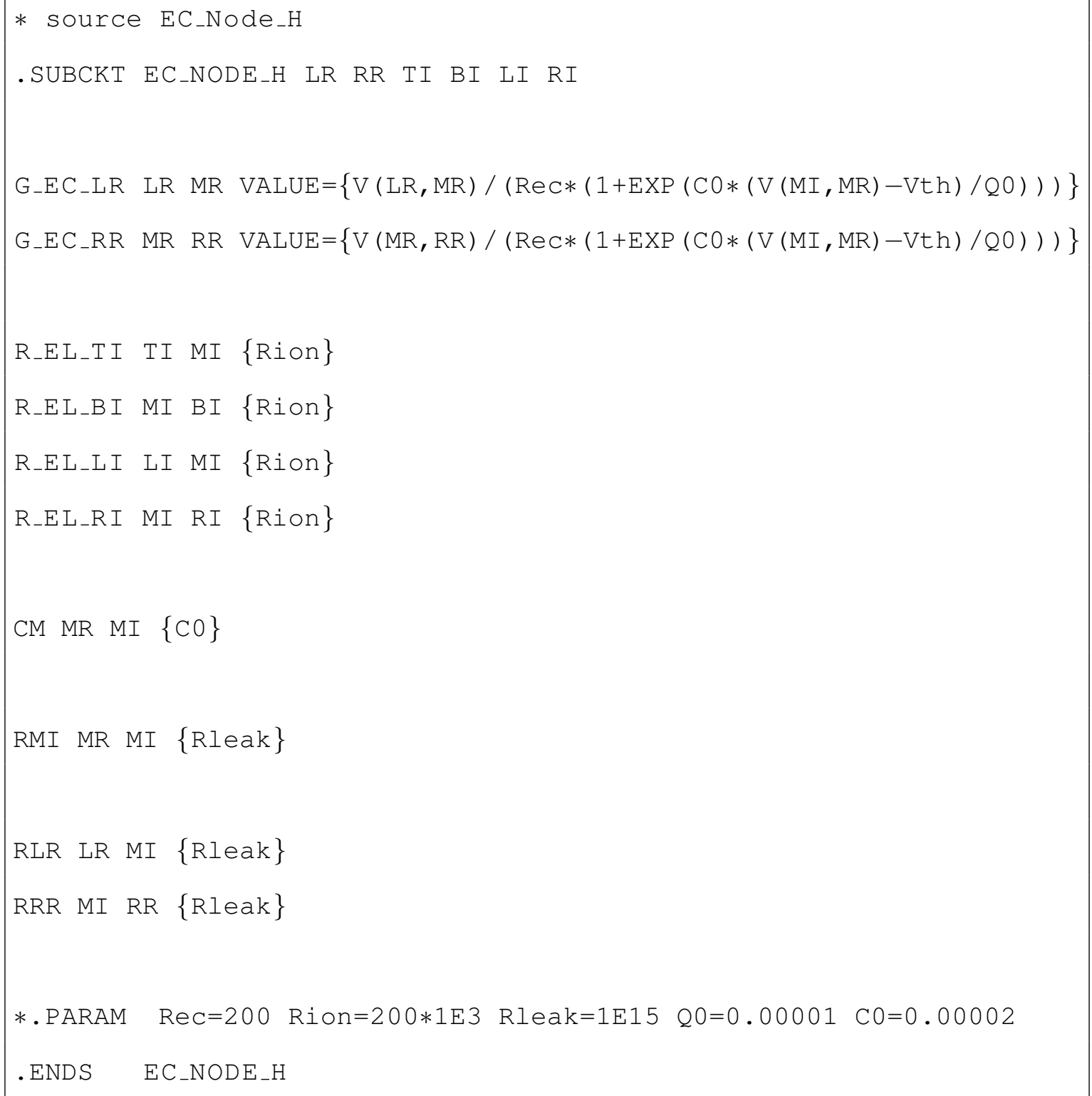

